\title{
Finding the UV-Visible Path Forward: Proceedings of the Community Workshop to Plan the Future of UV/Visible Space Astrophysics
}

\author{
A COPAG SIG Workshop held at NASA Goddard Space Flight Center, June 25-26, 2015
}

Paul A. Scowen (ASU) ${ }^{1}$, Todd Tripp (U. Mass) ${ }^{2}$, Matt Beasley (Planetary Resources, Inc.) ${ }^{3}$, David Ardila (Aerospace Corp. $)^{4}$, B-G Andersson (SOFIA Science Center) ${ }^{5}$, Jesús Maíz Apellániz (CSIC) ${ }^{6}$, Martin Barstow (U. Leicester) ${ }^{7}$, Luciana Bianchi (JHU) ${ }^{20}$, Daniela Calzetti (U. Mass) ${ }^{2}$, Mark Clampin (NASA-GSFC) ${ }^{8}$, Christopher J. Evans (ROE) ${ }^{9}$, Kevin France (U. Colorado) ${ }^{10}$, Miriam García García (IAC) ${ }^{11}$, Ana Gomez de Castro (Universidad Complutense de Madrid) ${ }^{12}$, Walt Harris (LPL) ${ }^{13}$, Patrick Hartigan (Rice U.) ${ }^{14}$,

J. Christopher Howk (U. Notre Dame) ${ }^{15}$, John Hutchings (NRC-CA) ${ }^{16}$, Juan Larruquert (CSIC) ${ }^{17}$, Charles F. Lillie (Lillie Consulting LLC) ${ }^{18}$, Gary Matthews (Harris) ${ }^{19}$, Stephan McCandliss (JHU) ${ }^{20}$, Ron Polidan (Northrup Grumman) ${ }^{21}$, Mario R. Perez (NASA HQ) ${ }^{22}$, Marc Rafelski (STScI) ${ }^{23}$, Ian U. Roederer (U. Michigan) ${ }^{24}$, Hugues Sana (AIAP) ${ }^{25}$, Wilton T. Sanders (NASA GSFC) ${ }^{8}$, David Schiminovich (Columbia U. ${ }^{26}$, Harley Thronson (NASA-GSFC) ${ }^{8}$, Jason Tumlinson (STScI) ${ }^{23}$, John Vallerga $(\mathrm{SSL})^{27}$, Aida Wofford (UNAM) ${ }^{28}$

Accepted for publication in the Publications of the Astronomical Society of the Pacific

\footnotetext{
${ }^{1}$ Arizona State University, School of Earth \& Space Exploration, PO Box 876004, Tempe, AZ 85287-6004

${ }^{2}$ University of Massachusetts, Department of Astronomy, 710 North Pleasant Street, Amherst, MA 01003-9305

${ }^{3}$ Planetary Resources, Inc., 6742 185th Ave NE, Redmond, WA 98052

${ }^{4}$ The Aerospace Corporation, 2310 E El Segundo Blvd., El Segundo, CA 90245

${ }^{5}$ SOFIA Science Center/USRA, NASA Ames Research Center, M.S. N232-12, Moffett Field, CA 94035

${ }^{6}$ Centro de Astrobiología, CSIC-INTA, ESAC campus, Camino bajo del castillo s/n, 28692 Villanueva de la Cañada, Madrid, Spain

${ }^{7}$ University of Leicester, Leicester Institute of Space \& Earth Observation, Physics Building, University Road, Leicester LE1 7RH, UK

${ }^{8}$ NASA Goddard Space Flight Center, Code 660, 8800 Greenbelt Road, Greenbelt MD 20771

${ }^{9}$ UK Astronomy Technology Centre, Royal Observatory, Blackford Hill, Edinburgh, EH9 3HJ, UK

${ }^{10}$ University of Colorado, Laboratory for Atmospheric and Space Physics, 600 UCB, Boulder, CO 80309

${ }^{11}$ Centro de Astrobiología, CSIC-INTA. Ctra. Torrejón a Ajalvir km.4, E-28850 Torrejón de Ardoz, Madrid, Spain

${ }^{12}$ Dept de Fisica de la Tierra, Astronomia y Astrofisica I, (S.D. Astronomia y Geodesia), Fac. CC Matematicas Plaza de Ciencias 3, 28040 Madrid, Spain

${ }^{13}$ University of Arizona, Lunar and Planetary Lab, 1629 University Blvd., Tucson, AZ 85721-0001

${ }^{14}$ Rice University, Dept. of Physics and Astronomy, Mail Stop 108, Houston TX 77005-1892

${ }^{15}$ University of Notre Dame, Department of Physics, Notre Dame, IN 46556

${ }^{16} 5071$ West Saanich Rd, Victoria, B.C. V9E 2E7, Canada

${ }^{17}$ Instituto de Optica. CSIC, C/ Serrano 144, 28006 Madrid (Spain)

${ }^{18} 6202$ Vista del Mar \#364, Playa del Rey, CA 90293

${ }^{19} 221$ Thomas White Blvd, Chester, MD 21619

20 The Johns Hopkins University, Dept. of Physics and Astronomy, 3400 N. Charles Street, Baltimore, Maryland 21218

${ }^{21}$ Polidan Science Systems \& Technologies, LLC, PO Box 457, Redmond, OR 97756

22 NASA Headquarters, Mail Stop 3U23, 300 E Street SW, Washington, DC 20546

${ }^{23}$ Space Telescope Science Institute (STScI), 3700 San Martin Drive, Baltimore, MD 21218

${ }^{24}$ University of Michigan, Department of Astronomy, 1085 S. University Ave., Ann Arbor, MI 48109

${ }^{25}$ Institute of Astrophysics, KU Leuven, Celestijnlaan 200D, Leuven, Belgium

${ }^{26}$ Columbia University, Dept. of Astronomy, 550 W. $120^{\text {th }}$ St., New York, NY 10027-6623

${ }^{27}$ Space Sciences Laboratory, Univ. of California, Berkeley, 7 Gauss Way, Berkeley, CA 94720-7450

${ }^{28}$ Instituto de Astronomía, UNAM, Ensenada, CP 22860, Baja California, Mexico
} 


\begin{abstract}
We present the science cases and technological discussions that came from the workshop entitled "Finding the UV-Visible Path Forward" held at NASA GSFC June 25-26, 2015. The material presented outlines the compelling science that can be enabled by a next generation space-based observatory dedicated for UV-visible science, the technologies that are available to include in that observatory design, and the range of possible alternative launch approaches that could also enable some of the science. The recommendations to the Cosmic Origins Program Analysis Group from the workshop attendees on possible future development directions are outlined.
\end{abstract}

\title{
1 Introduction
}

The Cosmic Origins Program Analysis Group (COPAG) Science Interest Group (SIG) \#2 convened a workshop $^{29}$ at NASA Goddard Space Flight Center in late June 2015 to discuss the future evolution and development of UV-Visible Astronomy within the context of what is possible technologically today and how that might enable the evolution of compelling science within the next decade. The intent of this open workshop was to bring the UV/Visible Space Astrophysics community together, and to provide input to NASA on the question of flagship missions that NASA should consider for the 2020 Decadal Survey of Astronomy and Astrophysics.

The workshop was also intended to enable the various SIG2 subcommittees to work together towards their respective goals. While the meeting was intended to be face- to-face, call-in facilities were made available and collaborative tools such as Webex and others were used to provide as creative and productive an environment as possible.

This paper is an accumulated set of reports from the speakers at the event, and the session chairs, to give a published overview of what was discussed and the conclusions that were reached during the workshop. It is intended to serve as a reference and snapshot of where the field stood as of that date, with the understanding that even today as the report is being published that many of those boundaries are being advanced as part of the Large Mission concept studies referred to in the Recommendations section at the end of the paper. This was the intended result of this workshop - to deliver a considered set of recommendations to the COPAG Executive Committee concerning strategies to advance development of space-based UV-visible astronomy for them to pass on to Dr. Paul Hertz, the Director of Astrophysics in the NASA Science Mission Directorate.

\section{The NASA Astrophysics Program - Mario R. Perez, Wilton T. Sanders}

The national investments on NASA astrophysics research, science and associated technology developments continue to be stable and significant. Available funding continues to remain either flat or slightly growing year after year in actual dollars.

The NASA astrophysics program is divided into five large programmatic themes or projects:

\footnotetext{
${ }^{29} \mathrm{http}: / /$ asd.gsfc.nasa.gov/conferences/uvvis/
} 
- Physics of the Cosmos

- Cosmic Origins

- Exoplanet Exploration

- Explorer Program

- Research and Analysis

In this presentation, we report on technologies activities both related to inception and maturation aspects, and provide an update on the Astrophysics Explorers Program.

\subsection{Strategic Astrophysics Technologies (SAT)}

In 2009, the Astrophysics Division pioneered a program for technology maturation known as the Strategic Astrophysics Technology (SAT). This technology solicitation has three components associated with each of the

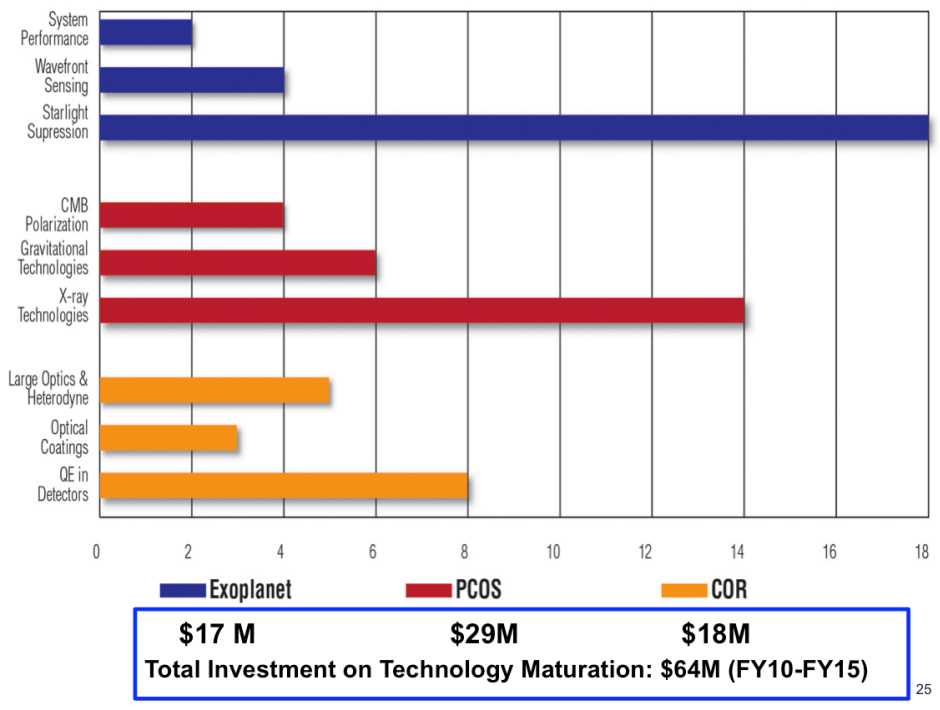

Figure 2.1 - The selected SAT investigations up to FY2015 for each of the prioritized areas within the themes are described in figure on the left. The total investments are also described for each theme. These activities have greatly advanced the state-of-the-art in some of the areas related to these applied technologies. themes, in which the Astrophysics Division is divided, namely, Physics of the Cosmos (PCOS), Cosmic Origins (COR) and Exoplanet Exploration (EXEP). This program supports the maturation of technologies of mid-range Technology Readiness Level (TRL) already developed and tested in the laboratory $(\mathrm{TRL} \leq 3)$ to a point where they can be incorporated into a flight mission with an acceptable level of risk (TRL 5 or 6). The SAT program is intended to fill the so-called "Mid-TRL Gap" of technologies that have potential but are not sufficiently mature, making them ill-suited to be part of flight programs or to be funded under basic research programs.

The funded SAT proposals are not required or expected to complete the entire development process during the period of their grants. It is expected that the proposers are able to identify verifiable milestones and provide a realistic schedule to achieve these milestones. The technologies emphasized in the SAT program are basically enabling the achievement of science drivers, as opposed to enhancing aspects of further scientific interest. The community can collaborate by identifying technology gaps, which are annually prioritized by a Technology Management Board, which is published in the Program Annual Technology Reports (PATRs). These technology gaps inform technology development needs

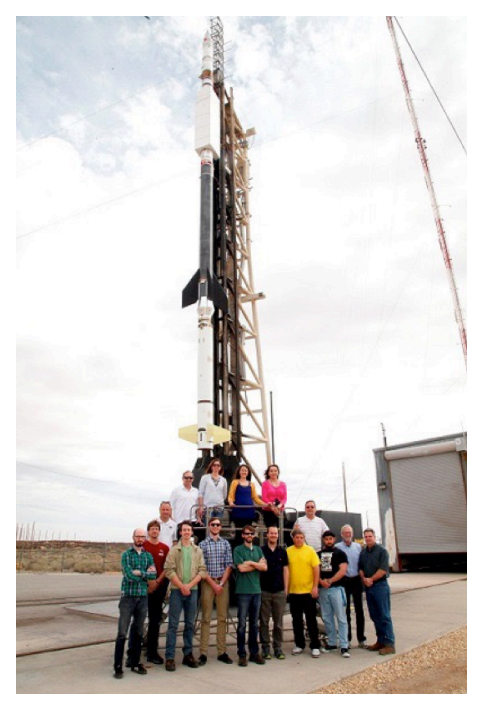

Figure 2.2 - The Colorado HighResolution Echelle Stellar Spectrograph 2 (CHESS-2) was successfully launched on 21 February 2016. The team is depicted on the left. CHESS studied translucent clouds in the interstellar medium by analyzing the UV absorption spectra of the two most abundant molecules, molecular hydrogen $\mathrm{H}_{2}$ and $\mathrm{CO}$. These data will be used to study where planets like Earth came from the by determining the raw materials available as building blocks. 


\subsection{Sub-Orbital Program}

The Astrophysics Division continues to fund several payloads, via the APRA annual solicitation, for both balloon and sounding rocket experiments. The annual number of astrophysics flights in the last five years, for each activity, has been about 3-5, throughout different seasonal campaigns. These payload flights can accomplish technology maturation (TRL by testing components in relevant environments.

\subsection{Astrophysics Explorers Program}

The goal of NASA's Explorers Program is to provide frequent flight opportunities for high quality, high value, focused astrophysics science investigations that can be accomplished under a not-to-exceed cost cap and that can be developed relatively quickly, generally in 36 months or less, and executed onorbit in less than three years.

The Explorers Program accomplishes these world-class space science investigations utilizing efficient management approaches to contain mission cost through commitment to, and control of, design, development, and operations costs. The Program also seeks to enhance public awareness of and appreciation for space science by incorporating educational and public outreach activities as integral parts of the investigations.

The Explorers Program strives to:

- Advance scientific knowledge of astrophysics processes and systems;

- Add scientific data and other knowledge-based products to data archives for all scientists to access;

- Lead to scientific progress and the publishing of results in the peer-reviewed literature to encourage, to the maximum extent possible, the fullest commercial use of the knowledge gained;

- Implement technology advancements prepared in related programs; and

- Announce scientific progress and results in popular media, scholastic curricula, and materials that can be used to inspire and motivate students to pursue careers in science, technology, engineering, and mathematics.

Though historically not always this way, the program currently administers only Principal Investigator (PI)-led science investigations for the Heliophysics and Astrophysics Divisions of NASA's Science Mission Directorate (SMD). Competitive selection ensures that the most current and best science that can be done within the cost cap will be accomplished.

Since the early 1990s, the Explorers Program has provided several classes of flight opportunities for addressing astrophysics science objectives. These mission classes are defined by their AO Cost Caps and are designed to increase the number of flight opportunities in response to recommendations from the scientific community. The Medium Explorer (MIDEX) missions solicited in 2016 have an AO Cost Cap of $\$ 250 \mathrm{M}$, roughly double the AO Cost Cap of the Small Explorer (SMEX) missions class.

Explorer Missions of Opportunity (MO) are investigations generally characterized by being part of a host space mission other than a strategic SMD mission. These partner investigations are conducted on a no-exchange-of-funds basis with the organization sponsoring the mission. Missions of Opportunity also include small complete missions and new science investigations utilizing existing spacecraft. NASA 
generally solicits proposals for MOs with each Astrophysics Explorers Program AO issued.

Current ongoing activities in the Astrophysics Explorers Program are:

- Downselection of the 2014 Astrophysics Explorers Program Concept Study Reports for both a Small Explorer (SMEX) and a Mission of Opportunity (MO) flight investigation.

- Preparing the draft (and later the final) AOs for the 2016 Astrophysics Explorers Program Medium Explorer (MIDEX) and for a Mission of Opportunity via the Second Stand Alone Missions of Opportunity Notice (SALMON-2). A Community Announcement regarding these solicitations was issued on December 16, 2015.

\section{From Cosmic Birth to Living Earth: The Future of UVOIR Space Astronomy - Jason Tumlinson}

In 2015 the Associated Universities for Research in Astronomy (AURA) published a new vision for the 2030s entitled "From Cosmic Birth to Living Earths: The Future of UVOIR Space Astronomy". The report caps a two-year AURA-chartered study of how the ambitious goals of exoplanet and cosmic origins science can be combined into a single flagship general observatory dubbed the "High-Definition Space Telescope" (HDST). Led by Co-Chairs Julianne Dalcanton (University of Washington) and Sara Seager (MIT), the study panel defines HDST as a flagship 12-m general observatory with broadband (0.1 - 3 micron) sensitivity, several novel modes of instrumentation such as high-performance optical/NIR coronography and far-UV multi-object spectroscopy, and unprecedented stability in support of high-precision astrometry, spectroscopy, and exoplanet characterization. HDST's headline science goals are to (1) detect and characterize dozens of Earth-like planets in the habitable zones of nearby stars, looking for biosignature gases in their optical/NIR spectra, and (2) to revolutionize studies of stars, galaxies, and the ultimate ingredients of life in the cosmos with high-resolution imaging and spectroscopy across the full UVOIR bandpass.

HDST builds on NASA and ESA's foundation of missions for exoplanet discovery and characterization: Kepler, JWST, TESS, and Plato have or are expected to make fundamental advances in finding and studying planets around other stars. But even if all these missions exhaust their full potential, they will not be able to find and examine the atmospheres of dozens of Earth-like planets in the habitable zones of nearby stars. JWST in particular will excel at detecting the atmospheres of planets larger than Earth transiting stars smaller than the Sun (i.e. SuperEarths orbiting M-dwarfs) where conditions are more favorable for an infrared telescope with coronagraphic contrast at only the $10^{6}$ level. Reaching the atmospheres of dozens of Earth-twin planets requires contrast ratios of order $10^{10}$, and so is beyond even the future generations of giant ground-based telescopes, which might reach a handful of Earth-size planets by surveying up to a few dozen M stars. NASA's WFIRST will advance key starlight suppression technologies and search for giant planets and SuperEarths at the $\sim 10^{9}$ level, but again will not reach dozens of Earth-twin planets. A large stride toward characterizing dozens of Earth-twin planets and searching them for biosignatures requires 10 -million-fold $\left(10^{10}\right)$ starlight suppression and collecting area large enough to obtain revealing exoplanet spectra, which in turn require a large telescope with an extremely stable wavefront and advanced starlight suppression technology (a coronagraph and/or starshade). The AURA study advocates that NASA and its community and industrial partners take steps to meet these technological challenges while the array of 
current and near-future missions lays the groundwork for HDST's search for "Living Earths".

HDST also promises revolutionary gains in capability that will make tremendous advances in our understanding of astrophysical objects and processes from the smallest stars to the most massive black holes. As a generally available flagship in the mold of the Great Observatories, HDST will unleash the widespread creativity of the astronomical community with transformative capabilities: it will have $25 \mathrm{x}$ the pixel density per area as HST in the optical, 4x better resolution than JWST in the NIR, up to 100x the point-source UV spectroscopic sensitivity, multiobject UV spectroscopy for up to 100 sources in a $\sim 3$ arcmin FOV, and extremely stable wavefronts to provide precise PSFs over long timelines. HDST's optical-band spatial resolution corresponds to $\sim 100 \mathrm{pc}$ or less physical scales at all redshifts, which will reveal the internal structure of high-z galaxies that even JWST will not resolve (Fig 1). HDST will also have the UV sensitivity to map the weak emission from metals in the circumgalactic medium of galaxies at $\mathrm{z}<2$, where gas flows drive galaxy fueling and transformation. This same UV MOS capability will enable detailed dissection of star-formation and AGN-driven feedback source-by-source in nearby galaxies. These new UVOIR capabilities promise to transform our understanding galaxies, stars, and the origins of the chemical elements.

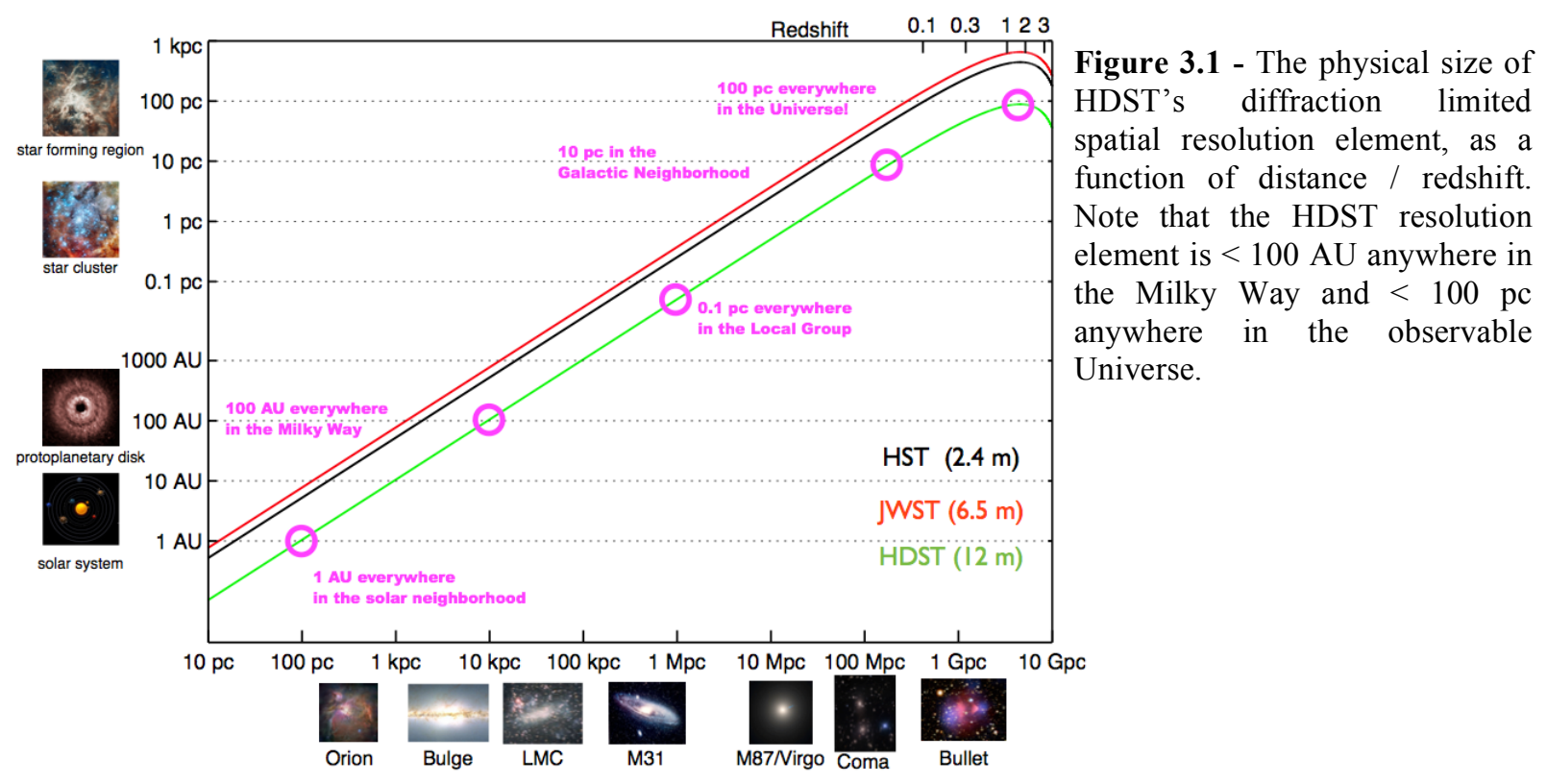

The AURA study also carefully examined the technology requirements for HDST and concluded that, with the proper investments this decade, HDST will be feasible and affordable for a new start in the next decade and launch in the 2030s. The high degree of starlight suppression required for HDST's exoplanet science is the greatest technology challenge, but new designs for coronagaphy are advancing rapidly. For cosmic origins science, the greatest technology needs are for large-format and high-QE UV and optical detectors and UV coatings that support sensitivity down to 0.1 micron while minimally affecting the optical wavefront. The report makes specific recommendations on these points that the committee hopes will feed into priorities for tech development funding in the next few years and into NASA's Science and Technology Definition Teams for flagship missions (where the HDST-like concept is known as LUVOIR). More details about HDST and contact information are available at www.hdstvision.org. 


\section{The Advanced Technology Large-Aperture Telescope (ATLAST): 2007 - 2015 - Mark Clampin / Harley Thronson}

In mid-2007, NASA issued a call for proposals for "Astrophysics Strategic Mission Concept Studies," seeking to identify concepts for scientifically compelling missions and to help identify technology developments such missions might require in advance of the 2010 NRC Decadal Survey. The Advanced Technology Large-Aperture Space Telescope (ATLAST) was one of the concepts selected to develop a technology plan to enable a large UVOIR space telescope to be considered by the NRC for development in the 2020s. The priority scientific goals of ATLAST, which would remain for its decade-long series of studies, incorporated both general astrophysics and exoplanet science on equal footing.

The ATLAST study consisted of three UVOIR telescope concepts: an $8 \mathrm{~m}$ monolithic mirror telescope and two segmented telescopes, one with a $9.2 \mathrm{~m}$ primary that could fit into an existing Evolved Expendable Launch Vehicle, and one with a $16.8 \mathrm{~m}$ primary. The $8 \mathrm{~m}$ and $16 \mathrm{~m}$ designs required a heavy lift launcher similar to the current Space Launch System. At the time, the ATLAST team considered an aperture about $8 \mathrm{~m}$ to be the minimum size needed to characterize the atmospheres of a significant number of terrestrial-mass planets in the habitable zones of their host stars, as well as providing the required spatial resolution and collecting area for other UV/optical/near-IR science goals.

In 2009, the ATLAST concept and science goals were submitted for consideration by the 2010 Decadal Survey, which responded with its highest-priority "medium" activity, investment in technologies to "[Prepare] for a planet-imaging mission beyond 2020" with mission-specific funding of $\sim 200 \mathrm{M}$ over the decade of the 2010s. This technology development program was recommended to "[Prepare] for a planet-imaging mission beyond 2020.”

In response to the NRC's recommendations, in spring 2013 NASA Goddard Space Flight Center initiated an internally funded assessment of a large-aperture UVOIR space observatory specifically intended to be sufficiently well-characterized to be considered by the 2020 Decadal Survey. This design retained the ATLAST acronym and the study was joined by JPL, the Space Telescope Science Institute, and NASA MSFC, along with regular discussions with the AURA HDST team. The ATLAST study was concluded in late 2015 with study results passed to the Large UV/Optical/IR (LUVOIR) Surveyor team.

The ATLAST scenario planning process recognized uncertainties in major science goals for this wavelength range in the $2020 \mathrm{~s}$, characteristics of plausible future launch vehicles, as well as capabilities for astronauts and robotic systems at notional operations site for an ATLAST-type mission. Moreover, at the time of the ATLAST assessment, NASA HQ had yet to prepare plans for the 2020 Decadal Survey. The final reference design was, therefore, intended to be flexible enough to be a successful candidate for future development, regardless of the scientific, programmatic, and engineering environment of the 2020s. In addition, as mandated by Congress, the ATLAST designs were all intended to be serviceable, if not actually serviced, when eventually operational.

The segmented design in one concept for ATLAST would be adaptable to different launch vehicles by increasing or decreasing the size of the segments, their number, or the adopted deployment scheme. Unfortunately, in the event with limited study resources, only a single segmented design, a $9.2 \mathrm{~m}$ configuration, was studied in depth. This aperture was validated to fit within a five-meter launch 
vehicle fairing, an industry standard, which was adopted to build confidence that the design was feasible and costs are controllable. This $9.2 \mathrm{~m}$ aperture is made up of 36 hexagonal segments and reflects the design heritage of JWST.

\section{Science}

\subsection{Workshop discussion of compelling ultraviolet-visible science drivers and facility requirements - Todd Tripp}

Context: This workshop did not occur in a vacuum; several previous (and extensive) studies had already considered the science drivers, telescope design, instrument suite, etc. for a future UV-optical telescope, and shortly before this workshop occurred, a committee commissioned by the Association of Universities for Research in Astronomy (AURA) published a detailed concept study of a $12 \mathrm{~m}$ UVvisible telescope dubbed The High Definition Space Telescope (HDST). In addition, a team at Goddard, JPL, STScI, and Marshall had spent several years studying various concepts for an $8 \mathrm{~m}$ to 16m UV-visible telescope known as The Advanced Technology Large Aperure Space Telescope (ATLAST), and a variety of materials were available from the ATLAST group. The Astro2010 Decadal Survey, while not explicitly recommending construction of a UV-optical telescope for development in the 2010-2020 period, did recognize the scientific importance of a successor to the Hubble Space Telescope and recommended funding for technology development to enable a UV-visible telescope in the 2020-2030 decade. Building on the Astro2010 report, the NASA 2013 Astrophysics Roadmap sketched a notional UV-visible mission called The Large UV-Optical-IR Surveror (LUVOIR) and provided a detailed discussion of science drivers for such a facility. Finally, a few months before this workshop, the NASA Cosmic Origins Program Analysis Group solicited white papers on large astrophysics missions to be studied by NASA in preparation for the 2020 Decadal Survey.

Science meeting goals: Given this context of rich information from very detailed existing studies, the science subcommittee identified several goals for this workshop with the philosophy that we should complement and build on previous work: (1) Are there recurring science themes in the various materials above (as well as the current research interests of the astronomical community) that point to "killer apps" for a future UV-visible-IR facility? (2) Are there important science drivers that were missed or underappreciated in previous studies? (3) What are the most important technical requirements for a future telescope? With these goals in mind, eleven speakers were invited to present science cases for a future UV-visible telescope during the science breakout session. Some speakers were selected to discuss topics where little information was available from the sources above (e.g., science drivers for ultraviolet polarimetry) while others were chosen to review hot topics that could be killer apps to motivate a future facility. One concept in the studies summarized above stands out: at this time, there is broad support for a future telescope that can search for and characterize habitable exoplanets but can also serve as a general astrophysical observatory that would be useful to a broad scientific community. Previous studies have placed significantly more emphasis on the science and technical requirements for habitable exoplanet studies, and NASA has appointed a program analysis group devoted exclusively to exoplanets, so for this workshop, we elected to focus on general astrophysics science drivers (i.e., not including exoplanets) and their technical requirements. Brief summaries of the presentations in the science breakout group are included in this paper. 
Emerging themes: After the science breakout session was completed, a townhall-style discussion occurred with all workshop attendees. This discussion briefly summarized the science breakout talks, but the emphasis was primarily on emerging themes and telescope requirements for a general astrophysics observatory. Many excellent talks were presented, and we refer the reader to the science summaries elsewhere in this paper. Speakers were asked to provide remarks about the bandpass, spectral resolution, angular resolution, field of view, multiplexing capability, etc. required by their science, and the most prominent technical requirement expressed by speakers was to have access to the far-ultraviolet band, with good sensitivity to wavelengths as short of $1000 \AA$ at least, and several speakers pointed out benefits of sensitivity all of the way to $912 \AA$. This may not be surprising - as ground-based optical telescopes grow larger and increasingly capable, the ultraviolet band naturally becomes the dominant reason to deploy a UV-visible telescope in space. The low backgrounds of space are also highly advantageous for IR astronomy as well, of course, and while some speakers commented on near-IR science, most of the talks focused on the science enabled by access to the ultraviolet. This requirement for good sensitivity in the far-UV was emphasized in the townhall discussion because good sensitivity in the far ultraviolet has been achieved in the past with various optical coatings that could be problematic for the observatory's dual purpose of studying habitable exoplanets, which can impose stringent requirements on optics in entirely different ways. Coatings that have been preferred in previous exoplanet observatory proposals/concepts (e.g., silver) could preclude ultraviolet astrophysics entirely. Therefore, it is crucial during the next few years to invest in and develop optical coatings that are suitable for both exoplanet and ultraviolet instruments. Further discussion of coatings for ultraviolet astrophysics is provided elsewhere in this paper. Another recurring theme in the science breakout talks is that future science will often require spectroscopy with good to excellent spectral resolution. Design of high-resolution spectrographs is a well-developed field, so this initially seems like a straightforward requirement, but some high-resolution spectrograph designs benefit from large-format detectors, and therefore the development of large-format ultraviolet detectors with good quantum efficiency is another area of potential investment to prepare for a future UV-visible observatory. Large-format detectors would also be highly advantageous for imaging instruments. It has been frequently noted that the UV detectors in instruments on current and past space-based telescopes have poor quantum efficiency, so development of more sensitive UV detectors is also a promising avenue for expanding the science capability of a future space telescope. Finally, there was broad consensus that a future UV-optical-IR flagship telescope must be an observatory with a suite of instruments that provides very broad capability. Given the amount of time that is required to build a flagship-class observatory, it is very difficult to predict the science questions that will be most pressing when the facility is finally deployed. For this reason, the members of this science subcommittee (and most scientists that have provided feedback in this process) broadly agree that flexibility in capabilities is crucial to enable astrophysicists of the future to fully exploit the observatory for science that we might not even imagine currently.

\subsection{UV-Optical Flagship and Massive Stars - A. Wofford, M. García García, J. Maíz Apellániz, L. Bianchi, C. Evans, H. Sana et al.}

What are massive stars? Classically, these are stars that ignite carbon non-degenerately and thus have initial masses of greater than $\sim 8 \mathrm{M}_{\odot}$. They are the progenitors of type II and Ib/c supernovae (SNe), produce black holes, and are possible progenitors of gamma-ray bursts. Although they are rare $(\sim 0.26 \%$ by number and $\sim 23 \%$ by mass assuming a Kroupa 2001 stellar initial mass function over the mass range 0.1-120 $\mathrm{M}_{\odot}$ ), and have short lifetimes (less than $\sim 50 \mathrm{Myr}$, Meynet et al. 1994), they are nonnegligible in terms of radiative, mechanical, and chemical feedback. Indeed, they are luminous $\left(10^{4}-10^{6}\right.$ 
$\mathrm{L}_{\odot}$ ), important sources of ionizing photons, inject momentum into their environment via winds and explosions, and are production sites of chemical elements (bulk of alpha-elements, some carbon and nitrogen, and some Fe-peak elements) and dust (via type II SN explosions and WC stars). Massive stars in binary systems can be strong sources of gravitational waves as demonstrated by the recent GW150914 detection (Abbot+2016).

Why are they important? There are three fundamental areas that would benefit from a deeper understanding of how massive stars work. 1) When did the first stars in the universe form and how did they influence their environments. 2) What are the cosmic origins of chemical elements, in particular those essential to life as we know it. 3) How exchanges of mass and momentum between massive stars and the environment shape the evolution of galaxies. In particular, understanding massive stars is crucial for developing the spectral population synthesis models that are used for interpreting observations of unresolved massive-star populations and star-forming galaxies across redshift.

What is the problem? The designs of past and current UV satellites are not optimized to observe i) large samples of massive stars or ii) stars beyond the nearest Local Group galaxies. Large samples are needed in order to separate the many competing factors that influence the observed properties of massive stars (mass, chemical composition, convection, mass-loss, rotation, close-binarity, magnetic fields, etc.), and which affect their nuclear reaction rates, the duration of their evolutionary stages, and their final stages. Massive-star evolution is particularly uncertain at initial stellar masses greater than 20 Msun, where evolutionary stages and supernova explosions are not well mapped. Large samples are also needed to obtain extragalactic UV extinction curves along many more sightlines. After 25 years of HST, the number of extragalactic UV extinction curves is extremely low (a few sightlines in LMC, $\mathrm{SMC}, \mathrm{M} 31$ ). The mean UV extinction law for the SMC which is usually taken as template for low-Z galaxies is based on a handfull of stars with diverse extinctions (with some showing the dust bump and some not; Maíz-Apellániz \& Rubio 2012). Our scarce knowledge of UV extinction translates into large systematic uncertainties in stellar masses and star formation rates of galaxies (as large as one order of magnitude for the SFR, Bianchi+11). Observations beyond the Local Group are necessary in order to sample regimes of extreme low metallicity and star-formation, that build the bridge to higher redshift results. In particular, regions with metallicities well below 1/7 solar and approaching primeval conditions, and star clusters of initial mass greater than $\sim 10^{5} \mathrm{M}_{\odot}$, as during the cosmic peak of star formation. The dependency of stellar parameters with metallicity has hardly been addressed in part because we can't study individual stars in galaxies beyond $1 \mathrm{Mpc}$. The few studies in 1/7 solar metallicity dwarf irregulars, including IC1613, are controversial (Tramper+11, García+14; Bouret+15). The two nearest most metal-poor (1/30 solar) star-forming galaxies in the nearby universe are DDO $068(7.9 \mathrm{Mpc})$ and I Zw 18 (18 Mpc), and a pair of galaxies like the Antennae ( 20 Mpc) host young massive clusters of $\sim 10^{5} \mathrm{M}_{\odot}$ and greater (Whitmore+). Progress could be achieved by observing these targets.

What is needed? UV and optical spectroscopy are how main photospheric and wind parameters of massive stars are obtained. To characterize winds we need UV spectroscopy, from 900-1800 $\AA$ at $\mathbf{R}=\mathbf{1 0 , 0 0 0}$ (although 5,000) is probably enough. The UV resonance lines of OVI, SIV, PV, SiIV, CIV, $\mathrm{NV}$ and non-resonance CIII, OIV, OV, NIV constrain shocks in the wind, micro-clumping, macroclumping and mass-loss, and provide in most cases the only diagnostic for terminal velocity. Optical spectroscopy, containing $\mathrm{H} \alpha$ and HeII4686, at $\mathbf{R}=\mathbf{1 0 , 0 0 0}$. If we want to explore Local Group galaxies (beyond the Magellanic Clouds) and redenned regions of the Milky Way, we would need to reach in the UV $1 \mathrm{E}-17 \mathrm{erg} / \mathrm{cm}^{2} / \mathrm{s} / \AA$ in $2 \mathrm{~h}$ exposure time, with $\mathrm{SNR}=20$ per resolution element at least. 
To determine stellar properties (temperature and gravity), $\mathrm{R}=5,000$ in the optical range (3500-7000 $\AA$ ) is enough. However, $\mathbf{R}=\mathbf{2 0 , 0 0 0}$ would enable us to get: chemical abundances, projected rotational velocities, macroturbulent broadening. The latter three points are currently being investigated as indirect probes of the internal processes occurring during evolution. In particular, macroturbulence may be connected to stellar pulsations and/or turbulent pressure generated by convection zones. In this case we would need $\mathrm{R}=20,000$ with $\mathrm{SNR}=100$. If we want to comfortably explore Local Group galaxies, we need to reach $\mathrm{V}=20.5$. With regards to extinction by dust, detailed maps in the Magellanic Clouds and beyond could be achieved with a multi-object FUV plus optical spectrograph with high sensitivity, via panchromatic photometry of resolved stars or low resolution spectra. We would certainly benefit from any multiplexing capability within 30" (projected size of a Local Group OB association) to 10' (rough diameter of a LG dwarf irregular galaxy), and sub-arsecond spatial resolution.

What we would get? Complete color-magnitude diagrams of massive-star populations down to $1 \mathrm{M}_{\odot}$ for testing stellar evolution tracks over a wide range of metallicities and star formation history scenarios. Simultaneous medium-resolution co-spatial UV+optical spectroscopy for $\sim 100$ massive stars in one exposure in Local Group galaxies for separating the astrophysics that determine the properties of massive stars and determining chemical abundances. Multiple exposures to improve S/N, address stellar variability, and monitor candidates for SN explosions and gamma-ray burst. Detailed dust extinction maps in the Magellanic Clouds and beyond. Large samples of chemically enriched wind bubbles around evolved massive stars and SNRs for constraining stellar nucleosynthesis and galaxy enrichment. Finally, emission and absorption spectra of the ISM in galaxies covering a wide range of conditions.

\subsection{Nearby Galaxies Under Magnification: New Science with a Future UVOIR Telescope - Daniela Calzetti}

Nearby galaxies provide unique test-beds for theories of star formation and galaxy evolution, because they can be dissected into elemental components: stars, star clusters, gas clouds, etc. which can be investigated and compared against models with exquisite detail.

The Hubble Space Telescope has produced a revolution in this field, by enabling the derivation of the histories and properties of both young and old stellar populations and the gas they ionize in galaxies up to a few Mpc. The combination of the James Webb Space Telescope and WFIRST will expand this legacy by pushing the investigation of the nearby Universe into new territory. For instance, we will be able to unravel the physical foundation of the star formation-gas relation (the Schmidt-Kennicutt Law) and the spatially-resolved star formation histories of galaxies over the $200 \mathrm{Myr}-10 \mathrm{Gyr}$ range out to about 6-8 Mpc. The low-end of the stellar Initial Mass Function and its variations will be investigated out to $\sim 0.5 \mathrm{Mpc}$. We will be able to access locales where to study the physics of the dust processing and formation out to $\sim 10 \mathrm{Mpc}$. Although the limits in accessible cosmic volume are somewhat fluid, as they depend on the diagnostic used and crowding of the systems under investigation, a distance limit of 6-8 Mpc secures mostly dwarf galaxies, since only about a dozen giant spirals and at most one giant elliptical are present in this volume. Furthermore, the JWST and WFIRST are optimized for the near/mid-infrared regime, while young stellar populations in the age range $2-100$ Myr emit the bulk of their energy in the UV. Volume and wavelength constraints strongly limit the range of accessible environments by existing and planned space facilities. These are the environments where complex physical processes can be studied and models to describe them tested. 
A UV-optical optimized telescope with an aperture $\geq 5$ times that of the HST, will detect and resolve individual massive stars $\left(\geq 10 \mathrm{M}_{\mathrm{o}}\right)$ and star clusters as low in mass as a few $1,000 \mathrm{M}_{\odot}$ and as old as $\sim 100-200$ Myr out to $\sim 100 \mathrm{Mpc}$. Star formation histories as far back as $\sim 10$ Gyr will be obtained out to $\sim 20 \mathrm{Mpc}$, i.e., out to and beyond the Virgo Cluster, with the same quality achievable today out to $\sim 3-4$ Mpc with the HST. The local $\sim 100 \mathrm{Mpc}$ volume contains several (Ultra) Luminous Infrared Galaxies (U/LIRGs). U/LIRGs are common denizens of the high-redshift Universe, becoming as common as normal star-forming galaxies, such as the Milky Way, above z 1 (Murphy et al. 2011).

At least three open problems will be addressed by such capability: (1) determine the upper end (slope, maximum stellar mass) of the stellar Initial Mass Function and establish any variation; (2) establish the modes of galaxy growth; and (3) reconcile the Local and Cosmic star formation histories. In order to answer all three questions, the UV/optical and large-aperture capabilities need to be coupled with large detector's Field-of-View (FoV, about 3'x3', in order to sample a sizeable portion of each galaxy) and, for the IMF, with multi-object (UV/optical) spectroscopy.

Any quantity related to star formation rates (SFRs), short-timescale $(<300-500$ Myr) star-formation histories, feedback, or chemical enrichment of galaxies, will involve the high-end of the IMF. This is because stars above a few solar masses emit, via stellar winds and supernovae explosions, virtually $100 \%$ of the ionizing photons, over $80 \%$ of the non-ionizing UV photons, and provide the vast majority of the energy, momentum, and chemical output from stars. Variations at the high end of the IMF can thus have a major influence on the interpretation of galaxy evolution. For instance, SFR measurements can be over- or under-estimated by as much as a factor $\sim 2$, depending on the strength of the IMF variation and the range of stellar masses considered. This impact can, additionally, be non-uniform across galaxy populations and across time.

Models can support both universal and variable IMFs, owing mainly to the complex physics and the variety of conditions under which stars form in gas clouds. Direct and indirect measurements of the shape of the IMF in a range of galactic environments are the only tool available to constrain potential variations. One example of indirect measurement of the high-end of the IMF involves probing the aggregate properties of the young star cluster populations in nearby galaxies (Calzetti et al. 2010; Andrews et al. 2013; Andrews et al. 2014). A stringent test for IMF variations requires covering a wide range of galaxy morphologies, masses, SFRs, specific SFRs, and external environments, including interacting and merging systems. This requirement is satisfied by the $\sim 100 \mathrm{Mpc}$ local cosmic volume probed by the large-aperture UV/optical telescope.

Models of galaxy growth predict that both minor mergers and inside-out star formation should contribute to the formation of the bodies of galaxies; however, the relative importance of each mechanism is still under debate (Brown et al. 2008, Wang et al. 2011). The outskirts of galaxies are dynamically 'quiet' environments, where the imprint of past events and structures persists for multiple Gyrs. These are perfect settings for testing models of galaxy growth, as minor mergers and inside-out star formation predict different population mixes. They are also excellent locales for investigating modes of star formation, owing to their extreme conditions of density, pressure, metal enrichment, dust content, and response to mechanical feedback. Given the characteristics of the environments, UVoptical capabilities need to be coupled with a large FoV (see above), and the sensitivity afforded by a large-aperture telescope, in order to acquire statistically significant numbers of outer disk stars and optimally complement the near-infrared surveys that will be performed with WFIRST. 
The size of the Local Universe that is representative of the cosmic Volume, i.e. the local volume that yields the same average properties as the evolving Universe, is not a settled number. The Universe within the local 3-4 Mpc is over-dense relative to the cosmic average, and yields SFR densities that are about a factor $\sim 2$ higher than the cosmic value over the past 4-6 Gyr (i.e., since z 0.4-0.7, Drozdovsky et al. 2008; Williams et al. 2011). The number of Core Collapse Supernovae within the local $11 \mathrm{Mpc}$ is higher by about a factor 1.7-2 than the number that would be predicted from the UV emission of all galaxies within the same volume, and it is even higher than the number predicted from the H $\alpha$ emission (Botticella et al. 2012; Horiuchi et al. 2013). No such discrepancy is observed on cosmic scales for $0<\mathrm{z}<1$ (Dahlen et al. 2012). Solving these discrepancies and determining the Representative Local Volume will be the task of the next large-aperture UV/optical facility.

\title{
5.4 Some Science Cases for Space UV Polarimetry in the $21^{\text {st }}$ Century - B-G Andersson
}

\author{
"Polarization is the crucial third leg of the astronomer's light-analysis tripod" - D.P. Clemens
}

Polarization provides a powerful, but all-too-often overlooked, tool of observational astronomy that allows us to probe magnetic fields in stars and the interstellar medium, reveals structures in unresolved, asymmetric objects, such as circumstellar disks, and enables the study of obscured or below the diffraction limit phenomena, including in the inner parts of Active Galactic Nuclei. Broad wavelength coverage, including the ultraviolet, provides critical constraints on many astrophysical processes. While many applications for the ultraviolet part of the spectrum can already be identified, UV polarimetry is poorly explored and provides significant discovery space. Because polarimetry is inherently photonintensive, large apertures are needed for efficient observations. However, the Stokes-vector $(\mathrm{Q}, \mathrm{U}, \mathrm{V})$ decomposition required for polarimetric analysis can be combined to yield the intensity (Stokes I) needed for photometry or spectroscopy, and hence no information is lost in polarimetry mode and the polarization optics transmission is typically very high. While some telescope designs can preclude the efficient acquisition of polarimetric data, careful considerations in the mission design can preserve the ability to include polarimetry in the science program for future (space-based) observatories. Below I outline a small number of concrete examples of science enabled by space-based UV/Optical polarimetry.

\subsubsection{Interstellar Medium Studies}

Dust-induced interstellar polarization allows studies of the interstellar magnetic field geometry and strength. A quantitative, empirically well-tested, theory of grain alignment is now available, allowing reliable quantification of the magnetic field, of the dust and other environmental parameters (Andersson, Lazarian \& Vaillancourt, 2015). Radiative Alignment Torque (RAT) theory predicts that paramagnetic grains are aligned if exposed to a radiation field with wavelengths less than the grain diameter. This e.g. directly explains the shape of the UV-NIR polarization curve which requires that for most diffuse ISM line of sight - grains smaller than $\sim 0.045 \mu \mathrm{m}$ are unaligned. However, for some of the lines of sight probed in UV polarimetry with WUPPE or HST/FOS (e.g. Martin, Clayton \& Wolff, 1999), significant UV polarization is seen, indicating alignment of grains down to $\sim 0.01 \mu \mathrm{m}$. Under RAT alignment this can be understood if some of the dust is exposed to radiation shortward of $912 \AA$, providing a probe of the Galactic hard-UV radiation field. For two of the lines of sight with enhanced UV polarization, polarization in the $2175 \AA$ extinction "bump" is also seen. Further UV polarimetry can therefore address the nature of the carrier of this feature, allowing a better understanding of the extinction and mineralogy of the dust. An alternative interpretation of the 
enhanced UV polarization proposes that these lines of sight represent local enhancements in the strength of the ISM magnetic field. Under such conditions, very small grains can be efficiently aligned by paramagnetic relaxation alignment, and would allow a direct measurement of the magnetic field strength (Hoang, Lazarian \& Martin, 2014). For atoms and ions with [hyper-] fine structure transitions, an effect similar to RAT alignment leads to line polarization, which can be used to probe the magnetic field geometry and strength. Many promising transitions occur at UV wavelengths (Yan \& Lazarian, 2008)

\subsubsection{Unresolved Asymmetric Objects}

Polarization allows structures to be derived in unresolved asymmetric sources, by interpreting the scattered light or Zeeman effect lines. Because of the differing spectral dependence of electron (Thompson) scattering, and scattering by polarizable molecules or dust in the small particle limit (Rayleigh scattering), a significant amount of physical information can be gleaned from multiwavelength polarimetry. For sources with rotational or orbital velocities, time-resolved polarimetry can be used to construct 3D models of structures and magnetic fields (e.g. Morin et al., 2008).

UV spectropolarimetry provides a probe of the physical state of circumstellar matter, unresolved by other techniques, for many stellar environments. Spectral-type Be stars are rapidly rotating massive stars that are laboratories for understanding the physics of hot circumstellar gas disks. The polarization from Be star disks can be used to derive disk temperature, density, and structure, using models of electron scattering modulated by line-blanketed depolarization (Bjorkman, Bjorkman \& Wood, 2000).

Most massive stars occur in binary systems, influencing their evolution and possible end-states as supernovae or gamma-ray bursters. UV polarimetry of the wind collision regions of high-mass binaries allow detailed characterization of shock structures and provide constraints on the effects of binarity in massive stellar evolution (Lomax et al. 2015). Magnetized evolved binaries are likely precursors to SN Ia explosions. Polarimetry of Zeeman and Cyclotron lines from these objects provides unique information on the magnetic field characteristics in these systems, and its influence on their evolution. Stokes spectroscopy of the Cyclotron features allow full physical analysis, including temperature, density, accretion rate and white dwarf mass, as well as magnetic field strengths (Thomas et al., 2012).

While still somewhat controversial, the use of Rayleigh scattering from the extended atmospheres of exo-planets may provide a novel way to characterize their atmosphere and a unique way to establish the orientation of the orbital plane. Because of the $\lambda^{-4}$ dependence of Rayleigh scattering, sensitive, time resolved, UV polarimetry is needed to maximize the contrast at the low polarization levels expected and required to resolve the conflicting results to date (Berdyugina et al. 2011, Wiktorowicz, 2009)

\subsubsection{Revealing Obscured Sources}

As scattering redirects radiation, sources whose direct line of sight is obscured can be revealed in polarized light, differentiating between the scattered light and general diffuse emission. This can occur both in circumstellar environments and in active galactic nuclei (AGN). Polarized light from AGN originates as synchrotron emission from jets extending up to several kpc from the nucleus, and is due to scattering by dust or electrons in the vicinity of the AGN, obscured in direct light by the torus (Packham et al. 2011). The accretion disk emission in AGN peaks in the UV, and therefore polarimetry at these wavelengths is crucial to elucidate these sources. The scattering is by small particles yielding $\mathrm{p} \sim \lambda^{-4}$. Therefore, the polarized component is more easily distinguished from other emission in the UV region. This enhances the contrast between AGN structures, such as scattering cones, and the unpolarized light from surrounding regions in imaging polarimetry (Kishimoto, 1999), affording key 
spatial information as to the true location of the AGN. The polarized spectrum from AGNs elucidates the nature and opacity of the scatterers and the dynamics of the circumnuclear disk. The large distances and photon needs of UV polarimetry make AGN observations stretch the capability even of HST. UV polarimetry with large UV space-based telescopes will allow studies of the 3D structure of the AGN central engines, accretion disks and surrounding dusty tori, and thereby the evolution of AGN.

\subsubsection{Summary}

A significant discovery space exists for UV polarimetry with large apertures, providing high total photon counts, particularly as part of a broad wavelength coverage. In addition to the examples given here, improvement in theory now guide observations and interpretation to expand the areas of research. Care must, however, be exercised in optical designs to not preclude the inclusion of polarimetry.

\subsection{Opportunities for Star and Planet Formation Research with a Large-Aperture Optical/UV Telescope - P. Hartigan}

Understanding how stars and planets form is a large and growing area of current astronomical research. For the latest HST proposal round (Cycle 22) if we count the panels of Extra-Solar Planets, Debris Disks, Resolved Star Formation, and half of Cool Stars and ISM/Circumstellar Matter as related to this topic, the subject area constitutes a bit more than $1 / 6$ of all the submitted proposals, all in the optical/UV. Research related to extragalactic star formation commands a similarly high fraction. At mm-wavelengths the fraction of star-formation research is even higher, with the panels of ISM/Star Formation/Astrochemistry and Circumstellar Disks/Exoplanets/Solar System accounting for nearly half of the approved proposals for ALMA in Cycle 2. A space-based large-aperture optical/UV telescope would lead the research efforts in star formation in many ways. Figure 5.5.1 summarizes the principal physical processes associated with circumstellar disks and outflows. Understanding these phenomena constitutes much of the current research in this discipline. While we must always consider limitations imposed by dust extinction when assessing any specific star-formation proposal, this figure illustrates why the optical/UV spectral region is essential for investigating how stars and planets form.

Examples where optical/UV observations play key roles include:

- Physics of Accretion Columns: Stars accrete as much as $10 \%$ of their final masses from circumstellar disks, and this process affects the masses, coalescence, migration, and composition of protoplanets as they form. Through a process that is poorly-understood, material in the disk is lifted onto the stellar magnetic field and funneled to the stellar photosphere, where it encounters a strong accretion shock. Radiation from this accretion hot spot emits primarily in the UV, and high-spectral resolution of emission line profiles in the optical and UV show both inverse P-Cygni profiles indicative of infall, and normal P-Cygni profiles that result from outflow. A large UV/optical telescope would enable direct observations of this process for thousands of stars, and we may even be able to observe the effects of accretion onto protoplanets in the most nearby systems either with spatially-resolved imaging, or by observing periodic velocity signatures in the emission lines.

- Chromospheric Activity and Young Stellar Winds: Low-mass stars like the Sun are born with active photospheres and exhibit strong X-ray fluxes, chromospheric emission lines and starspots. Radiation from coronal and chromospheric activity and stellar winds strongly 
influence how atmospheres evolve around planets as they form. A UV/optical telescope that operates in the FUV between $10.2 \mathrm{eV}$ and $13.6 \mathrm{eV}$ is the only way to trace ionized species such as $\mathrm{O} I \mathrm{~V}$, which bridge the gap between optical-emitting plasmas at $\sim 10^{4} \mathrm{~K}$ and X-ray emitting plasmas at $10^{6} \mathrm{~K}$. Monitoring the UV emission lines over time will provide important constraints on flare activity and stellar cycles at the earliest ages.

- Jet Formation and Collimation: We know that accretion drives outflow, but the mechanism by which disks redirect infalling material from a disk into a collimated supersonic flow lies hidden within $\sim 10$ AU of the star, just below current angular resolution limits for the closest systems. The phenomena of accretion disks and outflows is universal throughout astrophysics, and is easiest to observe in young stars where the densities are high enough for the flows to radiate emission lines when they pass through shock fronts. In addition, jets from young stars are fast enough to facilitate observable Doppler motions with moderate spectral resolution and to exhibit detectable proper motions over a few years for the closest systems. Issues related to magnetic reconnection of tangled kink-unstable toroidal fields, detecting rotation in jets, learning what causes jets to precess, and understanding why jets eject pulsed flows will only be within reach using a facility like the proposed UV/optical telescope.

- Physical Conditions within Jet Shocks: We have learned a great deal about shock waves in jets in the last decade thanks to the stability and spatially-resolved spectra achievable with HST. The field of ISM plasma diagnostics is over a half-century old, and the same techniques are used to infer electron densities, temperatures, and ionization fractions within each pixel in young stellar jets. The physics of L-S (spin orbit or Russell-Saunders) coupling implies that most of the energy levels of common ions have energy levels of a few eV. For that reason, the best diagnostics of density and temperature are in the optical/UV. Measurements of these line ratios are the best way to compare with the output from modern 3D shock codes, and new theoretical shock models that optical emission line ratios can also be used to infer magnetic field strengths in flows as long as the observations have sufficient spatial resolution $(0.2 \operatorname{arcsec}$ for the closest systems) to separate collisionally-excited Balmer emission from the shock from forbidden-line radiation in the cooling zones. Emission line studies of this kind also determine abundance depletions, which clarify the initial conditions present when the star formed as well as constrain dust formation and destruction in disks. While HST has showed the way for this type of research, currently only a handful of jets are close enough and bright enough to resolve the cooling zones of jets and create detailed movies of the MHD evolution of shocked flows.

- Dynamics of Gas Disks: Thermal radiation from dust is the domain of IR and mm-wave observations. Scattering from dust in the optical/UV provides the only means to detect small grains present in the infalling envelope or that become entrained in the outflows. As heavier dust settles to the midplane of the disk, a dust 'wall' should form within a few tenths of an AU from the star where dust evaporates into gas. It is in the gas where most of the mass resides, and we must follow this component if we are to learn how gas-giant planets and their reservoirs evolve. The principal tool used to study $\mathrm{H}_{2}$ in disks is with fluoresced UV emission and absorption lines. These mainly lie in the Lyman and Werner bands between $10-13 \mathrm{eV}$. When these lines are resolved spectrally, they determine whether or not a slow, possibly photoevaporative wind emanates from the disk (e.g. Figure 5.5.1). Such studies have only been done to date for the closest gas disks. A large-aperture UV/optical telescope should be able to resolve the region where photoevaporative flow is launched, and provide an actual image of the gas in another solar system on inner planet scales.

- Binaries: Roughly half of the stellar systems in our galaxy are binaries. Studying binary 
fractions at close separations at the earliest ages is the only way to learn how binary separations, and thereby the angular momentum distribution of the systems, evolve with time. At large separations each stellar component can have its own disk, but these become truncated once the separations become on the order of the disk sizes. Numerical models have shown that eccentric systems can force pulses of accretion at periastron, and these close passages may also generate spiral waves and disk warps. The structure of binary jets is completely unknown on small scales. Research into these areas requires high-contrast imaging at the finest spatial scales possible, and is ideal for an optical/UV space-based telescope. There is even the possibility of using eclipse-mapping techniques once enough close binaries are found.

Figure 5.5.2 summarizes the impact that a 10-m class optical/UV telescope would have upon spatiallyresolved studies of star and planet formation. The colors in Figure 5.5.2 correspond to the various scales depicted in Figure 5.5.1. For example, in the optical at the distance to Taurus/Auriga, HST currently resolves scales of $\sim 5 \mathrm{AU}$, at the boundary between the envelope and disk scales depicted in Figure 5.5.1. A 10-m class telescope would push this boundary all the way out to Carina, where the number of objects increases from $\sim 100$ to over 30000, enabling statistically-significant studies of star formation phenomena. Pointing a 10-m optical/UV telescope at a disk in Taurus would resolve Earthlike orbital distances of $\sim 1$ AU. Note that the disk/jet emission of interest on these spatial scales will be located close to a very bright point source, a difficult task for any future ground-based AO system. The potential for new discoveries related to a particular object grows as the distance to the object decreases, both from the standpoint of improved spatial resolution and from being able to observe lower flux levels with better signal-to-noise ratios. Most stars form in regions that contain massive stars, so to understand star formation on a global scale we must extend our investigations to these types of regions, which, as shown in Figure 5.5.2, are more distant than their lower mass counterparts. Young stellar disks and planetary systems born in the vicinity of massive stars will evolve quite differently from those that develop in regions that are largely shielded from external radiation. Regions with massive stars emit prodigious amounts of UV radiation, create cavities and H II regions in their nascent molecular clouds, and drive powerful winds. All of these processes affect the amount of mass that is available to form disks and planets around the lower mass stars. The shape of the IMF implies that all high-mass star-forming regions produce large amounts of low-mass stars as well. Hence, statistical studies are best done in such regions. The takeaways here are (i) currently the best spatial resolution in the optical/UV stops $\sim 5-10$ AU for the nearest disks, just where planetary orbital phenomena become manifest: a large-aperture optical/UV telescope will bring the blurred, inaccessible inner planet regions of disks into focus for the first time; and (ii) new phenomena observed in the last decade at the limit of HST's capabilities have transformed how we draw the star-formation scenario in Figure 5.5.1: the proposed telescope will extend studies of these phenomena from a handful of systems to thousands, and enable studies of entire massive star formation regions that have to date only been possible for isolated, low-mass regions.

On the spectral frontier, higher spectral resolutions are required to probe rotational broadening at larger distances from the star (Figure 5.5.3). In this sense, spectroscopy and imaging are complimentary - it is more difficult to image close to the source, but easier to resolve orbital motions there. A spectrometer with a spectral resolution of $\sim 10^{5}$ will resolve thermal widths for all gas in the warm disk. Lower spectral-resolution suffices for gas in H II regions or behind strong shocks. Having a slit-spectrograph is crucial - most projects will want to observe how plasma processes vary across the disk and along flows. 


\section{Star Formation Overview}

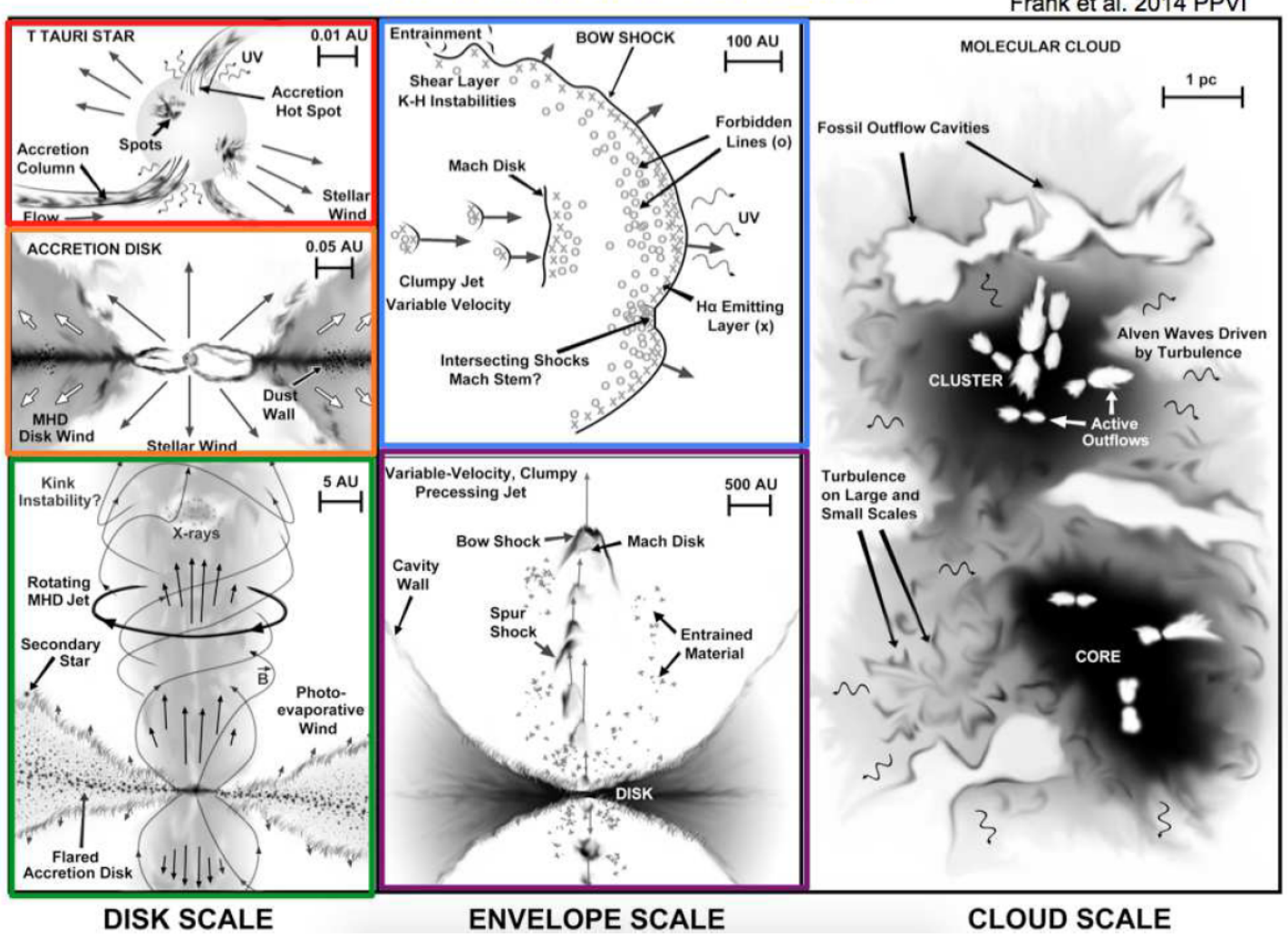

Figure 5.5.1 - Star formation summary, adapted from Frank et al. (2014, Protostars \& Planets VI). The drawing is to scale, and summarizes the main physical processes associated with accretion disks and collimated jets. The colors that outline each panel are also used in Figures 5.5.2 and 5.5.3.

\section{Spatial Scales}

\begin{tabular}{|c|c|c|c|c|c|}
\hline \multirow[t]{2}{*}{$\begin{array}{c}\text { Increasing Mass/ } \\
\text { of Region }\end{array}$} & Size $\longrightarrow$ & & \multicolumn{3}{|c|}{ Statistical Studies Possible } \\
\hline & Gas Disk & $\begin{array}{l}\text { loud with Dusty } \\
\text { Accretion Disks }\end{array}$ & $\begin{array}{r}\text { Cluster with a } \\
\text { few O stars }\end{array}$ & $\begin{array}{l}\text { Cluster with }>\sim 100 \\
\text { s of O stars }\end{array}$ & $\begin{array}{l}\text { Starburst } \\
\text { Cluster }\end{array}$ \\
\hline $\begin{array}{l}\text { Nearest Example: } \\
\text { Distance: } \\
\text { \# of Stars in Group: }\end{array}$ & $\begin{array}{l}\text { TW Hya } \\
54 \mathrm{pc} \\
\text { Isolated }\end{array}$ & $\begin{array}{c}\text { Taurus/Auriga } \\
140 \mathrm{pc} \\
50\end{array}$ & $\begin{array}{l}\text { Orion } \\
410 \mathrm{pc} \\
1000\end{array}$ & $\begin{array}{l}\text { Carina } \\
2.3 \mathrm{kpc} \\
30000\end{array}$ & $\begin{array}{l}0 \text { Doradus (LMC) } \\
49 \mathrm{kpc} \\
>30000\end{array}$ \\
\hline $\begin{array}{l}\quad 1.2 \lambda / D \\
\text { HST UV (2000 A): } \\
\text { HST Optical }(5000 \text { A): }\end{array}$ & $\begin{array}{l}1.1 \mathrm{AU} \\
2.8 \mathrm{AU}\end{array}$ & $\begin{array}{l}2.9 \mathrm{AU} \\
7.2 \mathrm{AU}\end{array}$ & $\begin{array}{l}8.5 \mathrm{AU} \\
21 \mathrm{AU}\end{array}$ & $\begin{array}{l}50 \mathrm{AU} \\
120 \mathrm{AU}\end{array}$ & $\begin{array}{l}1000 \mathrm{AU} \\
2500 \mathrm{AU}\end{array}$ \\
\hline $\begin{array}{c}1.2 \text { A / D } \\
12-m \text { UV (2000 A): } \\
12-m \text { Optical (5000 A) }\end{array}$ & $\begin{array}{l}0.22 \mathrm{AU} \\
0.56 \mathrm{AU}\end{array}$ & $\begin{array}{r}0.58 \mathrm{AU} \\
1.4 \mathrm{AU}\end{array}$ & $\begin{array}{l}1.7 \mathrm{AU} \\
4.2 \mathrm{AU}\end{array}$ & $\begin{array}{l}9.5 \mathrm{AU} \\
24 \mathrm{AU}\end{array}$ & $\begin{array}{l}200 \mathrm{AU} \\
500 \mathrm{AU}\end{array}$ \\
\hline $\begin{array}{l}\text { Consideration } \\
\text { - What emits/absorb } \\
\text { - Extinction } \\
\text { - Spectral informatio } \\
\text { important than spa } \\
\text { especially in red ar }\end{array}$ & $\begin{array}{l}\text { S } \\
\text { S in UV/opt } \\
\text { tial resolution } \\
\text { d orange zones }\end{array}$ & $\begin{array}{l}\text { UV } \\
\text { Accretion Colu } \\
\text { Chromosphere } \\
\text { Gas Fluoresce } \\
\text { Photoevaporat } \\
\text { Jet Bow Shock } \\
\text { HII Region dus }\end{array}$ & $\begin{array}{l}\text { ns } \\
\text { ce/Absorption } \\
\text { distribution }\end{array}$ & $\begin{array}{l}\text { Optic } \\
\text { Accretion Columns } \\
\text { Inner Disk Wall } \\
\text { Jet Acceleration } \\
\text { Jet Collimation } \\
\text { Resolved Disk Imaging } \\
\text { Binary Disk Interactions }\end{array}$ & $\begin{array}{l}\text { cal } \\
\text { Photoevaporation } \\
\text { Jet Rotation } \\
\text { Cooling Distances in Jets } \\
\text { Jet Bow Shocks } \\
\text { Disk Imaging/Detection } \\
\text { HII region cavities }\end{array}$ \\
\hline
\end{tabular}


Figure 5.5.2 - Spatial scales associated with star formation regions at different distances. Each example is the closest for its category. For example, the closest region with accretion disks is Taurus/Auriga. Regions to the right contain objects like those on the left. That is, Carina has several clusters of O-stars each of which resembles Orion, and Orion has many accretion disks like those in Taurus, and Taurus has gas disks like TW Hya. The colors refer to the depictions in Figure 5.5.1.

\section{Spectral Resolution}

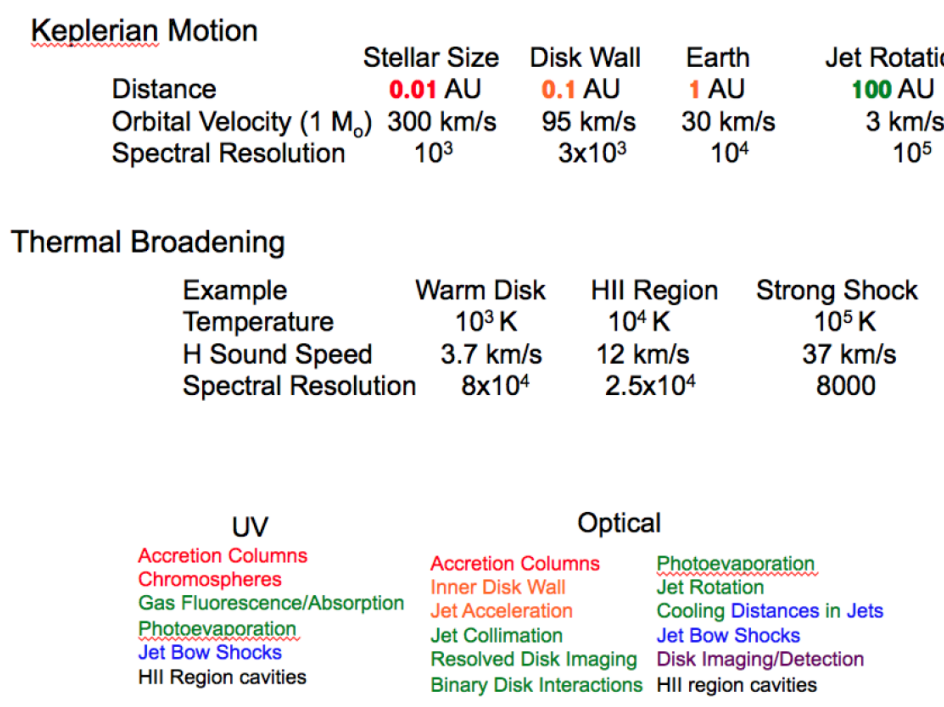

Figure 5.5.3 - Similar to Figure 5.5.2, but for spectral resolution. The colors refer to size scales in Figure 5.5.1.

\subsection{Solar System Astronomy with a Large Aperture Space Telescope - Walt Harris}

Our solar system is the best-studied example for planet system evolution, starting from a protoplanetary disk to the end state planetesimal reservoirs, planetary types and location, and the evolution of the system via migration, tidal forces and solar forcing. Remote sensing has been enormously useful both for improved understanding of the individual objects at the system-level and for probing their origins and subsequent evolution. Study at the level of systems, including atmospheres, satellites, magnetospheres, and their mutual interactions with each other and the Sun, is key to narrowing the parameter space of planetary formation and how their modern states respond to their external environment. This can be summed up with a series of grand questions.

1) How does the interaction with the Sun and Solar Wind affect the movement of mass, momentum, and energy across boundaries in a planetary system?

2) How do solar driven energetic processes affect atmospheric structure, composition, dynamics, and evolution?

3) How does the presence and/or release of internal energy affect surface process, atmospheric dynamics, and magnetospheric environment? 
4) How does the solar driven Earth system compare with the other planets?

5) What was the initial volatile composition of planets, their satellites, and other small bodies and how have they changed with time?

Space based remote sensing of the solar system is an important complement to robotic visitation by virtue of the scale of study it provides. The number and diversity of potential targets in the solar system means that there are far more objects of compelling interest than can be visited in situ. Thrust capacity restrictions limit the number of objects that can be visited quickly and/or co-orbitally, which means that most encounters must be either fly-by events (e.g. New Horizons) or require a long cruise phase (e.g. MESSENGER, Rosetta). Even for cases where spacecraft spend an extended period near an object, they are typically limited in the type and capabilities of the instruments provided, and often by their limited perspective relative to the entire system under study. Global monitoring of multiple bodies is enabled from remote sensing, with relative quality of the observations dependent on the aperture size of telescope used.

The ultraviolet to near-infrared spectral range is particularly valuable for remote sensing of solar system objects because it encompasses the spectral ranges dominated by the solar continuum (300-3000 $\mathrm{nm}$ ) and emission line dominated energetic emissions from the Solar and planetary environments. Reflected visible continuum provides high signal to noise measurement of atmospheric dynamics, surface characteristics and composition, and near space volatiles in magnetospheres and comets. The continuum-free UV extends access to fainter emissions from atmospheric airglow, space weather, auroral processes, exospheric and cometary atomic species, magnetospheric plasmas, and upper atmospheric structure.

A diffraction limited $10+$ meter class observatory would have 20x or greater sensitivity than the Hubble Telescope and would be capable of resolving features as small as 8 to $230 \mathrm{~km}$ over distances from 1 AU out to the orbit of Pluto. With this combination of features, such a telescope would be able to deliver image quality comparable to or better than that achieved with the Voyager spacecraft throughout the region of the solar system occupied by the major planets. The different regions of its spectral range would enable a variety of detailed studies that would exceed combination of temporal and spatial coverage that has been possible to date.

\subsubsection{Plasma Processes:}

Ultraviolet emissions from planetary aurorae, airglow, and magnetospheric plasmas has been a staple method for monitoring energetic processes in planetary atmospheres and their response to solar activity. The Space Telescope Imaging Spectrograph (STIS) is currently the workhorse of these studies, and will continue to provide supporting remote sensing data after existing missions (e.g. Cassini) are complete and as new missions (e.g. JUNO) begin. STIS has proven effective at monitoring emissions from Mars, Jupiter (Figure 1), and Saturn, but is insufficiently sensitive to emissions at Uranus, Neptune, and diffuse magnetospheric sources. Combined with improvements in detector efficiency and dynamic

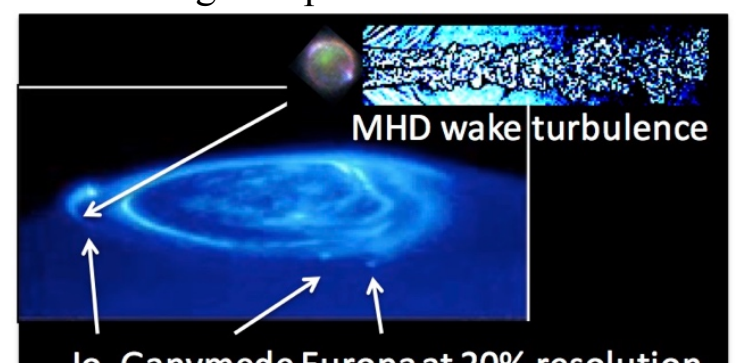
range, a large space telescope will increase sensitivity by 100 fold to enable rapid acquisition of auroral, airglow, and plasmasphere measurements throughout the outer solar system.

Figure 5.6.1 - STIS observations of the Jovian auroral at $20 \%$ of the predicted next generation resolution show dawn-dusk signatures of interaction with the solar wind. 
In addition, field aligned current interactions with Io, Europa, and Ganymede are revealed from 'footprints' South of the main oval. Inset shows the auroral signature of Io's interaction with Jupiter. Observable wake turbulence in the Io torus is simulated at right.

\subsubsection{Surfaces and Activity:}

Signatures of ices, minerals, and activity on planet, satellite, and small bodies can be observed spectroscopically across the full spectral range of a large aperture telescope. Current detection limits are limited to small bodies in the inner solar system and unresolved structures on larger satellites of the giant planets. The volatile surface composition of small bodies has been only sparsely examined with current technology.
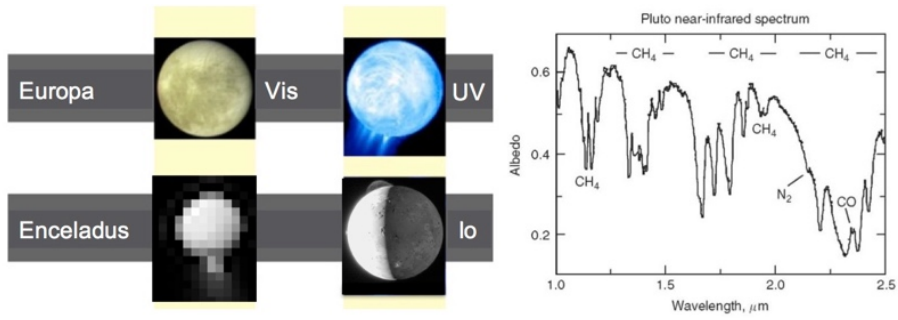

Figure 5.6.2 - Examples are shown of the surface detail detectable on several planet satellites, along with geyser/volcanic activity. NIR observations can obtain surface ices on objects as small as $10 \mathrm{~km}$ in radius (Grundy et al. 2013).

\subsubsection{The Outer Solar System Inventory:}

The Hubble Telescope is currently the most sensitive instrument for small body detection in and beyond the Kuiper Belt. A future $10 \mathrm{~m}$ class observatory should retain that advantage, even over $30 \mathrm{~m}$ ground based facilities because of the reduced background. Such an observatory would enable detection of $\sim 1 \mathrm{~km}$ sized objects throughout the Classical Kuiper Belt and spectroscopic characterization of objects up to $10 \mathrm{~km}$. The range over which Dwarf planets $(\mathrm{R} \sim 500 \mathrm{~km})$ would be detectable would extend to the perihelion of the Sedna-class objects. Possible larger planets ranging in size from Super-Earths to Neptune-sized could be detected out to distances extending into the inner regions of the Oort Cloud (5000-15000 AU).

\subsection{The Galactic Baryon Cycle: CGM Drivers for a Large UV-Optical-IR Space Telescope - Chris Howk}

The flows of matter between galaxies and their surroundings are thought to be critical to the overall evolution of a galaxy. A galaxy's star formation and even AGN activity is coupled to the rates and manners in which gas flows away from and into the system. This includes exchanges of matter within the disk-halo interface of a galaxy, between the central regions of a galaxy and its extended gaseous corona, between the galaxy's halo and its satellites or group, and between a halo and the filamentary IGM beyond.

Understanding gas flows through the circumgalactic medium (CGM) is thus a crucial piece of the whole picture of galaxy evolution. We have seen over the last few years the huge impact new capabilities for studying the CGM can have, as the unprecedented UV sensitivity and good resolution of the Cosmic Origins Spectrograph (COS) have led to some extraordinary new insights into (and unanswered questions about) the CGM about $z \lesssim 0.5$ galaxies and its role in their evolution: 
1. The CGM about $\mathrm{z} \lesssim 0.2$ hosts a large share of galactic baryons, with $M_{C G M} \sim M_{\star}$ for $\sim \mathrm{L}^{*}$ galaxies (Stocke et al. 2013; Werk et al. 2014). When were those baryons first put in place?

2. The CGM is a massive reservoir of galactic metals, with galaxies having ejected at least as much metal mass as they have retained (Tumlinson et al. 2011; Peeples et al. 2014; Bordoloi et al. 2014). What mechanisms drive these metals outward? are they destined to be recycled back to the ISM (Keeney et al. 2013; Ford et al. 2014)?

3. Dense, ionized streams of gas in the low-redshift CGM of galaxies have a bimodal metallicity distribution, with roughly half having metallicity $\sim 20-100 \%$ solar and half exhibiting extremely low metallicities of $<5 \%$ solar (Lehner et al. 2013, Wotta et al. 2016). The low-metallicity gas resembles the long-sought "cold accretion" entering galaxies from the IGM (e.g., Kereš et al. 2005, Nelson et al. 2015). However, does the presence of metal-poor streams affect the starforming properties of galaxies?

4. Galaxies can drive complex multi-phase superwinds containing as much mass as the cold ISM with $\mathrm{T} \sim 10^{4}-10^{6} \mathrm{~K}$ that flow deep into their halos (Tripp et al. 2011). How common are such superwinds? do they require recent AGN activity? How are these related to the more quiescent winds seen in $\mathrm{z} \sim 0.5-1$ and higher galaxies (e.g., Du et al. 2016, Rubin et al. 2014).

5. There is significant cold, bound gas surrounding passive galaxies at $\mathrm{z} \sim 0.2$, perhaps as much as seen in their star-forming counterparts (Thom et al. 2012). What keeps this gas from forming stars? how does the CGM affect (or is affected by) the quenching of star formation? Why are the covering factors of metal lines so much lower (Tumlinson et al. 2011, Huang et al. 2016)?

A future large telescope with FUV capabilities provides a path to answering many of these questions. With such a facility, we would:

Map the ionization state and metallicity of the CGM for various types of galaxies: Our current "maps" of the CGM are largely statistical (e.g., Tumlinson et al. 2011, Borthakur et al. 2016); very few individual galaxies having multiple probes of individual galaxies (Keeney et al. 2013, Chen et al. 2014, Lehner et al. 2015). At the same time, our ability to study even statistically the CGM of rare, but critical, types of galaxies such as extremely massive systems, post-starburst galaxies, or AGN host galaxies is limited by the small surface densities of QSOs on the sky that are bright enough for UV absorption line spectroscopy with COS. Figure 6.1 demonstrates that non-linear gains can be made in the number of QSO-galaxy pairs able to be targeted just by moving to larger apertures and/or enhanced system throughput. A future LUVOIR would facilitate "mapping" the CGM of individual galaxies by using absorption line spectroscopy toward multiple QSO or even background galaxy sight lines; it would push our ability to study the CGM into the epoch of galaxy transformation, testing the putative connection between the CGM and galaxy quenching; it would hopefully allow emission line imaging or multi-object spectroscopy to elucidate the morphology of the flows of energy and matter through galaxies’ CGM (see §5.10). As shown in Figure 6.2, the majority of ionization, metallicity, and mass diagnostics are found in the FUV or EUV. Capturing the CGM diagnostics over a full range of temperatures/ionization states for a broad redshift range requires good UV sensitivity over the range $1000 \AA \leq \lambda \leq 3000 \AA$, if not to the Lyman limit. Point source spectroscopy should reach $R \geq 20,000$; a capability for $\mathrm{R} \geq 75,000-100,000$ for local sources (Local Group) is preferred.

Connect the CGM to IGM filaments and the general environment of galaxies: What is sometimes left unsaid is the assumption that flows in and out of galaxy halos imply the transport of matter across 
the virial radius. A future LUVOIR facility with a moderate resolution $(R \sim 5,000$ to 10,000$)$ survey mode, preferably coupled to a multi-object capability, could provide the path to connecting the CGM properties of galaxies directly to the cosmic web through absorption line spectroscopy, as well as crudely map the CGM itself. One goal of such work would be to study the interaction at the CGM/IGM interface, which may reside near the virial radius, depending on galaxy mass and coronal structure (Nelson et al. 2015).

Probe the structure, metal content, and energetics of galactic winds from their origins in the diskhalo interface of galaxies to the radius at which they stall (if they do): Understanding the driving of flows through the disk-halo interface (the interstellar thick disk), to the galactic corona/CGM, and potentially further will take a combination of multi-object absorption line spectroscopy of the potential launch points of disk-halo flows in nearby galaxies (OB stars/associations) and absorption+emission spectroscopy of wind diagnostics in cosmologically-distant galaxies (both "down-the-barrel" and toward background sources). Understanding the dynamics of such outflows, especially through the CGM, likely requires that we both probe the driving fluids (seen in the very high ions - e.g., Ne VIII and $\mathrm{Mg} \mathrm{X}$ - and through X-rays with Athena) and trace the energetics (both the thermal balance through cooling as well as the kinematics).

Ultimately the groundbreaking observations required to make better sense of the role of the CGM in galaxy evolution involve studying specific stages of galaxy evolution with a full range of ions, which drives the need to do absorption line spectroscopy in the FUV, down to at least $1000 \AA$, and perhaps to the Lyman limit. Falling short of this would make it impossible to study the highest-ionization (in some cases hottest) CGM gas and the probes of energy balance in the CGM. Perhaps the most impressive leaps, however, will come from an imaging or spectral slicing capability that allows us to understand the morphologies of streams about galaxies, as discussed in Section 5.10.

\subsection{Motivations for a future UV-visible telescope: High-redshift Galaxy and Deep-field Studies - Marc Rafelski}

When considering high redshift galaxy surveys in the context of a future UV-optical space telescope, it is important to consider what other dedicated missions will already have accomplished. The two largest such missions include the James Webb Space Telescope (JWST) and the Wide-Field Infrared Survey Telescope (WFIRST). The JWST will survey small areas of the sky with a large collecting area at high resolution in the near-infrared through mid-infrared, while the WFIRST will likely survey a large area of the sky at low resolution in the near infrared. Therefore, future high redshift galaxy survey missions must consider this area of parameter space as already addressed, and consider questions that cannot be accomplished in the infrared or at the resolution of these telescopes.

A large optical-ultraviolet (UV) space telescope will have 5 times the field of view (FOV), 5 times the resolution, 25 times the sensitivity of the Hubble Space Telescope (HST), and cover the UV through optical wavelengths not accessible with other telescopes. These properties will enable such a telescope to answer questions not possible with JWST or WFIRST, as well as new questions that will be asked with their future scientific results. JWST and WFIRST will excel in studying galaxies at the earliest times approaching reionization, and the older stellar populations during the peak epoch of star formation by measuring the rest-frame optical light of galaxies. However, these telescopes are unable to measure the rest-frame UV light from galaxies in the last 10 billion years of the universe. 
The rest-frame UV light measures a different stellar population - the young massive stars from the starforming regions of galaxies at the peak epoch of star formation and thereafter. Measurements of this light would enable us to address many important questions, including four addressed here: 1) the role of sub-galactic clumps in the build-up of galaxies 2) the properties of low-mass galaxies as a function of environment 3) the Lyman continuum escape fraction and 4) the role of neutral HI gas via Damped Lyman-alpha systems (DLAs) in the formation and evolution of galaxies.

The resolution of the proposed telescope enables the measurement of sub-galactic clumps at scales of $\sim 100 \mathrm{pc}$ to learn about their role in the assembly of galaxies. We wish to measure the lifetime of these clumps, whether they form in the centers or edges of galaxies, if they migrate over time, and if they are connected to the accretion from the cosmic web. These clumps can only be studied in the rest-frame $\mathrm{UV}$, as they are young star forming regions. In addition, the rest-frame UV light is necessary to break degeneracies in physical parameters obtained from stellar population modeling, such as mass, age, dust extinction, and star formation rates (SFRs). While JWST will be able to measure them at the earliest times in the universe, those galaxies are not yet sufficiently developed, and due to the cosmological scale factor and a smaller mirror, their effective resolution is significantly worse. Moreover, studying the galaxies at lower redshifts increases the sample time frame for measuring the evolution of the clumps over time. Measurements of the clumps later in time will in turn enable interpretations of galaxies measured by JWST earlier in time.

The large FOV and large collecting area enables the proposed telescope to survey a large area of the sky down to exquisite depths, which will for the first time allow studies of lower mass galaxies at the peak epoch of star formation. The diverse star formation histories (SFHs) of dwarf galaxies suggest that environment plays a key role in the development of low mass dwarfs, and therefore we need large samples of these very faint galaxies in different areas on the sky. While we will obtain redshifts for massive dwarf galaxies with JWST and WFIRST, large samples of low-mass dwarf galaxies will still rely on photometric redshifts, for which rest-frame UV light is essential to remove redshift degeneracies. In addition, simulations predict that low-mass dwarf galaxies have very bursty star formation, which would result in a large scatter in the rest-frame UV to H- $\alpha$ ratio. Therefore the SFRs of such galaxies measured purely in H- $\alpha$ with JWST and WFIRST will not reveal the SFHs of these galaxies, and the physical parameters from stellar population modeling that depend on the SFHs. On the other hand, measurements of the rest-frame UV to $\mathrm{H}-\alpha$ ratio and the dispersion in the rest-frame UV will inform us of the SFH of the galaxies, and enable the measurement of individual star-forming regions within the dwarf galaxies.

JWST will do an exquisite job of studying galaxies just after reionization, yet it will likely not be able to determine what actually reionized the universe. The light that reionized the universe is at wavelengths bluewards of the Lyman-limit, and therefore in the rest-frame UV. While JWST measures this light from galaxies right after reionization, the opacity of the intergalactic median (IGM) absorbs the light before it reaches us. Therefore, the Lyman continuum escape fraction necessary to understand reionization is best measured at later times, which requires an UV to optical telescope. Currently, HST has measured only limits on the escape fraction, suggesting that it is small, and therefore requires deeper imaging. In addition to a detection of the escape fraction, we wish to characterize the physical parameters of galaxies as a function of their escape fraction. For instance, low-mass dwarfs may have a much higher escape fraction than their more massive cousins. This therefore requires very deep imaging over a large FOV. 
The role of neutral atomic hydrogen gas in the formation and evolution of galaxies is currently poorly constrained, yet at early times in the universe it provides most of the fuel for star formation. At this time, most of this gas resides in DLAs, yet we know relatively little about their sizes, morphology, and SFRs since they are only observed in absorption to background quasars rather than in emission. These quasars then prevent direct imaging of DLAs by providing a very bright object right at the location one would wish to observe the faint DLAs. One method to overcome this challenge is to select systems with two DLAs, where the first one blocks all light from the quasar blueward of its Lyman limit, enabling direct measurements of the second DLA before its Lyman limit. The emission from these systems is extremely faint and has not been detected with HST, and the wavelength window enabled by this physical setup shrinks earlier in time. Therefore, a UV-optical telescope capable of measuring very low levels of emission is needed. Such measurements would for the first time inform us of the sizes, morphology, and SFRs of the DLA host galaxies, which would tell us about the cold gas reservoirs fueling star formation.

These four scientific questions require a telescope sampling UV through optical light at high resolution with a large FOV. These requirements are met with a large $(\sim 10 \mathrm{~m})$ UV-optical space telescope, which would enable large imaging surveys providing new insights into the formation and evolution of galaxies at the peak epoch of star formation.

\subsection{The First Stars and Heavy Metal Production - Ian U. Roederer}

Understanding the origin of the elements is one of the major challenges of modern astrophysics. This is expressed in several of the Cosmic Origins science goals. The approach advocated here is based on obtaining high $\mathrm{S} / \mathrm{N}$ and high spectral resolution data of late-type (F-G-K) stars to infer their detailed abundance patterns. This chemical information can then be related back to the physics of element production in early supernovae or other stellar sites. This approach was not really

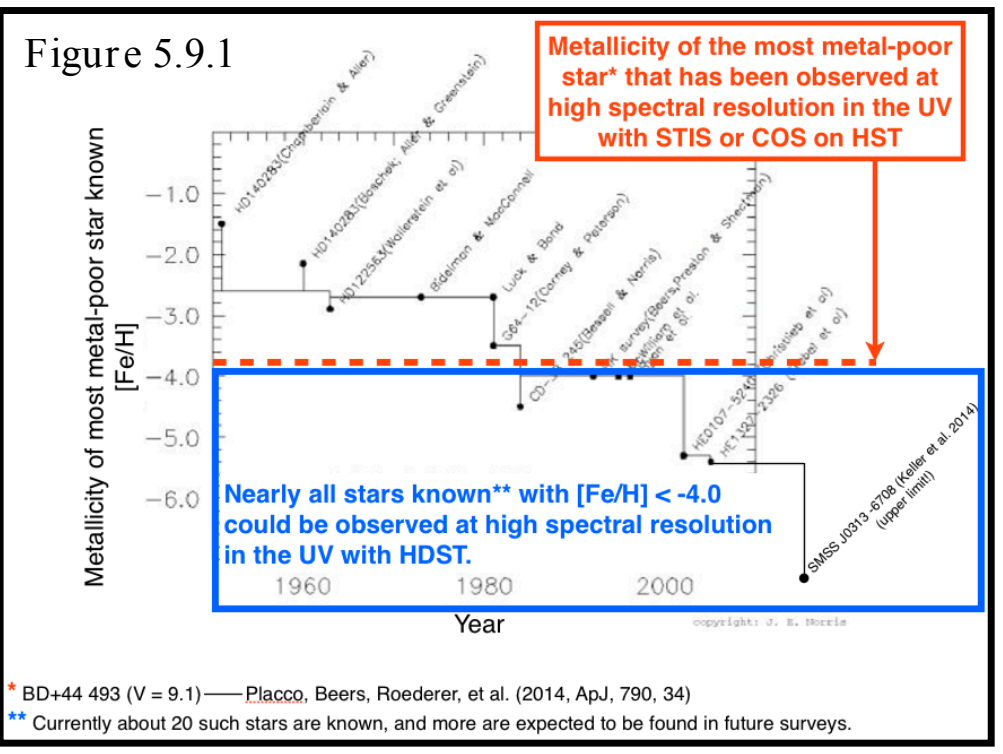
covered by the AURA report.

Our group and others have used HST to demonstrate that UV data can be collected and that they are useful for understanding stellar nucleosynthesis and Galactic chemical evolution. The challenge, of course, is that with HST we are limited to the very brightest stars. Figure 5.9.1 illustrates the situation. The lowest-metallicity star that has been observed at high spectral resolution with HST in the UV sits at the red dashed line. All of the metallicity regime below that will likely remain completely unexplored during the era of HST. But, with something like the High Definition Space Telescope (HDST), you could observe nearly all of the lowest-metallicity stars known. There are about 20 such stars known at present, and we expect that more will be found in future surveys. 
Why is the UV important? The physics of stellar atmospheres dictates that most of the absorption lines are found in the UV, short of the atmospheric cutoff. Figure 5.9.2 illustrates this point for just two narrow spectral ranges, one in the optical on the left, and one in the UV on the right. The top panels show the spectrum of a solar-metallicity G-dwarf; the middle panels show the spectrum of a normal low-metallicity subgiant, and the bottom panels show the spectrum of one of the secondgeneration stars. The bottom right panel is a simulation. Notice how rich the UV spectrum is. In the star shown at the bottom here, a total of 18 metal lines can be detected in the optical; our estimates indicate that approximately 300 metal lines could be detected in the near-UV spectral range. This order-ofmagnitude increase more than doubles the number of elements that can be detected. This is how you make serious

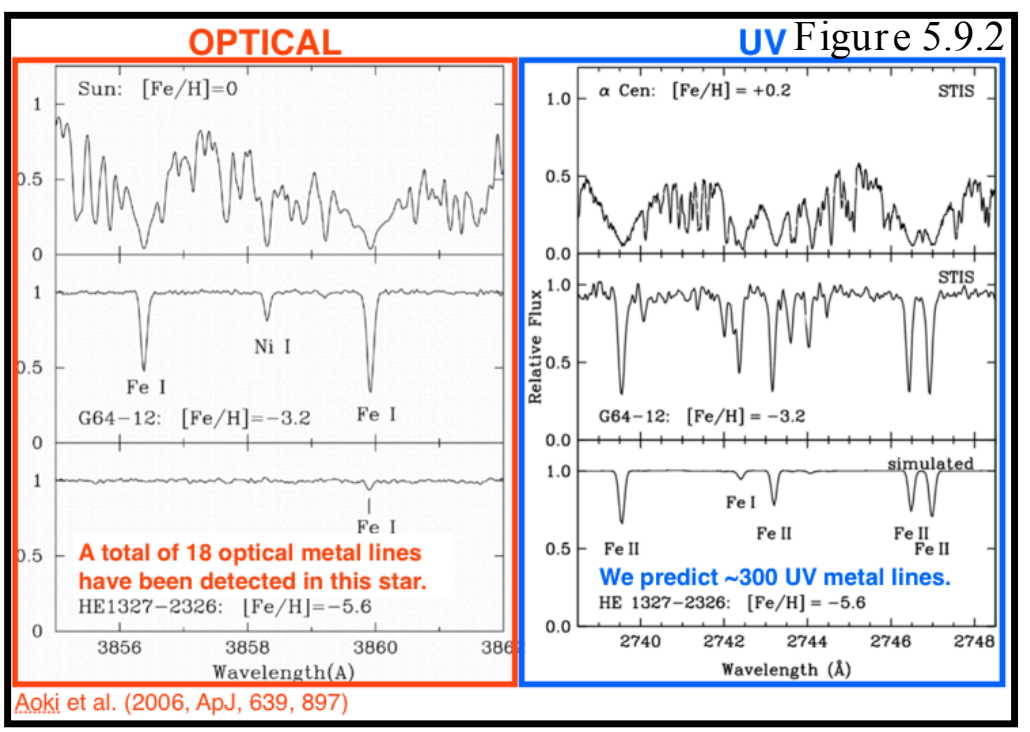
progress in understanding the end states of Pop III stars and the distribution of the first metals in the proto-Milky Way.

The second science case is more focused on understanding the origins of the elements heavier than iron, those formed by neutron-capture reactions like the r-process and s-process. One way to characterize the physical conditions at the nucleosynthesis sites is to study the chemical products. Access to the UV spectrum enables a $40 \%$ increase in the number of heavy elements that can be detected (from $\sim 25$ to $\sim 40$ elements). This includes some of the elements that provide the most sensitive constraints on the nucleosynthesis models. Examples may be found in Roederer \& Lawler 2012.

Currently, this science is limited to stars with $\mathrm{V}<10$ or so, and most viable candidates have been exhausted. In an ideal world, we could observe the "gold standard" stars, the ones that have been extensively studied from the ground and have the most potential to expand our understanding of the neutron-capture physics. Figure 5.9.3 illustrates the situation. With HST, we are limited to observing solar-type dwarfs within about 100 pc and giants to a little beyond 1 kpc. With HDST, you can observe

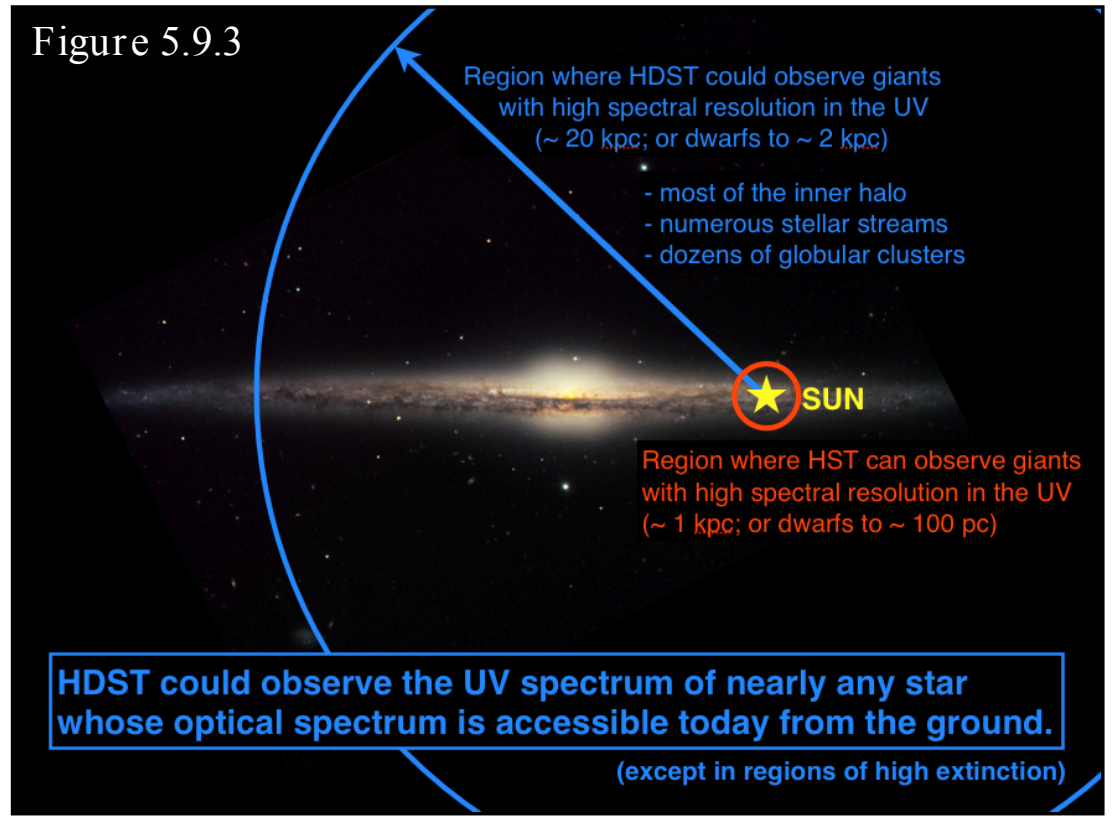


dwarfs to $2 \mathrm{kpc}$ and giants out to nearly $20 \mathrm{kpc}$ in areas of low extinction. That allows you to observe most of the stellar halo, numerous stellar streams including parts of Sagittarius, and dozens of globular clusters. In other words, HDST would give you access to the UV spectrum for just about any star you could observe in the optical today from the ground. That finally puts us in a regime where we get to choose the targets based on astrophysical significance, as opposed to observing whatever happens to be available in the solar neighborhood. With HDST, for the first time we would allow nature to dictate the targets, rather than the limits of our technology.

\subsection{Galaxy Fueling and Quenching: A Science Case for Future UV MOS Capability - Jason Tumlinson and David Schiminovich}

The gas flows that drive galaxy accretion and feedback are critical, but still poorly understood, processes in their formation and evolution. One of Hubble's successes has been in characterizing the circumgalactic medium (CGM) that spans 30 times the radius and 10000 times the volume of the visible stellar disk. Thanks to Hubble and its ground-based optical collaborators, we know roughly how much matter the CGM contains, but the extremely low densities make it difficult to ascertain its exact role in galaxy evolution. The critical question is how this gas enters and leaves the galaxies: galactic star formation rates are limited by the rate at which they can acquire gas from their surroundings, and the rate at which they accumulate heavy elements is limited by how much they eject in outflows. Much of the still-unknown story of how galaxies formed comes down to how they acquire, process, and recycle their gas.

Hubble can make only crude statistical maps by sampling many halos with one absorption-line path through each. The future challenge is to "take a picture" of the CGM using wide-field UV spectroscopy that is $>50 \mathrm{x}$ more sensitive than Hubble. A moderate resolution (R 5000), widefield (3-5 arcmin) multi-object UV spectrograph that can detect this CGM gas directly would revolutionize this subject. This is intrinsically a UV problem since most of the energy transferred by diffuse gas on its way in or out of galaxies is emitted or absorbed in rest-frame UV lines of $\mathrm{H}, \mathrm{C}$, $\mathrm{O}, \mathrm{Ne}$, and other metals, including rest-frame extreme-UV lines that redshift into the 1000-2000 $\AA$ band at $z>0.5$ (Bertone et al. 2013; Figure 5.10.1).

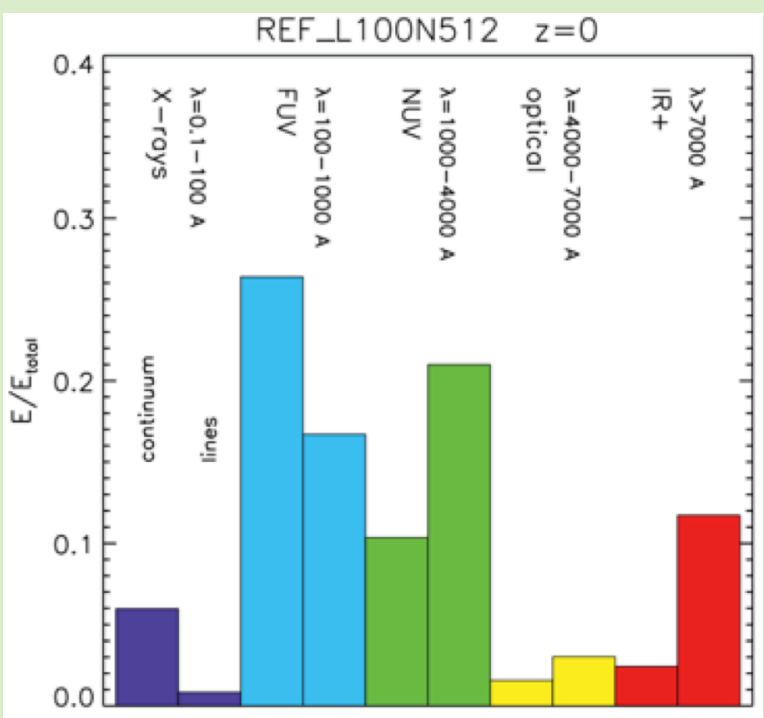

Figure 5.10.1: From "How the Diffuse Universe Cools", by Bertone, Aguirre, \& Schaye (2013). The bars show the fraction of energy emitted in various rest-frame bands by gas cooling radiation in a hydrodynamical galaxy formation simulation. More than half of the radiation is emitted in the rest-frame FUV (100-1000 $\AA$ ) and most of the remainder is in the NUV (1000-4000 ̊).

If sufficiently large (10-12 meters) and properly equipped, NASA's LUVOIR Surveyor will be able to map the density, temperature, and mass flow rates of the CGM, directly, using the UV radiation emitted by CGM gas as it cycles in and out of galaxies. Observing up to 50-100 sources at a time, LUVOIR could map the faint light $\left(\mathrm{S}_{\mathrm{B}} \sim 100\right.$ photons $\left.\mathrm{cm}^{-2} \mathrm{~s}^{-1} \mathrm{sr}^{-1}\right)$ emitted by gas entering and leaving galaxies, to count up the heavy element content of this gas, to watch the flows as they are ejected and recycled, 
and to witness their fate when galaxies quench their star formation, all as a function of galaxy type and evolutionary state. LUVOIR could map hundreds of galaxies in fields where its deep imaging identifies filaments in the large-scale structure, and where ground-based ELTs have made deep redshift surveys to pinpoint the galactic structures and sources of metals to be seen in the CGM.

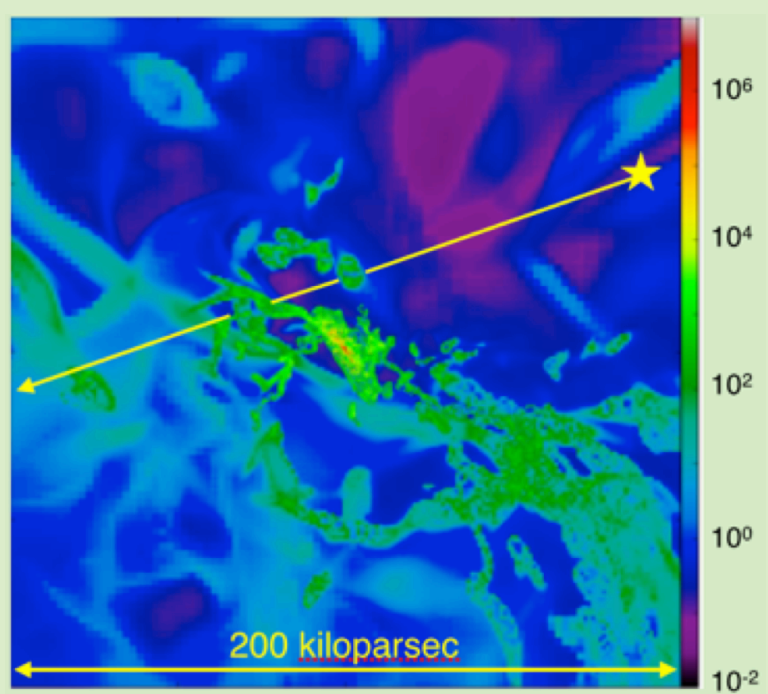

Figure 5.10.2: A visualization of the Circumgalactic Medium (CGM) gas fueling a Milky-Way like galaxy at $\mathrm{z} \sim 0.25$. The color renders the strength of UV emission by $\mathrm{C}$ IV in the CGM gas in terms of photons $\mathrm{cm}^{-2} \mathrm{~s}^{-1} \mathrm{sr}^{-1}$. For real 2D characterization of the CGM, the LUVOIR Surveyor must reach CGM gas with $\mathrm{S}_{\mathrm{B}}$ $\gtrsim 100$ units. The contrast between the $2 \mathrm{D}$ image of the CGM and the single hypothetical QSO sightline demonstrates the power of imaging the CGM over continuing to develop statistical maps from single sightlines. Image from Joung, Fernandez, Bryan, Putman and Corlies (2012, 2015).

Because this radiation is far weaker than local foreground radiation, ground-based telescopes seeking it at redshifts where it appears in the visible $(z>2)$ must perform exquisite sky foreground subtraction to reveal the faint underlying signal. These foregrounds will be considerably lower from space (by 10-100x), shortening required exposure times by an equivalent factor. Even apart from lower sky backgrounds, accessing the UV provides far better access to the relevant line diagnostics over most of cosmic time: the key lines HI Lya, CIV 1550, and O VI 1032 are inaccessible from the ground over the last 10 billion years of cosmic time. This includes all cosmic star formation since its $\mathrm{Z} \sim$ 2 peak, opening for view the complete co-evolution of galaxies and their gas supplies over the period when $\sim 80 \%$ of the cosmic stellar mass density was formed (Madau \& Dickinson 2014).

This unique UV capability will also address the mystery of how galaxies quench and remain so. The number density of passive galaxies has increased 10fold over the 10 Gyr interval since $z \sim 2$ (Brammer et al. 2011). Galaxies undergoing quenching are the ideal laboratories to study the feedback that all galaxies experience: the galactic superwinds driven by supernovae and stellar radiation, the hot plasma ejected by black holes lurking in galactic centers, and the violent mergers that transform galaxy shapes while triggering the consumption or ejection of pre-existing gas. Only a 10-12 meter LUVOIR Surveyor would have the collecting area to support deep, wide-field UV MOS searches for CGM gas at the line emission fluxes that are expected, and the spatial resolution to observe the transformation of star forming disks to passive spheroids at 50-100 pc spatial resolution and closely examine the influence of AGN on this process. For galaxies identified as quenching, emission maps of the surrounding CGM will determine the fate of the gas that galaxies must consume or eject and powerfully elucidate the physical mechanisms that trigger and then maintain quenching. Only a 10-12 meter space telescope can achieve such spatial resolution in the optical and observe the rest-frame UV light necessary to witness the co-evolution of stars and gas in galaxies undergoing this transition. As most of the development of the present-day red sequence occurred since $z \sim 2$, and the key diagnostics are rest-frame UV lines, this critical problem is a unique and compelling driver for a 10-12 meter LUVOIR Surveyor mission in future decades. 


\subsection{Lyman Continuum Luminosity Function Evolution of Star-Forming Galaxies - Stephan McCandliss}

Science Motivation - The timing and duration of the Epoch of Reionization is crucial to the subsequent emergence and evolution of structure in the universe (c.f. Madau et al. 1999, Ricotti et al. 2002, Robertson et al. 2015). The relative role played by star-forming galaxies, active galactic nuclei and quasars in contributing to the Metagalactic Ionizing Background (MIB) across cosmic time remains uncertain. Deep quasar counts provide some certainty to their role, but the potentially crucial contribution from star-formation is highly uncertain due to our poor understanding of the processes that allow ionizing radiation to escape into the intergalactic medium (IGM). Moreover, the fraction of ionizing photons $\left(\mathrm{f}_{\mathrm{LyC}}^{\mathrm{e}}\right)$ that escape from star-forming galaxies is a fundamental free parameter used in models to "fine-tune" the timing and duration of the reionization epoch that occurred somewhere between 13.4 and 12.7 Gyrs ago at redshifts between $12>\mathrm{z}>6$.

Galaxy luminosity functions at high redshift (Bouwens et al. 2006; Labbe et al. 2010; Gonzalez et al. 2010; Finkelstein et al. 2014) along with a host of assumptions for the clumping factor, the ionizing output and the initial mass function of the first stellar assemblages, have been used to constrain $\mathrm{f}_{\text {LyC }}^{\mathrm{e}}$ to 0.2, fulfilling the requirement to power the EoR - provided contributions to the LyC from the unobserved population of galaxies at the faint end of the luminosity function are included. Of all these assumptions, the uncertainty in $\mathrm{f}_{\text {LyC }}^{\mathrm{e}}$ is universally acknowledged as the least understood parameter (Ellis 2014), requiring observation for quantification.

Ionizing radiation escape is a mysterious process. Heckman et al. (2001) have pointed out that mean galactic column densities for H I range from $10^{21} \mathrm{~cm}^{-2}$ for normal galaxies to $10^{24} \mathrm{~cm}^{-2}$ for nuclear starbursts. Yet it only takes a H I column density of $1.6 \times 10^{17} \mathrm{~cm}^{-2}$ to produce an optical depth $\tau=1$ at the Lyman edge. Escape from such large mean optical depths requires that the galaxy interstellar medium (ISM) to be highly inhomogeneous, peppered with low neutral density, high ionization voids and chimneys created by supernovae or the integrated winds from stellar clusters. This implies that escape will be extremely dependent on local geometry, requiring resolutions of star cluster sized structures with typical diameters $\sim 30$ to $100 \mathrm{pc}$. Such objects subtend angles of 0.003 to 0.010 arcseconds at redshifts of 2 or 3.

Direct observation of Lyman continuum (LyC) photons emitted below the rest frame $\mathrm{H}$ I ionization edge at $911.7 \AA$ becomes increasingly improbable at redshifts $\mathrm{z}>3$, due to the steady increase of intervening Lyman limit systems towards high z (Inoue \& Iwata 2008). A key project of the James Webb Space Telescope (JWST) is to search for those sources responsible for reionizing the universe. However, neither JWST nor the Wide-Field InfraRed Space Telescope (WFIRST), can address the key question of, "How Does Ionizing Radiation Escape from Star-Forming Galaxies?"

The far-UV and near-UV bandpasses provide the only hope for direct, up close and in depth, detection and characterization of those environments that favor LyC escape. By quantifying the evolution over the past 11 billion years $(\mathrm{z}<3)$ of the relationships between LyC escape and local and global parameters such as: metallicity, gas fraction, dust content, star formation history, mass, luminosity, redshift, over-density and quasar proximity, we can provide definitive information on the LyC escape fraction that is so crucial to answering our key question. Our goal is a definitive determination of L 900 
galaxy luminosity functions over a redshift range from 0 to 3 and will allow us to test whether the escape fraction of low luminosity galaxies is luminosity dependent.
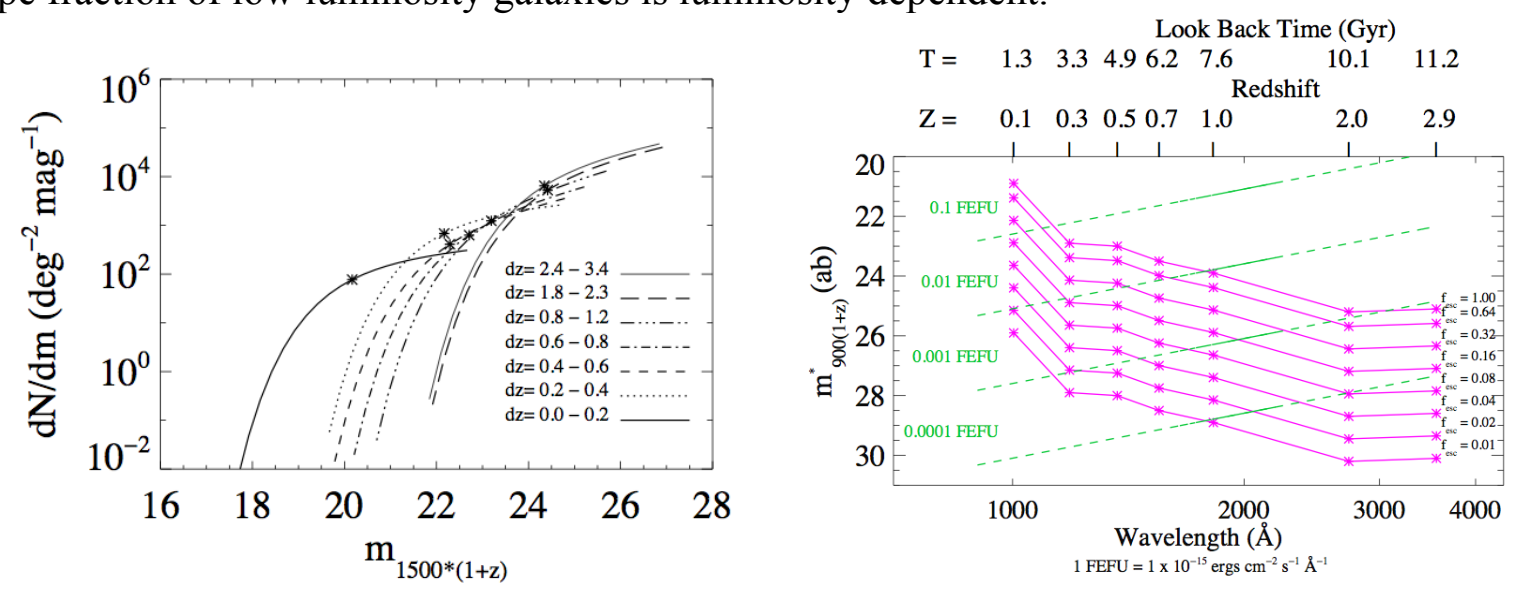

Figure 5.11.1 - Left panel - Surface densities as a function of observer's frame apparent magnitude for galaxy populations with redshifts between $0-0.2,0.2-0.4,0.4-0.6,0.6-0.8,0.8-1.2,1.8-2.3,2.4-3.4$, estimated following Arnouts et al (2005). There are 100s - 10,000's of galaxies per square degree. Right panel- the purple asterisks show the characteristic apparent LyC magnitudes $(\mathrm{ab}) \mathrm{m} * 900(1+\mathrm{z})$ as a function of look back time, and in redshift and wavelength space, for different escape fractions. Contours of constant flux units are overplotted as green dashes marked in FEFU fractions defined in the abscissa subtitle.

Observational Requirements - We have undertaken a study (McCandliss et al. 2008; McCandliss 2012) to estimate the flux detection requirements for escaping Lyman continuum photons from starforming galaxies, as a function of redshift, guided by the galaxy luminosity functions of Arnouts et al. (2005), shown in the left panel of Figure 5.11.1. In the right panel of Figure 5.11.1 we provide ionizing continuum flux estimates for "characteristic" ( $\left.\mathrm{L}^{*} \mathrm{UV}\right)$ star-forming galaxies as a function of look back time and escape fraction. We find ab-magnitudes for $\mathrm{L}^{*}$ uv galaxies of 30 having escape fractions of $1 \%$ between redshift of 2 to 3 . We note that the faint end of the higher redshift luminosity functions are $10 \mathrm{x}$ fainter than $\mathrm{L}^{*} \mathrm{UV}$, so the detection requirements for the faintest galaxies with similar escape fractions will be 10x lower (although some theorist argue that small galaxies should have high escape fractions).

We will take $\mathrm{f}_{900}=10^{-20} \mathrm{erg} \mathrm{cm}^{-2} \mathrm{~s}^{-1} \AA^{-1}$ as a representative flux. This is a challenging flux level to reach, requiring a product of effective area, time and bandpass $\sim 2.5 \times 10^{9} \mathrm{~cm}^{2} \mathrm{~s} \AA$ at $2000 \AA$ to reach a $\mathrm{S} / \mathrm{N}$ of 5 . A telescope with an effective area of $\sim 15,000 \mathrm{~cm}^{2}$, observing for 5 hours with either a filter or spectrograph bandwidth of $10 \AA$ can satisfy this requirement, assuming no significant background. Additional requirements include a sample size exceeding 25 objects per luminosity bin per redshift interval to yield an rms deviation of $<20 \%$ for each point. The total angular area of the sample should exceed $>1$ degree (the characteristic angular scale for BAOs), by a fair margin to beat down cosmic variance. Redshifts are required for each object.

Instrumental Requirements - These observational requirements can be met with a 10 - 12 m class UV telescope with multi-object spectroscopic capability with a spectral resolution of $200-1000$. The diffraction limit for a $12 \mathrm{~m}$ telescope at $2000 \AA$ is 0.003 arcseconds satisfies the spatial sampling requirement. A 2 arcminute wide focal plane at f/24 and $12 \mathrm{~m}$ requires a $170 \mathrm{~mm}$ detector FOV. The TRL for such UV detectors and multi-object spectrographs is TRL 5; for $12 \mathrm{~m}$ space qualified mirrors 
diffraction limited at $2000 \AA$ is likely TRL 1 . Such a telescope could be compatible with a HabitableExoplanet Imaging mission.

\subsection{Characterizing the Habitable Zones of Exoplanetary Systems with a Large Ultraviolet/Visible/Near-IR Space Observatory - Kevin France}

Understanding the surface and atmospheric conditions of Earth-size $\left(\mathrm{R}_{\mathrm{P}} \approx 1-1.5 \mathrm{R}_{\oplus}\right)$, rocky planets in the habitable zones (HZs) of low-mass stars is currently one of the greatest astronomical endeavors. The Astro2010 Decadal Survey ranks the "Identification and characterization of nearby habitable exoplanets" as the premier Discovery Goal for astrophysics at present, with "can we identify the telltale signs of life on an exoplanet?" as a specific focus question. The nearest known Super-Earth mass planets $\left(2-10 \mathrm{M}_{\oplus}\right)$ in the $\mathrm{HZ}$ orbit late-type stars ( $\mathrm{M}$ and $\mathrm{K}$ dwarfs). In addition, all of the $\mathrm{HZ}$ planets found by TESS will be around M dwarfs, making these systems prime targets for spectroscopic biomarker searches with JWST and future direct spectral imaging missions (Deming et al. 2009; Belu et al. 2011).

The planetary effective surface temperature alone is insufficient to accurately interpret biosignature gases when they are observed in the coming decades. The UV stellar spectrum drives and regulates the upper atmospheric heating and chemistry on Earth-like planets, is critical to the definition and interpretation of biosignature gases (e.g., Seager et al. 2013), and may even produce false-positives in our search for biologic activity (Hu et al. 2012; Tian et al. 2014; Domagal-Goldman et al. 2014). Therefore, our quest to observe and characterize biological signatures on rocky planets must consider the starplanet system as a whole, including the interaction between the stellar irradiance and the exoplanetary atmosphere.

Spectral observations of $\mathrm{O}_{2}, \mathrm{O}_{3}, \mathrm{CH}_{4}$, and $\mathrm{CO}_{2}$, are expected to be important signatures of biological activity on planets with Earth-like atmospheres (Des Marais et al. 2002; Kaltenegger et al. 2007; Seager et al. 2009). The chemistry of these molecules in the atmosphere of an Earth-like planet depends sensitively on the strength and shape of the host star's UV spectrum. $\mathrm{H}_{2} \mathrm{O}, \mathrm{CH}_{4}$, and $\mathrm{CO}_{2}$ are sensitive to far-UV radiation (FUV; $100-175 \mathrm{~nm}$ ), in particular the

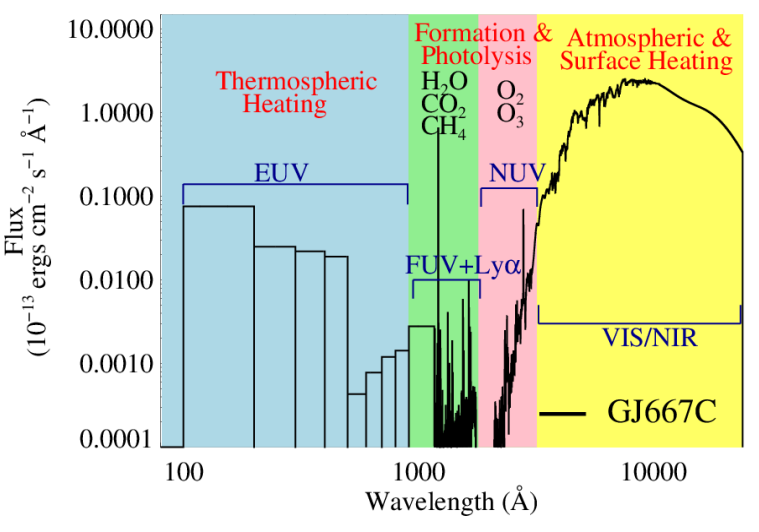

Figure 5.12.1: HST spectrum of GJ 667C, illustrating the influence of each spectral bandpass on the atmosphere of an Earth-like planet orbiting this star (France et al. 2013, Linsky et al. 2014). GJ $667 \mathrm{C}$ hosts a super-Earth mass planet located in the $\mathrm{HZ}$. bright HI Ly $\alpha$ line, while the atmospheric oxygen chemistry is driven by a combination of FUV and near-UV (NUV; $175-320 \mathrm{~nm}$ ) radiation (Figure 5.12.1). Furthermore, the FUV and NUV bandpasses may be promising spectral regimes for biomarker detection (Bétrémieux \& Kaltenegger 2013). High levels of extreme-UV (EUV; $10-91 \mathrm{~nm}$ ) irradiation can drive hydrodynamic mass loss on all types of exoplanets (e.g., Koskinen et al. 2013; Lammer et al. 2014). Ionization by EUV photons and the subsequent loss of atmospheric ions to stellar wind pick-up can also drive wide-scale atmospheric mass-loss on geologic time scales. As the opacity of the ISM precludes direct observations of the EUV irradiance, FUV observations provide the best constraints on the EUV emission from cool stars (Linsky et al., 2014; Shkolnik \& Barman 2014). 
The temporal variability of these sources must also be considered as even weakly-active, "old" (several Gyr) $M$ dwarfs show extreme flare events in their UV light curves (factors of greater than 10 flux increases on time scales of minutes, see Figure 5.12.2; France et al. 2013; Loyd \& France 2014). Impulsive UV events are also signposts for energetic flares, those that are associated with large ejections of charged particles. Energetic particle deposition into the atmosphere of an Earth-like planet during a large $\mathrm{M}$ dwarf flare can lead to significant atmospheric $\mathrm{O}_{3}$ depletions $(>90 \%$ for large flares; Segura et al. 2010). This alters the atmospheric chemistry and increases the penetration depth of UV photons that could potentially sterilize (or catalyze) surface life. Given that particle fluxes cannot typically be directly measured for stars other than the Sun, UV observations offer the best estimates of these important particle environments.

NASA's next large ultraviolet/optical/infrared flagship mission, a LUVOIR Surveyor, could carry out both the direct detection of atmospheric tracers on these worlds and the essential characterization of the star-planet system. The transformative science enabled by a LUVOIR Surveyor to the field of astronomy as a whole, including exoplanet (ExoPAG) and cosmic origins (COPAG) themes, is made clear in NASA's 2013 Astrophysics Roadmap (Enduring Quests, Daring Visions: NASA Astrophysics in the Next Three Decades): a LUVOIR Surveyor (or ATLAST/HDST-like mission; Tumlinson et al. 2015) directly addresses the largest number of core NASA science priorities in a single mission in the next 30 years, working toward six "primary goals", twice as many core science goals as any other intermediate-term mission concept (Section 6.4 in 2013 Roadmap).

There are a set of baseline requirements for the characterization of the energetic radiation environments around low-mass exoplanet host stars. Spectral coverage to wavelengths as short as $102 \mathrm{~nm}$ is crucial to characterizing the star in important activity tracers (e.g., $\mathrm{O}$ VI and Ly $\alpha$ ), as well as peak absorption cross-sections for relevant molecular species in planetary atmospheres (e.g., $\mathrm{O}_{2}$ and $\mathrm{H}_{2}$ ). We would like to characterize typical mid-M dwarf exoplanet host stars out to $30 \mathrm{pc}$ with sufficient signal-tonoise to carry out time-resolved, $R \approx 3000$ spectroscopy of UV emission lines (e.g., N V, Si IV, C IV; $F_{\lambda} \sim 1 \times 10^{-16}$ erg $\left.\mathrm{cm}^{-2} \mathrm{~s}^{-1}\right)$ on the scale of minutes $(\mathrm{S} / \mathrm{N} \sim 10$ per $60 \mathrm{~s})$. These sensitivities can be achieved with a 10 -meter class mirror, optical coatings with broadband UV reflectivity $\geq 90 \%$, and photon-counting detectors with UV DQE $\geq 75 \%$. These technology goals can be achieved with a reasonable investment in laboratory development efforts and hardware demonstration flights (via suborbital and small satellite missions; e.g., France et al. 2012) in the next 5-10 years, enabling the start of a LUVOIR Surveyor mission in the

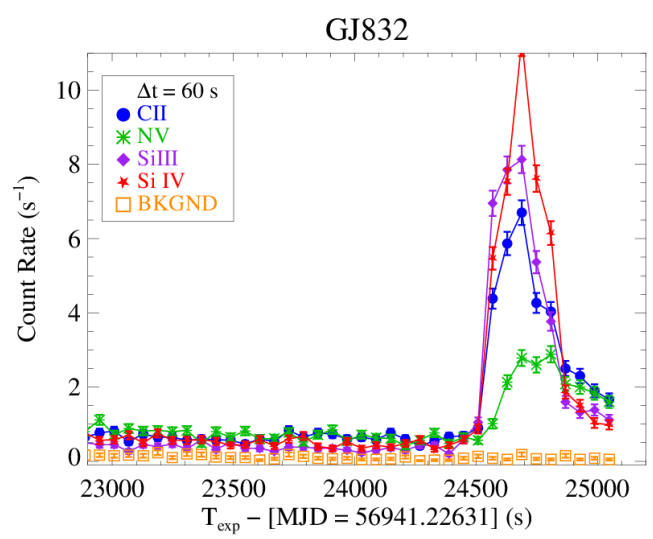

Figure 5.12.2: FUV flare on the "optically inactive" M dwarf GJ 832 (d = 4.9 pc), illustrating a flare event in FUV emission lines on sub-minute timescales (HST-COS data, binned to $60 \mathrm{~s}$ intervals). 2020s. 


\section{Technology}

\subsection{Overview - Matt Beasley}

1. Development of improved coatings for space telescopes allows increased capabilities in wavelength coverage, improved wavefront control, and overall efficiency. New materials with improved control of the deposition process are critical improvements that enable more science by adding targets and creating more capable instruments.

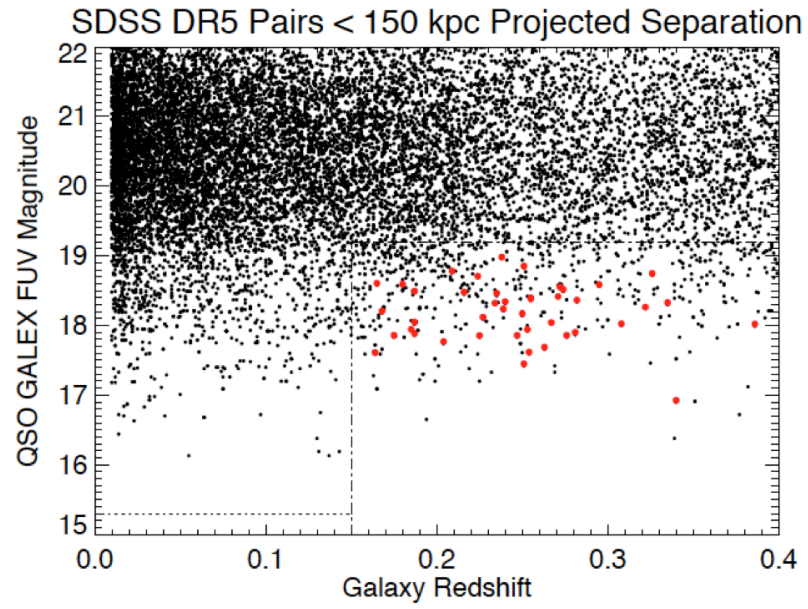

Figure 6.1. From Jason Tumlinson. The dotted line describes the available targets with Hubble-COS and the FUSE spacecraft. The red dots are the targets that have been observed. Clearly, extending Hubble-COS sensitivity toward the blue allows vastly more targets.
Improvements to mirror coatings suitable for large mirrors would contribute to the largest possible gains in scientific return from a Cosmic Origins Mission. First, if ultraviolet reflectivity can be improved compared to existing missions, it will add to the number of scientifically viable targets. In general, the number of targets increases dramatically as a function of minimum detectable flux. (See Figure 6.1) Second, improved coatings improve wavefront error (WFE) budgets. With an optical coating requiring less of the overall WFE, more error can be allocated to fabrication and alignment, which reduces risk and therefore cost of a mission. Third, opening the ultraviolet further toward the blue, changes and improves the fundamental science that can be accomplished. Access to wavelengths currently unavailable (such as 90

- $110 \mathrm{~nm}$ ) provide data that simply cannot be accessed without the increased bandpass. (Figure 6.2) Finally, and it cannot be stated strongly enough, improved reflectivity creates more capable instruments beyond a simple increase in effective area. For example, far-ultraviolet spectrographs can be crossdispersed without a significant loss of effective area, allowing high spectral resolution and broad bandpass simultaneously. That combination, compared to existing systems, reduces the observing time necessary on a target by the multiplex advantage of an echelle - well beyond the simple improved effective area throughput. Moving to multi-object systems could increase the advantage even further.

Beyond improvements to ultraviolet capability, improved coatings would be the path forward to developing systems that would produce acceptable wavefront errors for exoplanet observations. Specifically, dielectric protective coatings may have too much polarization for some current coronagraph designs. Bare metal coatings will have a smaller overall polarization effect, but there are only a few metal coatings suitable for coating a large mirror with associated handling practicalities.

2. Optical / NIR detector development: Optical detectors are nearly perfect for general astrophysical detections. High time resolution is critical for a small number of science cases. Improved detector radiation tolerance will extend the usable lifetime of missions, particularly for missions not in Low Earth Orbit. Leveraging the commercial developments in detectors will add capability to future missions beyond what the limited budget for technology investment can provide. Projects that 
improve the TRL of new detector technology can supply the large focal planes that future origin missions will need to maximize their science return.

\section{Strongest Resonance Transitions Detectable in Interstellar/ Intergalactic Gases (Verner, Tytler \& Barthel 1994)}

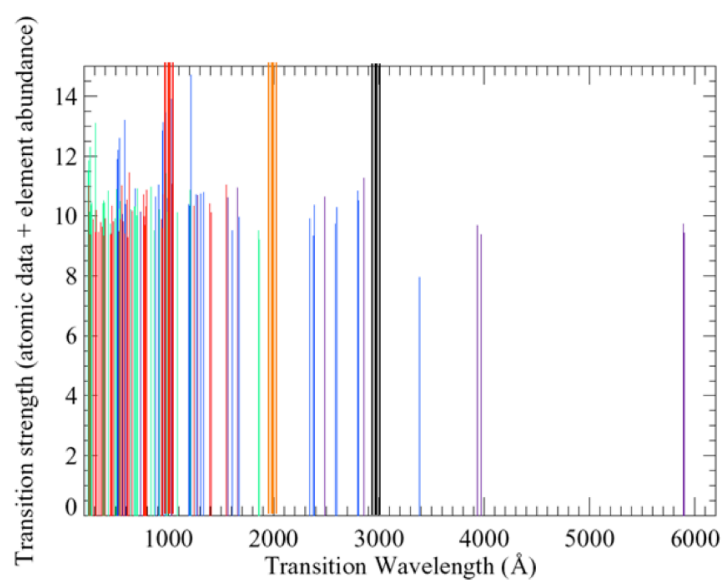

Figure 6.2. Astrophysically relevant lines: there are more below 1000 angstroms than above it (Source: Todd Tripp)

return for any ultraviolet mission.
Making sure NASA leverages commercial detectors, which spends vastly more on R\&D than NASA does, will allow the development of large, high resolution, high dynamic range detector systems. Alternate read schemes combined with the low noise of available sCMOS (scientific CMOS) detectors could allow cosmic ray tolerant imaging, improving the data yield, particularly for missions beyond Earth's magnetosphere.

3. Ultraviolet detectors can be improved for quantum efficiency, resolution, and dynamic range. Extending these capabilities over large area detectors increases the science

More capable UV detectors will leverage the capability of more sophisticated instrumentation afforded by improved ultraviolet reflectivity. Moreover, even without improved reflectivity, moderate to large gains in detector efficiency are still possible for ultraviolet detectors.

4. Mirror technology improvements decrease the cost of missions and improve system capability. Development work that demonstrates technologies for fabrication and integration of mirrors will reduce cost and risk for future missions.

5. NASA should seek to improve opportunities for instrument builders - Nancy Roman fellowships, other design opportunities. While not a direct technology development, increasing the number of practitioners and more novel designs will enable future missions.

\subsection{Detectors for UV-Visible Flagship Missions - John Vallerga}

Choosing the best detector technology for the next UV-Vis. Flagship mission is not obvious, given the recent advances in their performance and technology readiness. However, I will try to summarize the performance of existing technology and mention candidates that have exciting new capabilities if they can establish the resilience of a flight qualified detector. 
First some criteria that most proposed detectors must meet. Larger telescopes with large fields of view require larger detectors (assuming a fixed f-number). For example, an 8 meter $\mathrm{f} / 3$ telescope with a 30 arc minute field of view requires a $20 \mathrm{~cm}$ detector. High quantum efficiency is always desired as its impact on the science scales directly as the telescope area does. The UV sky is dark, so the signal to noise ratio of many science programs is limited by the detector background and/or readout noise. Imaging detectors that have the ability to "photon count", which means detecting the arrival position of individual photons, effectively eliminate readout noise. A detector with high dynamic range is needed to broaden the choice of science targets and overlap previous mission capabilities.

Of course, there are other desirable attributes not directly related to the science performance. The detector should have a long lifetime with respect to the mission duration. It and its support electronics should be radiation hard and preferably have a low operational complexity (e.g. high voltage or cryogenic requirements, vacuum pumps etc.). Response should be uniform and stable for low signal to noise observations. Also, low mass and low power requirements are always desired.

\subsubsection{The Candidates}

\section{Microchannel Plate (MCP) Detectors}

MCP detectors have been the detector of choice for many recent UV instruments (FUSE, COS and STIS on Hubble). MCP detectors count individual photons by amplifying the initial photoelectron by many orders of magnitude and

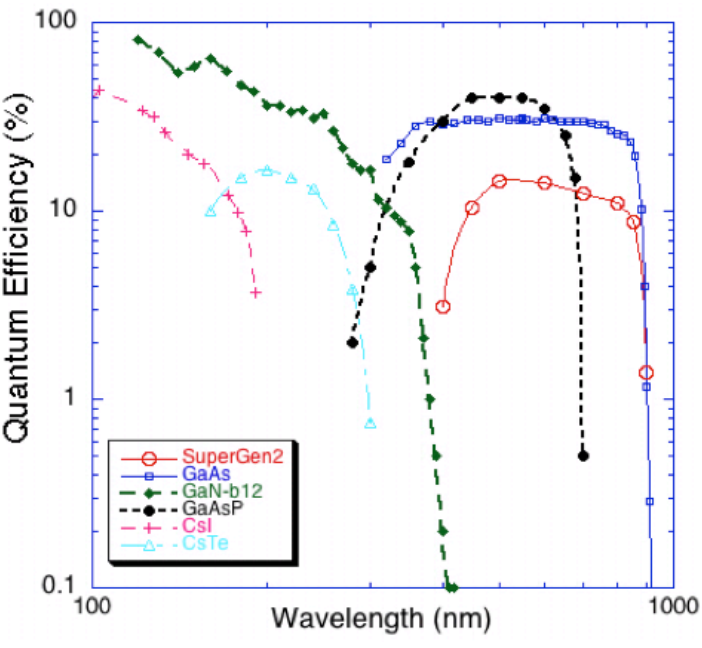

Figure 6.2.1 - Sample of photocathode efficiencies detecting the location of this charge on a patterned anode. The initial photoelectron was released by the incoming photon from a photocathode, either deposited on the MCP itself or on a window directly above the input surface. Hence the QE of such detectors is limited by choice and quality of existing photocathodes (Figure 6.2.1) and tends to be smaller in the optical/IR.

MCP detectors are photon counting, can be made in areas up to $20 \times 20 \mathrm{~cm}$ (Figure 6.2.2), and are inherently rad hard (made of glass). The also can be curved to match curved focal planes, have excellent spatial ( $<20$ micron FWHM) and temporal resolution (sub-nanosecond), low background rates $<0.02 \mathrm{ct} \mathrm{cm}^{-2} \mathrm{~s}^{-1}$. Most UV photocathodes are "solar blind", i.e. have a poor red response.

Some noted weaknesses of this detector technology have been the low NUV/optical QE, limited lifetime (local gain loss) to extracted charge, limited count rate of the electronics, non-uniform response, spatial distortions, and high voltage requirements. Yet recent NASA investments in this technology through the ROSES APRA and SAT programs have achieved (Siegmund et al 2014; Tremsin et al 2013; Vallerga et al 2014) 30\% QE at 200nm, lifetimes of 7 Coulombs $\mathrm{cm}^{-2}$ (order of magnitude higher than predicted mission lifetimes), uniformity better than $3 \%$, count rates $>5 \mathrm{MHz}$ global and $40 \mathrm{kHz}$ local, and spatial

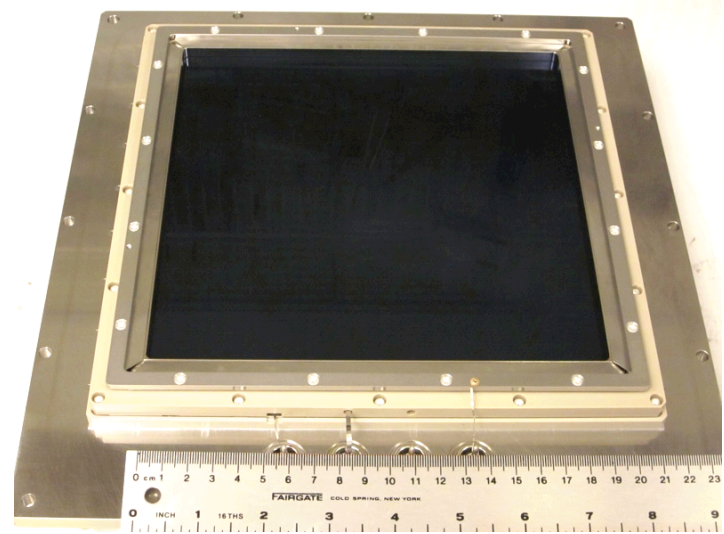

Figure 6.2.2 - A $20 \times 20 \mathrm{~cm}$ photon counting detector using atomic layer deposited MCPs 
distortions $<6$ microns. Given this progress, and its heritage, this is the detector most likely to be qualified for the upcoming UV portion of a UV-Vis flagship mission.

\section{Silicon Detectors}

Detectors based on the absorption of input photons into the silicon bulk where an electron-hole pairs are generated are ideally suited for the optical to near-IR because of their extremely high QE (Figure 6.2.3). Various integrating detectors such as CCDs and CMOS arrays are mature technologies with significant flight heritage (WFC3 on Hubble) and can be tiled into large arrays as well. Until recently, however, they have not been used in the deep UV because of the inability of UV photons to penetrate through the surface layers into the bulk silicon. They must operate at cryogenic temperatures to minimize dark current and must be read out often to minimize cosmic ray hits, thereby taking a readout noise penalty.

Surface treatments, such as delta doping and atomic layer deposition of anti-reflection coatings can overcome the lack of UV sensitivity in specific bandpasses (Hennessey et al 2015; Hamden et al 2014; Hamden et al 2012). Not only will this work on charge integrating CCDs and CMOS devices, but also other silicon technologies that offer potential photoncounting capabilities such as avalanche photodiode (APD) arrays and electron multiplying CCDs (EMCCD). Surface treated EMCCDs will soon be launching on the sub-orbital rocket mission "CHESS" (Hoadley et al 2014) and the FIREBALL-2 balloon payload (Hamden et al 2014).

\section{Microwave kinetic inductance detectors (MKIDs)}

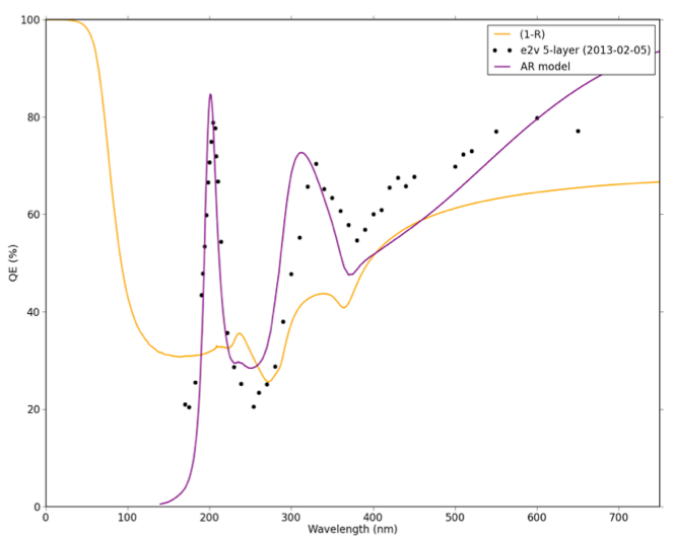

Figure 6.2.3 - QE of photon counting silicon EMCCD optimized for balloon UV window. (Hamden et al 2014)

MKID detectors (Mazin et al 2012) can be thought of as an array of superconducting resonant microwave receivers kept at $100 \mathrm{mK}$ operating temperature. When a photon is absorbed in the pixel, quasi-particles are generated and the inductance change moves the receiver off-resonance. The amplitude and phase of this effect is proportional to the energy absorbed, so the wavelength of the individual photon can be determined to a spectral resolution of $\sim 20$. This brings the advantage of nondispersive spectroscopy to the optical and NIR where 20 colors can be determined in a single pixel with its low background. The MKID detector is photon-counting, has no read noise or dark current and has good QE. Unfortunately, array sizes of 10-20 kpixel are just beginning to arrive at ground based telescopes, though formats of megapixel range are possible. Flight qualified $20 \mathrm{~cm}$ arrays will probably not be ready for the next Flagship mission, in any bandpass.

\subsubsection{Summary}

Quantum efficiency trumps most performance parameters in detector technology. In the far UV $(<$ 150nm), photocathode efficiencies are large enough that MCP detectors with their improved performance in photon counting, large format, resolution, background, lifetime and radiation hardness make them the detector of choice. In the visible/NIR, a silicon CCD array will be chosen, as usual, with its exquisite QE, uniformity and stability. In the near UV, the crossover wavelength from silicon to MCP will depend on the latest QE performance of GaN photocathodes vs. surface treated and coated silicon EMCCDs operated in photon counting mode, assuming both technologies demonstrate their flight worthiness. 


\subsection{Primary Mirror Parameters and Features - Gary Matthews}

The primary mirror for a large UV system such as LUVOIR, ATLAST or HDST will most certainly have to be segmented and one major trade will be the segment size and configuration. For JWST, it turned out that a $1.3 \mathrm{~m}$ flat-flat hexagonal segment was the right answer. Shortly after the award to Northrop Grumman, there were 36 segments of less than $1 \mathrm{~m}$ in size. As the design and analysis process progressed, it was determined that the size was just too small and drove a lot of actuator mass and complexity due to having 7 actuators per segment. By going from 36 segments to 18 segments, the number of actuators was cut in half.

For future UV/Visible systems with a coronagraph in a $12 \mathrm{~m}$ class size, a new trade is required. From a manufacturing perspective, something like the $1.4 \mathrm{~m}$ class segment is advantageous since the segment is easy to handle yet large enough that the edges effects are kept to a minimum.

It is important to first evaluate the science needs with respect to segmentation:

- How do segment edges impact the PSF of the image?

- Does the system need a round outer diameter (Figure 6.3.1) or is a JWST-like (Figure 6.3.2) outside edge acceptable?

- Are there any primary mirror obstructions that are more troublesome than others?

- Does segment size offer significant science return advantages? For example, what if the segments were $5 \mathrm{~m}$ each?

Though there are certainly other factors that need to be considered those listed above should be given initial consideration during the system architecture conceptualization since they may drive the technology development required for the mission. Figures 6.3.1 and 6.3.2 below provide a range of possible solutions.
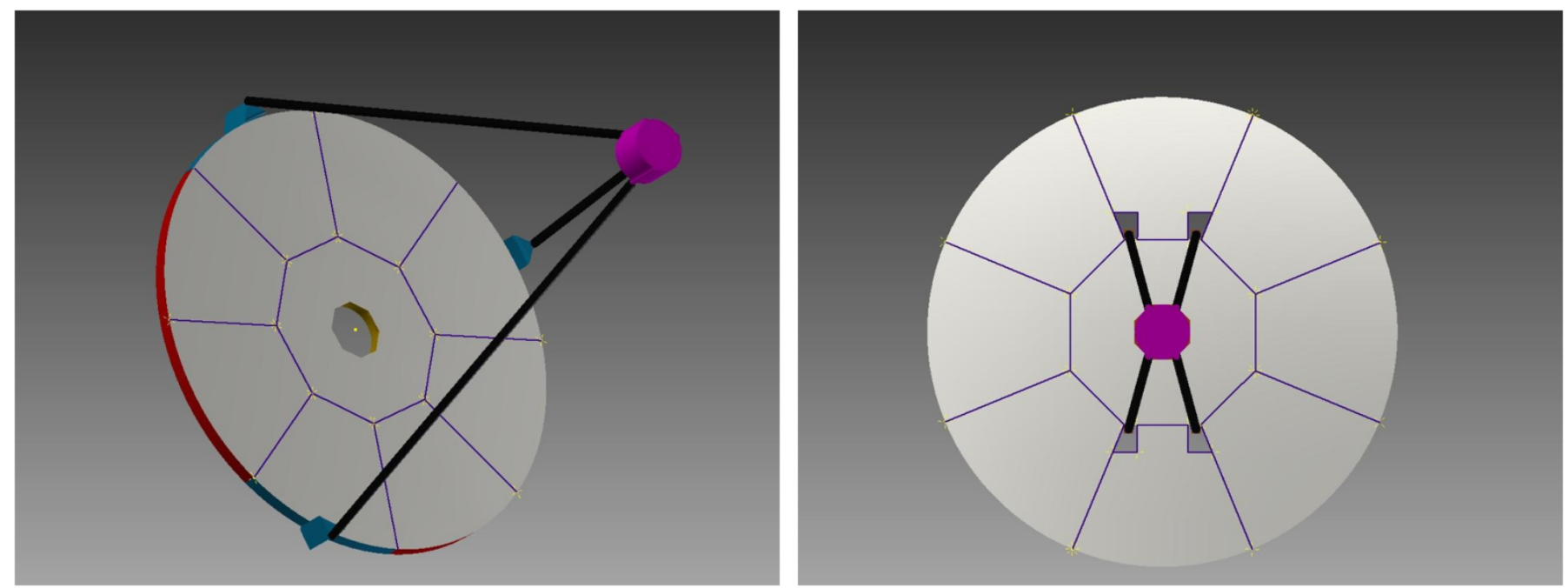

Figure 6.3.1 - These are two examples of $5 \mathrm{~m}$ segments in a $12.7 \mathrm{~m}$ telescope. On configuration has the secondary struts on the outer diameter and the other configuration shows the secondary with in-board struts. There are advantages and disadvantages of each configuration. 


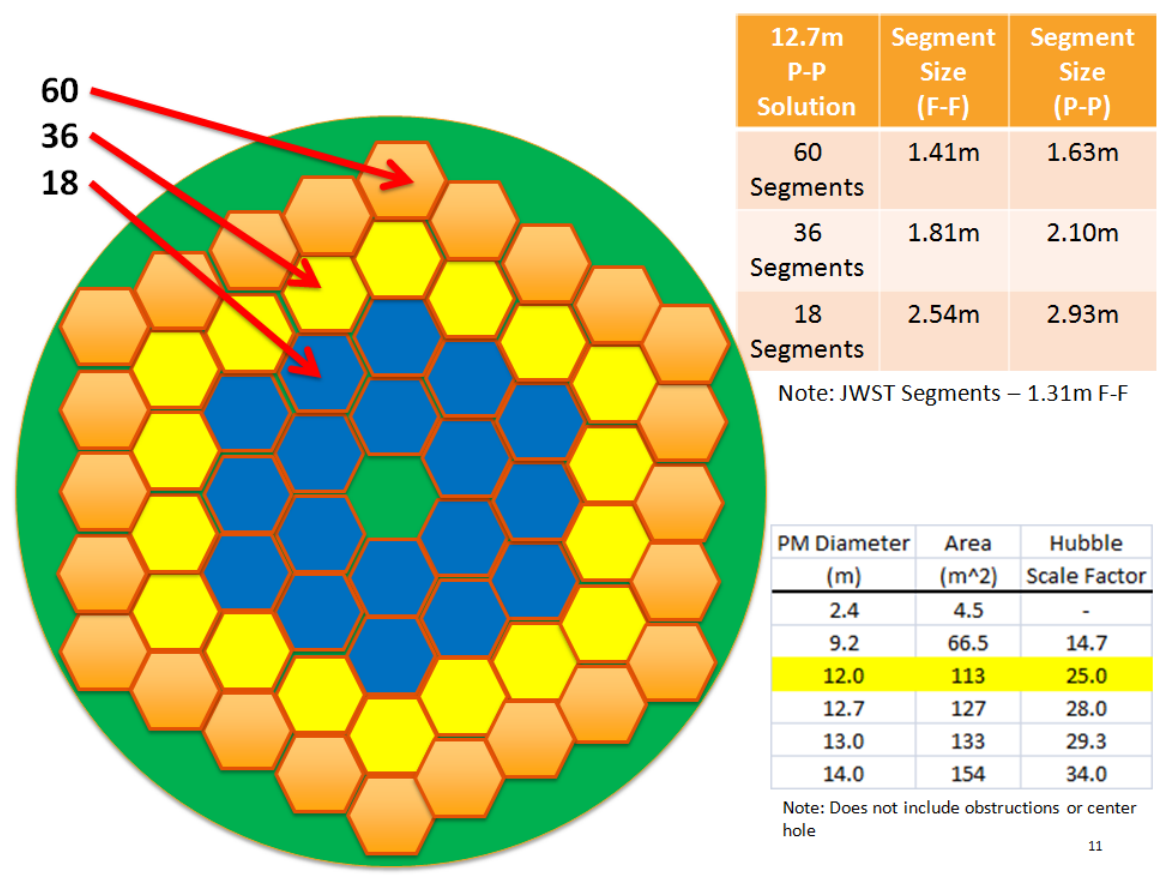

Figure 6.3.2 - This is a JWST-like segmented design. The size of the segments for various segment numbers are shown as compared against JWST. The overall primary mirror collecting area is also shown against HST. As can be seen, a $12 \mathrm{~m}$ system has $25 \mathrm{X}$ the collecting area. Note that the secondary strut obstruction is ignored for this first order analysis.

For a segmented system, some level of mirror actuation will be required for segment phasing (rigid body) and possibly figure control. There are several issues with processing a set of matching segments that will be phased and used as a large primary mirror:

- Radius of Curvature Matching - matching the radius of Curvature (RoC) in gravity to one part in several thousand poses a metrology problem that is likely past the current state-of-the-art for mirror testing. If it can be measured, it can be ion figured into the mirror, but the metrology

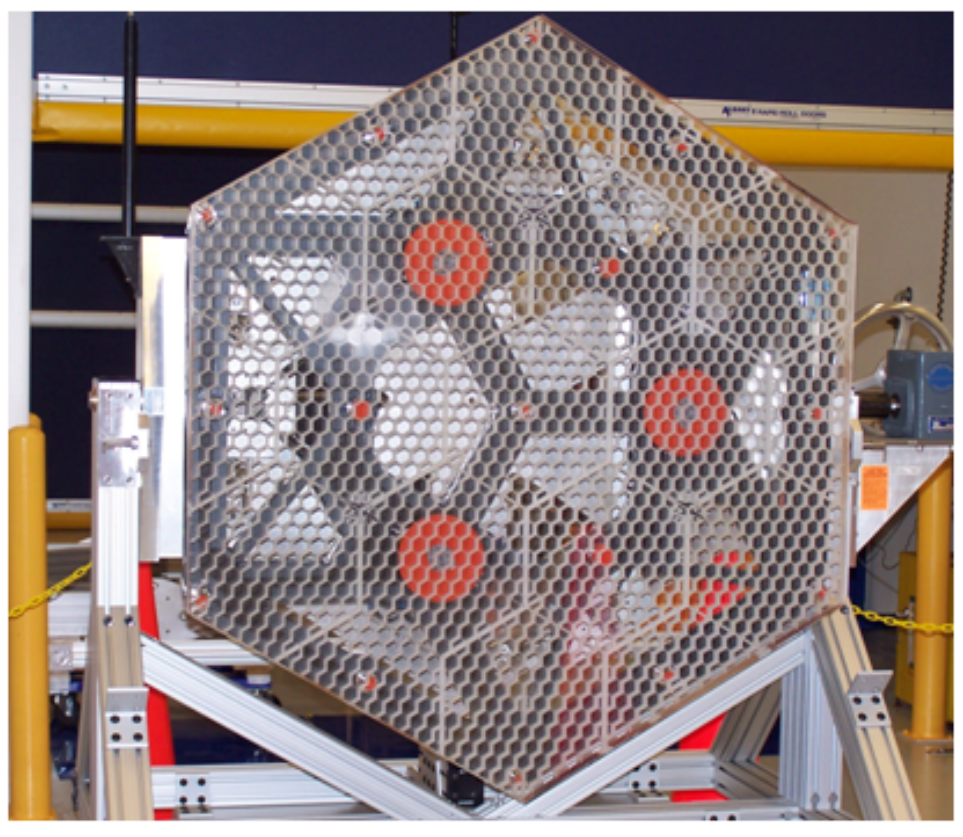

Figure 6.3.3 - A $10 \mathrm{~kg} / \mathrm{m}^{2}$ mirror with a front and back face sheet separated by a lightweight core. error is very difficult. On a $10 \mathrm{~m}$ system, the RoC of the primary mirror is likely to be close to $20 \mathrm{~m}$. Matching that RoC to say 100 microns is an error of 5 parts per million.

- Metrology Errors and Mounting Errors - these errors will likely be driven by astigmatism. And given that this is the first mode of a plate, small errors in mounting strains will manifest themselves as mainly astigmatism. Small errors in placement of the segment will also drive segment level astigmatism and coma.

- On-orbit Thermal Changes - thermal changes tend to be low order. A pure front to back gradient will cause a RoC change. Other thermal gradients will likely impose trefoil (three points) or astigmatism. 
All of these will degrade the performance of the optical system. But it also points to the fact that a large number of actuators are not required for a stiff mirror with a front and back faceplate (Figure 6.3.3). Unlike a dense bed of actuators on a thin face sheet, these stiffer mirrors resist the mid-spatial frequency errors that occur with thin face sheet actuation. As can be seen in Figure 6.3.4, a small number of force actuators, potentially 10 or so, are more than enough to drive in global, low spatial frequency error corrections.

As a demonstration, Figure 6.3.4 shows the influence function for two actuators on the mirror shown in Figure 6.3.2. These influence functions can then be analytically combined to produce many low order error corrections with limited mid-spatial frequency error creation.

The other advantage of the force based system with sparse actuators is that the system has graceful degradation if there is an actuator failure. Some loss in correctability is lost at the $20 \%$ level or so.

Figure 6.3.5 shows how the lightweight test mirror can be corrected from $15 \mathrm{~nm}$ RMS wavefront to around 8nm RMS

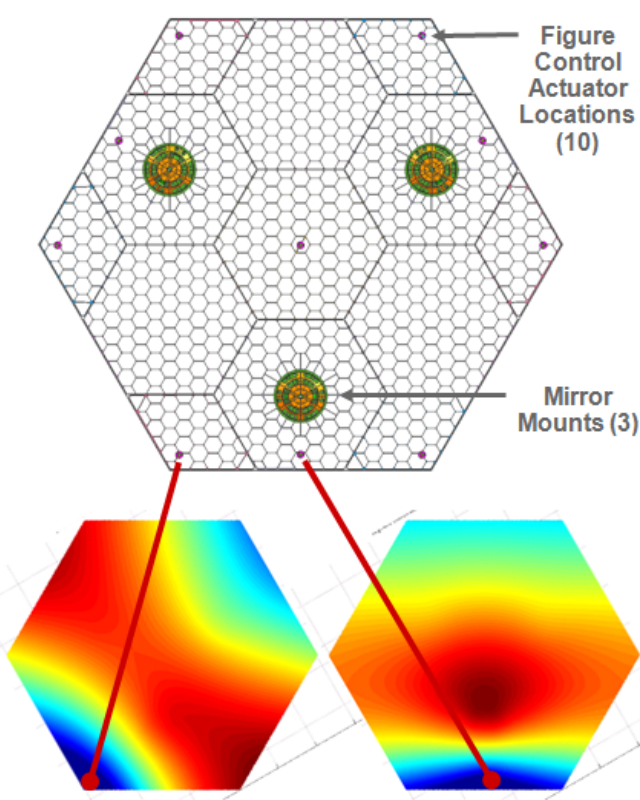

Typical Shape Functions

Figure 6.3.4 - Force actuator influence functions are shown for two of the 10 actuators. wavefront.

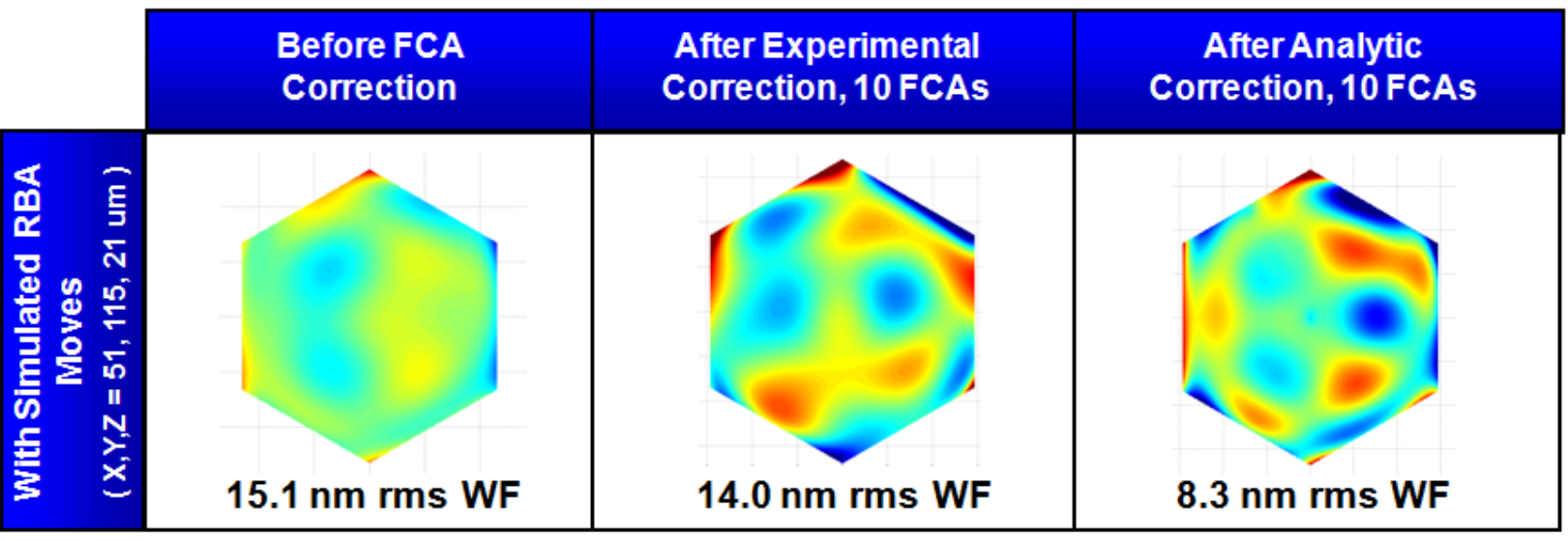

Figure 6.3.5 - Correction of a lightweight mirror for 8nm RMS. Note that the color scales are different in each picture.

Finally, it should be kept in mind that any mirror actuation should be kept to a minimum. The idea of making a low quality mirror and then actuating it to UV/Visible quality will likely result in the creation of an unacceptable amount of mid and high spatial frequency errors. Correction values must be kept to a minimum so that only small on-orbit errors need to be corrected. In fact, potentially only rigid body correction should be considered to enhance the overall stability of the primary mirror. This would require very stiff segments with the addition of segment level precision thermal control to create a very stable primary mirror system. 
As we begin to plan for a very large UV/Visible telescope that will most likely contain a coronagraph as a primary scientific instrument, mirror quality and stability will be crucial performance aspects of the telescope. Even beyond the 10 picometer stability required by the coronagraph, the overall primary mirror quality will still need to be extremely good in order to provide the scientific return required. On a segment basis, it is reasonable to assume that the mirror will need to be finished to $\sim 5 \mathrm{~nm}$ rms surface quality. If you break this down into various errors by spatial frequency, it might look like the budget below:

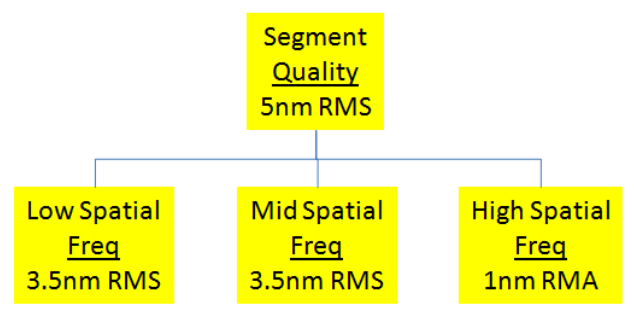

Once the primary mirror is assembled, additional error sources would increase the total error of the system. These are typically low spatial frequency errors caused by telescope structural changes and operational thermal environments which result in primary wavefront errors.

The purpose of this white paper is not to provide an error budget for the primary mirror, but more to provide an assessment of where the state-of-the-art is for producing lightweight segments that would be applicable to a segmented UV/Visible optical system.

There are several choices for mirror materials that have a high TRL status. There are metals or metallic like materials such as Beryllium and $\mathrm{SiC}$ of various configurations. In the glass and glass ceramics, there is Zerodur, ClearCeram ${ }^{\circledR}-Z$, ULE ${ }^{\circledR}$, and Fused Silica.

One clear advantage of the glass and glass ceramics is the ability to ion beam figure (IBF) to create the final high quality surface figure required for this mission. With a PVD (Physical Vapor Deposition) or CVD (Chemical Vapor Deposition) overcoat, the SiC mirrors can also use the IBF process, but this is an extra process which adds to the complexity of the mirror.

The downside of the metallic materials such as $\mathrm{SiC}$ is that they have a $\mathrm{CTE}$ that is 100 times that of the glass/glass ceramics which would drive mirror figure error. The argument is that since $\mathrm{SiC}$ has 100 times the conductivity the mirror will always have a uniform temperature and minimal front to back thermal gradients which drive mirror figure changes. The issue is that transients will still cause a much larger wavefront error even with the higher conductivity than the glass materials. This will drive thermal control parameters to be very difficult. In essence, a near zero CTE makes sense for a UV/Visible optical system. A rigorous trade assessment regarding material selection needs to be conducted to insure that all aspects of material selection (thermal, dynamic, and operational performance and constraints) are considered.

In 2010, Harris completed a program called the Multi Mirror System Demonstrator (MMSD) shown in Figure 6.3.3. In this program, $1.4 \mathrm{~m}$ hexagonal mirror blanks were manufactured in a production environment. Harris demonstrated that $10 \mathrm{~kg} / \mathrm{m}^{2}$ mirror blanks could be fabricated on 3 week centers using existing capability. 


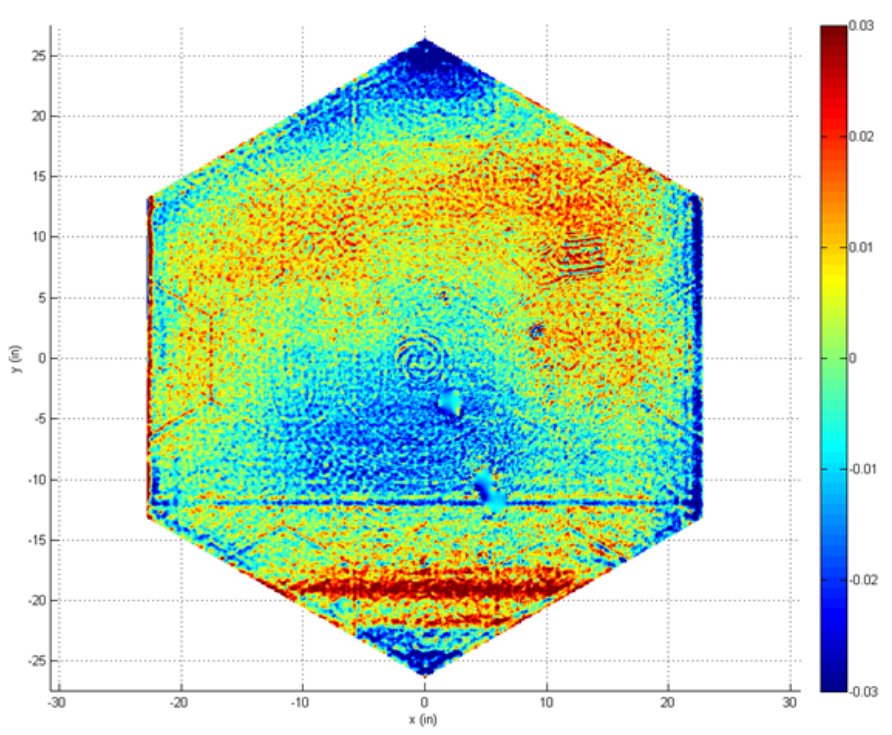

Figure 6.3.6 - MMSD Final wavefront error of 16.3nm RMS.

The mirrors were then processed and used for qualification testing (random vibration and shock). One of the mirrors was finished to the specified final wave front error quality of $19 \mathrm{~nm}$ RMS wave front (9.5nm RMS surface) specification. The MMSD mirror was finished to a $15.1 \mathrm{~nm}$ RMS WFE (7.5nm RMS surface) quality (Figure 6.3.6). It should be noted that there was no PSD type specification levied on the program so the mid spatial content is likely higher than would be acceptable for a dedicated UV/Visible mission. However, IBF could easily be used to address mid spatial frequency errors for mirrors of this type of construction.

Even though the mirror figure quality is approaching the $5 \mathrm{~nm}$ rms notional requirement discussed earlier, there is additional development work before this type of mirror would be applicable to a true UV mission. There is still some core print through which needs to be evaluated against the requirements for this new mission. The other issue will be edges. As can be seen in Figure 6.3.6, the edges need to be treated such that there will be minimum edge relief or potentially no edge relief. These are areas for further development work. Finally, there will need to be metrology measurement development to create a repeatable test to enable very high quality segments to be reliably manufactured.

On the infrastructure side, TMT will provide an excellent precursor for metrology of off-axis segments. This same type of technology may be able to be used on this strategic mission.

In summary, the ability to process a very lightweight segment is close to the high level requirements potentially required. Some level of technology development will fully demonstrate production capability to the LUVOIR specification.

\subsection{Alternate Architectures for Future Astronomy Missions - Ronald S Polidan and the Evolvable Space Telescope and Rotating Synthetic Aperture Teams}

In our presentation to the COPAG we discussed the analyses we have conducted of two alternative architectures for future large astronomical observatories: An Evolvable Space Telescope (EST) concept that starts with a modest size $(4 \times 12$ meter, off axis equivalent to a $\sim 7$-meter telescope collecting area) and evolves to a 16 to 20 meter aperture observatory through assembly and servicing in space and a Rotating Synthetic Aperture (RSA) concept that utilizes a rotating large rectangular aperture as its primary mirror.

A primary goal of this effort is to demonstrate to NASA and the science community the absolute need to explore alternate architectures to mitigate costs, adapt to new technologies, and better integrate into future in-space assembly and servicing. The space observatory environment has changed substantially over the past decades: both positively, such as advances in mirror and wavefront control technologies (e.g. Stahl et al 2014) and a growing in-space operations capability; and negatively, as budgets have 
been greatly reduced. The big question is how we can use the significant investments in technology over the past 10 to 15 years, to create a larger aperture, more productive space telescope at less cost. The alternate architecture approaches discussed in this paper are very viable and apply to all of the future science goals. A culture change will be required to implement one of these alternate architectures, but we believe we should not constrain ourselves to a future of rebuilding our grandfather's space telescope.

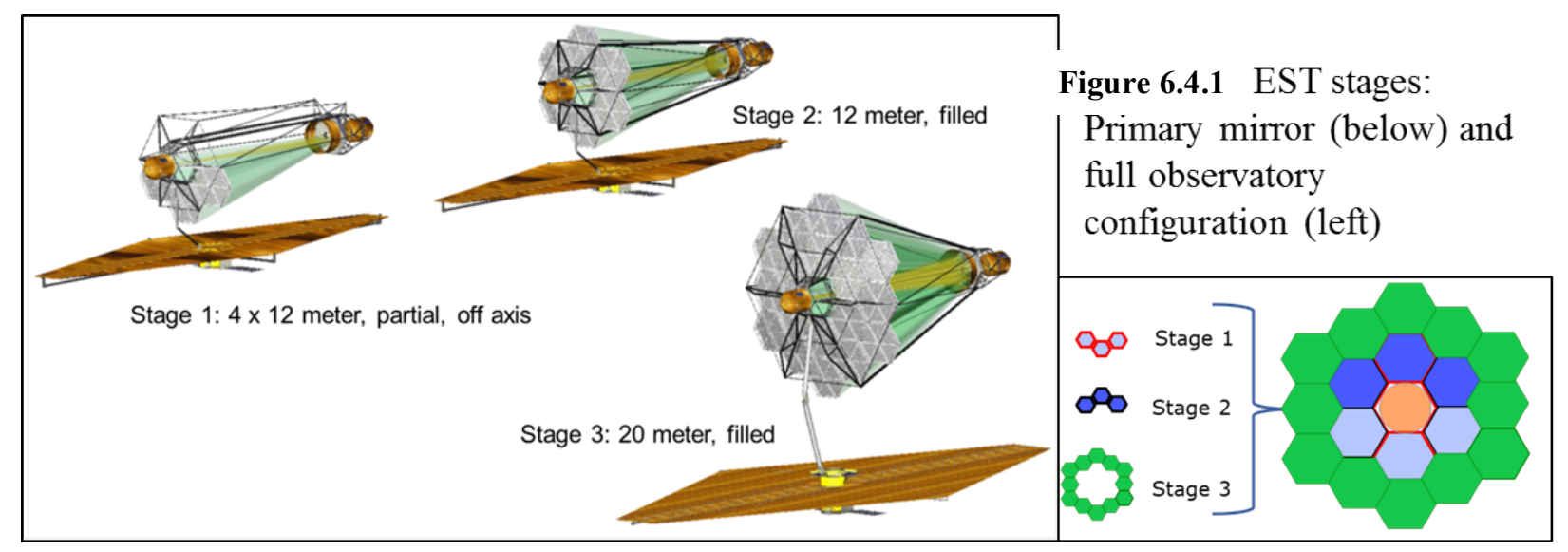

\subsubsection{Evolvable Space Telescope (EST)}

The EST concept focuses upon how to build a large space telescope in a flat science budget era by mitigating big cost peaks, and by encouraging and embracing the development of in-space assembly and servicing infrastructure. EST starts with a modest size off-axis telescope (equivalent to a $\sim 7$-meter filled aperture telescope) that is launched as a fully functional telescope with instruments (EST Stage 1) performing first rank science. After the passage of time ( $\sim 5$ years) an augmentation mission sends additional mirror segments, instruments, and any other hardware to the observatory to grow it, in space,

into a $~ 12$-meter filled aperture observatory (EST Stage 2). Future augmentation missions would again increase its size and add new instruments and the support hardware to create a $\sim 20$-meter filled aperture observatory (EST Stage 3). In short, EST is building upon the Hubble Space Telescope (HST) heritage of service and upgrading to support a space telescope for a long lifetime while increasing its capabilities in accordance with new science issues and advancing technologies. After EST Stage 3 additional augmentations are also possible either to maintain or upgrade the 20-meter telescope for decades or to grow it to even larger sizes with added mirror elements. Other upgrades, such as cryogenic systems to enable coverage of the longer wavelengths available to the James Webb Space Telescope (JWST), may be added to these later stages if the technology advances sufficiently.

\subsubsection{Rotating Synthetic Aperture (RSA)}

The RSA concept offers a very different alternate architecture. This concept was developed for nonastronomical applications and has had a substantial amount of technical investment. Since the technology is relatively mature, our focus has been on analyzing its use for astrophysics, rather than developing a technical solution. RSA is a rectangular aperture telescope that rotates to fill the UVplane. It offers astrophysics a lower cost, greater resolution, better stability, approach that is also able to meet the starlight suppression needs for exoplanet astrophysics. An assessment of the image quality expected from RSA is in progress. 
A full description of both EST and RSA along with additional information on the needed in space infrastructure and a concept for precursor testbed (MoDEST: Modular Demonstration of an Evolvable Space Telescope) on the International Space Station can be found in Polidan et al 2015.

\begin{tabular}{|c|c|c|c|c|c|c|c|}
\hline \multirow[t]{3}{*}{$\begin{array}{l}\text { RSA } \\
\text { Rotating } \\
\text { Synthetic } \\
\text { Aperture }\end{array}$} & \multicolumn{7}{|c|}{$\begin{array}{l}\text { Figure 6.4.2 RSA Observatory configuration (left) and representative } \\
\text { physical parameters and performance comparison to } 12 \text { meter } \\
\text { circular aperture (below) }\end{array}$} \\
\hline & \multicolumn{4}{|c|}{ Rotating Synthetic Aperture Parameters } & \multicolumn{2}{|c|}{$\begin{array}{l}\text { 12-meter Aperture } \\
\text { Comparison }\end{array}$} & \multirow{2}{*}{$\begin{array}{l}\text { Diameter of a } \\
\text { filled aperture } \\
\text { with equivalent } \\
\text { light gathering } \\
\text { capability (m) }\end{array}$} \\
\hline & $\begin{array}{c}\text { Mirror } \\
\text { Aspect } \\
\text { Ratio }\end{array}$ & $\begin{array}{l}\text { Mirror } \\
\text { Length } \\
(\mathrm{m})\end{array}$ & $\begin{array}{l}\text { Mirror } \\
\text { Width } \\
(\mathrm{m})\end{array}$ & $\begin{array}{l}\text { Mirror } \\
\text { area } \\
\left(\mathrm{m}^{2}\right)\end{array}$ & $\begin{array}{l}\text { RSA Fill } \\
\text { Factor }\end{array}$ & $\begin{array}{l}\text { RSA } \\
\text { Integration } \\
\text { Time factor }\end{array}$ & \\
\hline $\begin{array}{l}\text { Mirror technology available today } \\
\text { Substantial USG investment completed }\end{array}$ & $4: 1$ & 16 & 4 & 64 & $56.6 \%$ & 3.12 & 9.03 \\
\hline Raytheon (partnership) has & $6: 1$ & 18 & 3 & 54 & $47.7 \%$ & 4.39 & 8.29 \\
\hline $\begin{array}{l}\text { demonstrated significant elements } \\
\text { Excellent angular resolution }\end{array}$ & $8: 1$ & 20 & 2.5 & 50 & $44.2 \%$ & 5.12 & 7.98 \\
\hline - Requires longer integration times & 9:1 & 32 & 3.5 & 113 & $100 \%$ & 1 & 12 \\
\hline
\end{tabular}

\subsubsection{Summary}

We encourage NASA and the astrophysics community not to be constrained to only traditional space telescope approaches, but rather to fully explore and evaluate alternate architectures for these space telescopes. The technologies that enable these different approaches are either mature or maturing rapidly, so the risk levels are very manageable. The development of in-space infrastructure for spacecraft assembly and servicing is also being actively pursued by many government and industrial organizations, which bodes well for the availability of the needed capabilities in the mid-2030's timeframe. These alternate approaches can offer cost savings and performance enhancements over traditional methods and can enable a more capable astrophysical observatory earlier in time than the traditional approach. They do, however, require a culture change.

\subsection{Far UV Mirror Coating Technology Advances - Juan Larruquert}

\subsubsection{Broadband mirrors}

By far the most efficient broadband mirror coating extending to the FUV is a layer of Al protected with $\mathrm{MgF}_{2}$. It has a reflectance of $\sim 80 \%$ or more all the way from $120 \mathrm{~nm}$ to the infrared and more. It was developed about 50 years ago and it has been basically unsurpassed, except for small reflectance enhancement due to cleaner vacuum deposition systems. It has been largely used in space instrumentation. When high reflectance is required down to $\sim 100 \mathrm{~nm}$, another classical coating is available: Al protected with $\mathrm{LiF}$. The latter material being hygroscopic results in that this coating is only used when there is a real need to reach wavelengths below $120 \mathrm{~nm}$.

A significant reflectance increase both for $\mathrm{Al} / \mathrm{MgF}_{2}$ and for $\mathrm{Al} / \mathrm{LiF}$ in the short $\mathrm{FUV}$ has been recently reported (Quijada et al. 2014). It consists in depositing Al normally and initially protect it with a thin film of $\mathrm{MgF}_{2}$ or $\mathrm{LiF}$, both $\mathrm{Al}$ and fluoride deposited at room temperature (as for standard $\mathrm{Al} / \mathrm{MgF}_{2}$ coatings); then the mirror is heated to $220-250{ }^{\circ} \mathrm{C}$ to complete the $\mathrm{MgF}_{2}$ or LiF film thickness. This results in a reflectance above $90 \%$ at $120-130 \mathrm{~nm}$. This deposition procedure might not be easily applicable for instance on a large telescope mirror for the difficulty to uniformly heat a large-size substrate. Long-term ageing data, and a better knowledge of the coating structure may still be required 
for space applications. A possible replacement for $\mathrm{MgF}_{2}$ may be $\mathrm{AlF}_{3}$, since both have a similar transparency cutoff wavelength; even though no large reflectance differences can be predicted over the two materials, other properties like stability, porosity or roughness might vary over them.

The aforementioned fluorides are deposited by evaporation techniques for highest transparency; this results in films with large porosity, where water and contaminant molecules are embedded in the film. A prospective technique to deposit compact layers of fluorides is Atomic Layer Deposition (ALD). ALD's property of growing a film monolayer by monolayer has suggested that an Al film could be just protected with an ultrathin $\mathrm{MgF}_{2}$ film of few monolayers. This way, not only coating reflectance could increase to almost bare Al reflectance, but its operation range could be extended down to $\sim 83 \mathrm{~nm}$. As another prospective technique, a patent was filed on a procedure to transform the native aluminium oxide grown on an $\mathrm{Al}$ film with a conversion of aluminum oxide into aluminum fluoride by proper flow of fluorine or a fluorine-containing gas in the chamber. These methods to protect Al with an ultrathin fluoride film are under investigation; if they are demonstrated to be operative, they would revolutionize FUV coatings. We need to be cautious on the expectations for these techniques because of the high reactivity of $\mathrm{Al}$ in presence of oxidizing agents, like water vapour; any oxide on Al would strongly degrade Al FUV reflectance.
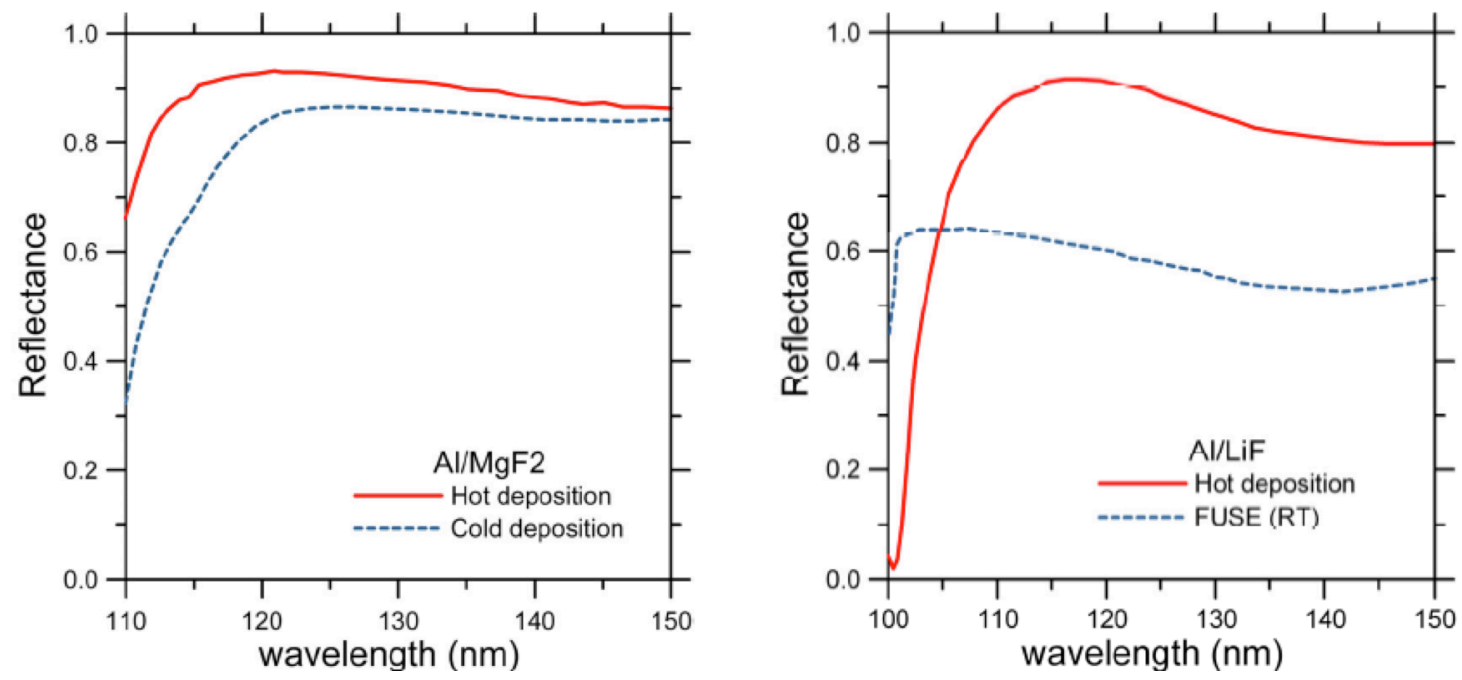

Figure 6.5.1 - FUV reflectance of $\mathrm{Al}+\mathrm{MgF}_{2}$ and $\mathrm{Al}+\mathrm{LiF}$ mirror coatings. $\mathrm{Al}$ and an overcoat of 4-5 (8) nm layer of $\mathrm{MgF}_{2}(\mathrm{LiF})$ deposited at room temperature, later heated to $220^{\circ} \mathrm{C}\left(250^{\circ} \mathrm{C}\right)$ to complete the suitable $\mathrm{MgF} 2(\mathrm{LiF})$ film thickness (Quijada 2014). For comparison, measurements on coatings at room temperature are also displayed.

Below $\sim 100 \mathrm{~nm}$, reflectance is much smaller than above, since there is no transparent material such as to protect an Al film. CVD SiC (Choyke et al. 1977) provides a reflectance of $\sim 40-50 \%$ down to $80 \mathrm{~nm}$; CVD deposition is a high temperature process that requires careful polishing. To deposit at room temperature we can use, single layers of SiC (Keski-Kuha et al. 1988) or B4C (Blumenstock and KeskiKuha 1994), with a reflectance of $\sim 30 \%$ in the spectral range down to $\sim 70-80 \mathrm{~nm}$. A reflectance increase can be obtained with an $\mathrm{Al} / \mathrm{MgF}_{2} / \mathrm{SiC}$ or $\mathrm{Al} / \mathrm{MgF}_{2} / \mathrm{B}_{4} \mathrm{C}$ coating (Larruquert and Keski-Kuha 1999).

\subsubsection{Narrowband Coatings}

Narrowband transmittance filters based on $\mathrm{Al} / \mathrm{MgF}_{2}$ multilayers are another classical coating, which have been used in space instrumentation. They can be designed to peak at any wavelength down to 120 $\mathrm{nm}$. Their main drawback is that peak transmittance is relatively low. 
Reflective multilayer coatings based on two dielectrics have higher peak performance but lower out-ofband rejection than $\mathrm{Al} / \mathrm{MgF}_{2}$ transmittance filters. The main material combinations are $\left(\mathrm{MgF}_{2}\right.$ or $\left.\mathrm{AlF}_{3}\right) /\left(\mathrm{LaF}_{3}\right.$ or $\left.\mathrm{GdF}_{3}\right)$. Narrowband mirrors based on these materials have been obtained for a wavelength as short as $121 \mathrm{~nm}$ (Rodríguez de Marcos et al. 2015) and 135-140 nm (Zukic and Torr 1992; Gatto et al. 2002).

For still shorter wavelengths, a narrowband reflective coating peaked at $\sim 100 \mathrm{~nm}$ based on an Al-LiFSiC-LiF multilayer has been developed (Rodríguez-de Marcos 2013). Its band is suitable to image $\mathrm{H}$ Lyman $\beta(102.6 \mathrm{~nm})$ and O VI lines (103.2-103.8 nm) and to reject longer wavelengths like H Lyman $\alpha$ $(121.6 \mathrm{~nm})$.
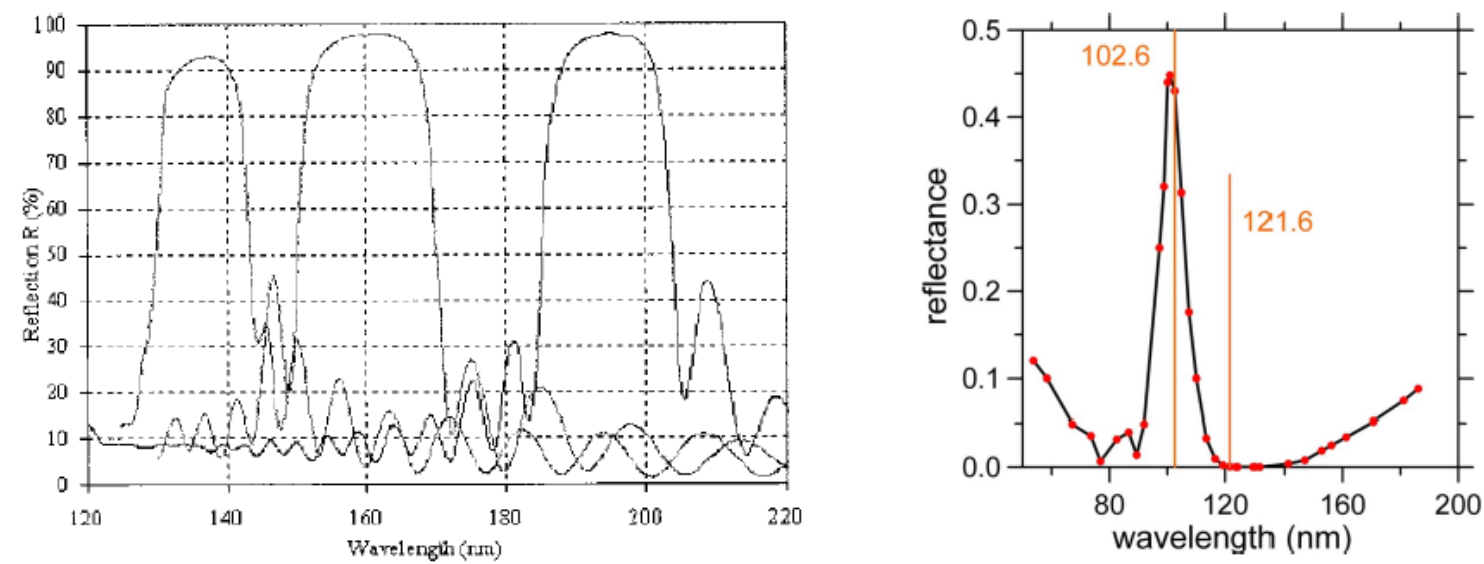

Left: FUV narrowband multilayers (Gatto, Appl. Opt. 41, 3236, 2002). Right: Al-LiF-SiC-LiF narrowband coating peaked at $\sim 100 \mathrm{~nm}$ (Rodríguez-de Marcos, Proc. SPIE 8777, 87771E, 2013)

\section{Missions}

\subsection{Summary of Presentation and Discussion: Probes and non-Flagships - David R. Ardila}

As part of the work for the SIG \#2, the Subcommittee on Mission Diversity discussed mission platforms other than Flagships. The goals were to understand the institutional landscape, and the role of non-Flagships in the context of Flagship plans. Non-flagship missions include Suborbitals (Rockets and Balloons), Cubesats, Missions of Opportunity, Explorers (Small and Medium), and Probes.

We had contributions from Martin Barstow and Ana Gomez de Castro, on the status of European UV efforts, John Hutchings, on Canadian efforts, Mario Perez, on the NASA perspective, and Charles Lillie, on the suborbital experience. Those contributions are summarized elsewhere. The meeting also included wide-ranging discussions on funding and mission cadence.

It was agreed that the augmentation of the Explorer program as recommended by the Decadal Committee has had a strong positive impact on the health of the field. The stable cadence of mission opportunities, not only at the Explorer level, but also at smaller price caps, has contributed to maintain a diverse mission portfolio. Most non-Flagship missions are developed and launch relatively quickly, ensuring knowledge preservation and updated technology. 
The smallest missions contribute crucially to the advancement of NASA's goals and are fundamental to train the next generation of space scientists. Sounding rockets, balloons, and now cubesats represent the foundation in which other space missions are built. In particular, cubesats remain underexploited from the point of view of astrophysics, and can be used for timedomain observations in the UV.

International co llaboration remains very important for the health of the smaller missions, although it is unclear whether or not it results in cost reductions to any of the partners. Europe and Canada are in a situation analogous to the US regarding the UV: no immediate mission prospects,

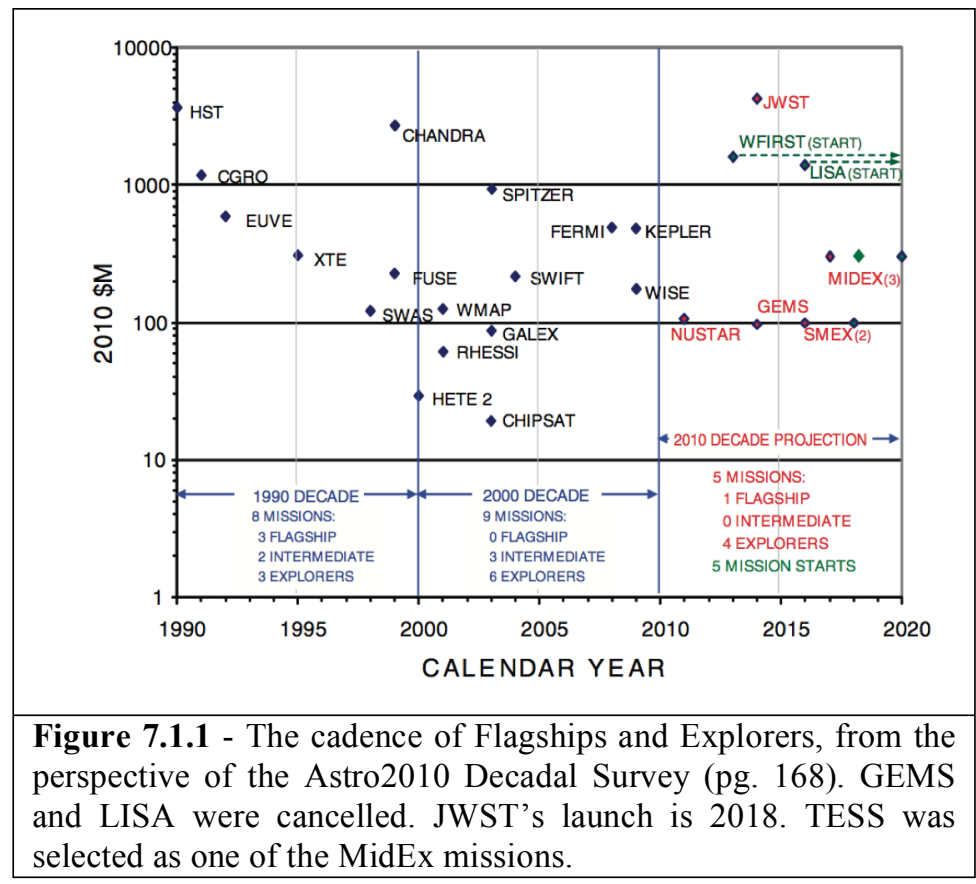
but considerable interest in new missions. Arago, a UV-Vis mission to perform spectropolarimetry, was submitted to ESA in response to the M5 call. M-class missions are $€ 500 \mathrm{M}$ in cost. If approved, it will launch in the 2030 's.

The Subcommittee enthusiastically supported the need to foster public-private partnerships between NASA and private foundations or Universities, in order to reduce costs for the Agency. These

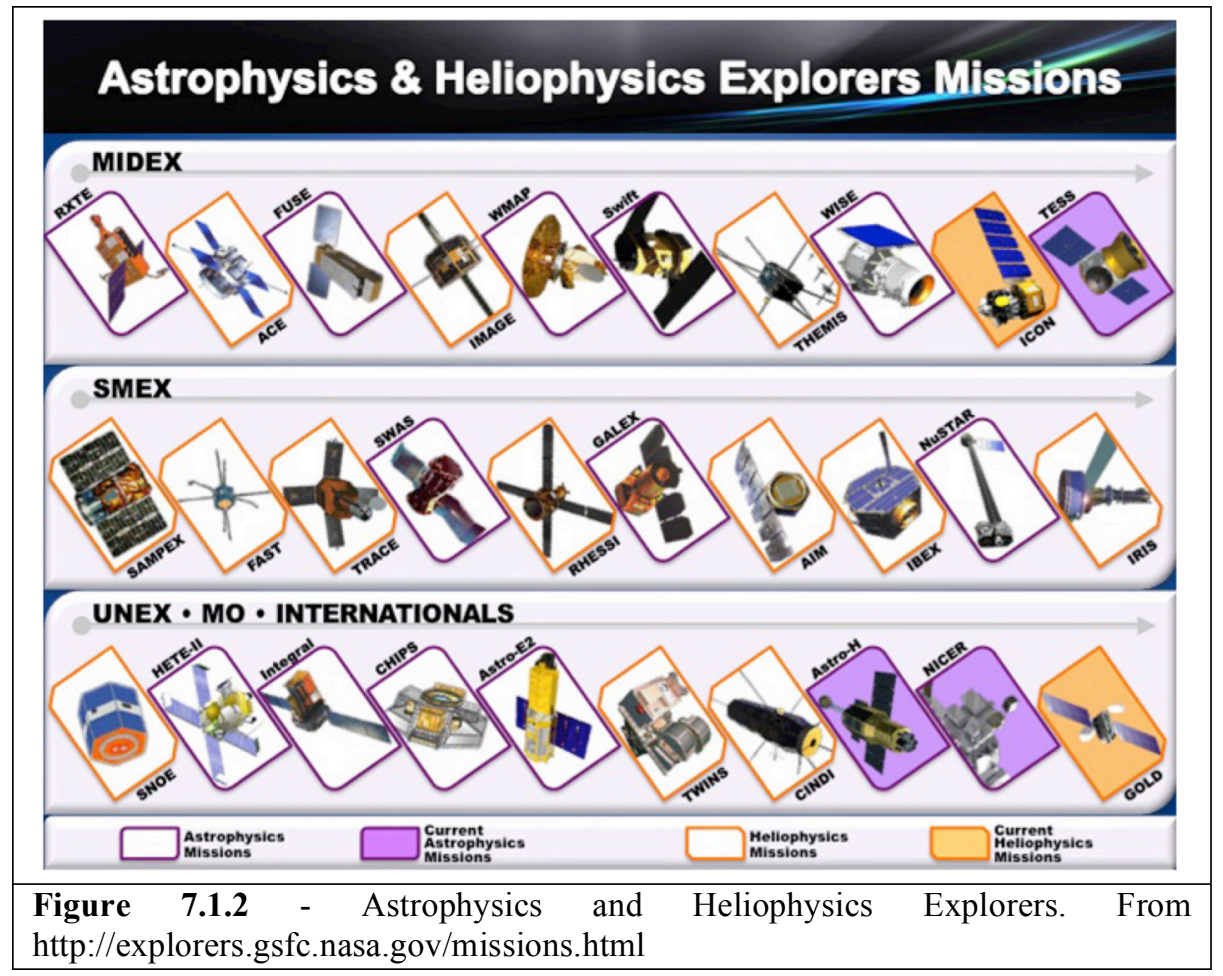

partnerships are common for ground-based telescopes but have yet to be implemented in space missions.

Both at the subcommittee and at the larger SIG level there was strong support for the creation of a Probe line, in the $\$ 1 \mathrm{~B}$ range. However, there was significant concern as to the consequences of a Probe-class line in the cadence of Explorer and smaller missions. A Probe would cost the same as $\sim 3$ MidEx-es. MidEx AOs currently have a 4 year cadence. Furthermore, the Subcommittee agreed that if Probes are going to become part of NASA's portfolio, 
they need to be developed and launched quickly, within the span of $\sim 5$ years.

\subsection{UV Astronomy in Europe - Ana I. Gómez de Castro and Martin A. Barstow}

\subsubsection{The Network for Ultraviolet Astronomy}

There is a broad interest in Europe both in UV science and instrumentation. The community is coordinated through the Network for Ultraviolet Astronomy (NUVA, www.nuva.eu) which created a road map for European UV astronomy/technology in 2008 and continues to coordinate community activity (for instance through the organization of the NUVA workshops every three years). A UV astronomy series has been produced from the workshops and are usually published as special volumes of the Journal: Astrophysics and Space Science (the Springer Verlag professional journal in the field). The publications have been widely distributed and include contributions not only from European but also world-wide activities. Figure 7.2.1 shows the active European members of the NUVA by country.

The community scientific interests cover all possible areas of astrophysics: planetary atmospheres and aurorae, minor bodies in the solar system, exoplanets, star formation and young planetary disks, stellar activity, stellar winds, stellar atmospheres, white dwarfs research, massive stars, stellar populations, interstellar medium (from turbulence or the Local Bubble physics to planetary nebulae and supernovae remnants), interacting binaries and cataclysmic variables, Novae and SNe, star formation at galactic scales and the history of star formation in the Universe at $z<2$, chemical evolution, intergalactic medium, gravitational lensing and astrochemistry. However, the stellar astrophysics (from young stars to cataclysmic variables) community dominates NUVA. Main contributors to all these fields can be found in the NUVA publication series. In 2008, the NUVA submitted its road map for UV astronomy to ASTRONET $^{30}$ : exploit HST, collaborate in the WSO-UV Russian-led mission and prepare for a NASA led 8$10 \mathrm{~m}$ class UV mission.

Recently, NUVA activity has been moved into the new IAU working group of UV astronomy within Division B

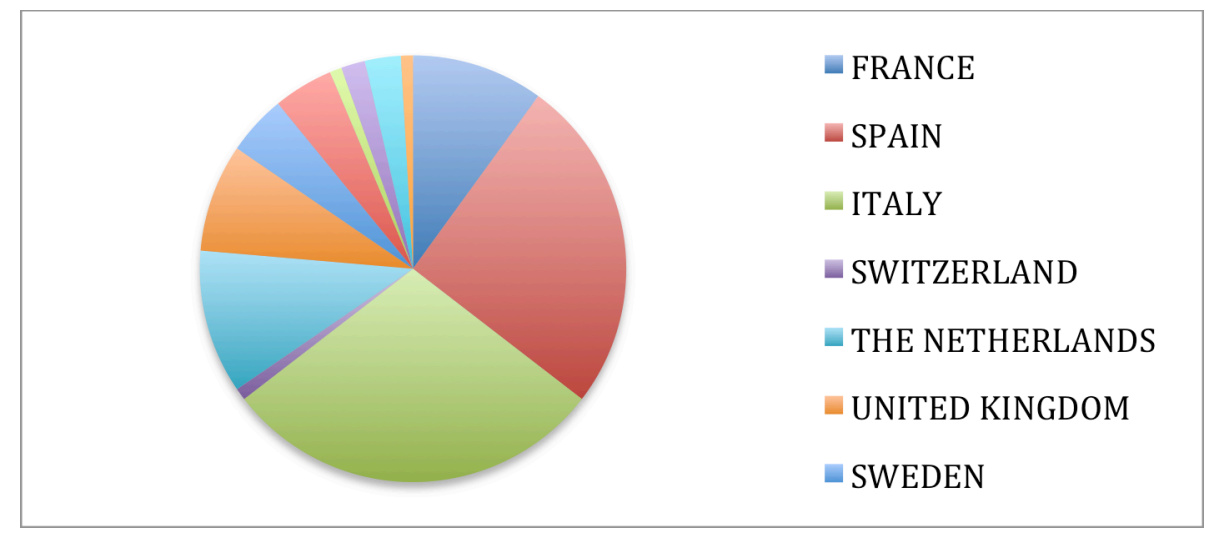

Figure 7.2.1 - Distribution of active NUVA members per European country. (Facilities, Technologies and Data Science) to develop a road map for UV astronomy on a global scale that can exploit the synergies between all interested agencies and academia to coordinate the development of UV mission at all funding levels (from mini, cubesats, to Moon based telescopes or large coordinated missions).

\footnotetext{
${ }^{30}$ ASTRONET is the panEuropean Network elaborating the European Astronomy Infrastructures Road Map through the coordination of the National bodies.
} 


\subsubsection{The European Space Agency}

The European Space Agency (ESA), currently has 20 member states (AT, BE, CH, CZ, DE, DK, ES, FI, FR, IT, GR, IE, LU, NL, NO, PT, PL, RO, SE, UK) and is likely to continue to grow. There are international cooperation agreements with Estonia, Slovenia, Hungary, Cyprus, Latvia, Lithuania, Malta and the Slovak Republic, while negotiations are underway with Bulgaria and Croatia. Canada also takes part in some ESA programmes under a long-standing arrangement.

The ESA main science programme is a mandatory one, in that all countries contribute through their annual subscription to the agency. The value of the contributions is calculated as a proportion of the national GDP of each partner. The main objectives of the programme are to:

- Provide best space tools possible for scientific community to achieve \& sustain excellence, leading the world with discoveries and innovation.

- Choice of projects by scientific excellence

- Selection by competition in a bottom-up process (peer review)

- Stability for scientific research teams

- Reference science framework for the community, national agencies and international partners

- Contribute to sustainability of space capabilities \& infrastructures in Europe

- Provide continuity to industry

- Foster technological innovation

- Attract bright minds to space activities

- Provide perspective to launch services and operations

The programme is driven by scientific excellence through a bottom up process where the scientific community provides broad input through open calls for proposals and competitive peer review. For each mission selected, ESA funds the spacecraft, launch, spacecraft operation and part of the science operations. Member states fund the payloads and the remaining science operations. Therefore, the missions are always partnerships between ESA, a range of member states and institutions within them.

While the community proposes science ideas, ESA provides a strategic framework within which these can be developed, to address broad global themes. These sit under the overarching titles of Horizon 2020 (for missions to fly an operate up to this date) and Horizon 2020+, into the future.

- What are the conditions for planetary formation \& the emergence of life?

- How does the Solar System work?

- What are the physical fundamental laws of the Universe?

- How did the Universe originate and what is it made of?

ESA currently has four mission types, Large (L), Medium (M), Small (S) and Opportunity (O). The large missions are $1 \mathrm{~B} €$ European flagships, with a high innovation level, with a launch every 7-8 years. There may be non-enabling contributions from international partners. Medium missions use current cutting-edge technology and have a $\sim 500 \mathrm{M} €$ cost cap, with a flight every 3-4 years. They can have substantial international contributions, or can be a contribution to a mission of another agency. Small missions are a new, still experimental, element of the ESA programme. They are intended to be fast in execution with a $50 \mathrm{M} €$ cost cap, with the aim of increasing flight opportunities for European scientists. 
Missions of opportunity are contributions to the missions of other agencies. There are several missions currently approved for flight and others under consideration for the ESA Cosmic Vision programme:

- The approved missions in the Cosmic Vision programme, in order of launch date

○ Solar Orbiter (M1) - study of the SUN

- CHEOPS (S1) - transits from known exo-planets

- Euclid (M2) - search for dark energy

- The missions that have been selected, but have not yet received final approval are:

○ JUICE (L1) - Jupiter \& its icy moons

- PLATO (M3) - search for exo-planets down to Earth masses

- Athena (L2) - X-ray observatory

- SMILE (S2) - Earth's magnetosphere and Solar wind

- The Science theme has been selected for L3

○ Gravitational wave mission

There is potential for future involvement in UV missions through forthcoming calls. A European UV Observatory concept was submitted to the $L$ theme selection process. We recognised there was a low likelihood of success but it was a useful placeholder to keep UV in the ESA consciousness. Strong interest in UV remains present in Europe. A series of UV missions have been proposed to ESA, although none have yet been successful.

\subsubsection{Prospective UV Missions}

In chronological order, the current European mission interests are outlined below:

The WSO-UV mission (Shustov et al. 2014; Sachkov et al. 2016) is a Russian led mission to build and operate a $170 \mathrm{~cm}$ primary space telescope that will be orbiting at HEO (geosynchronous) with intended launch in 2021. WSO-UV will work only in the UV (1150-3150 A) and will be provided with instrumentation for high dispersion spectroscopy $(55,000)$ in the full wavelength range, long-slit low dispersion $(1,200)$ spectroscopy also in the full range and imaging capabilities: high angular resolution $(<100$ mas) imaging in the 1150-1750 $\AA$ range and moderate angular resolution $(0.3$ arcsec $)$, wide field of view imaging in the 2000-6000 $\AA$ range. The instruments will be equipped with new technology $\mathrm{CCD}$ detectors develop by $\mathrm{e} 2 \mathrm{~V}$ for the project that will provide a unique sensitivity and dynamic range if lab expectations are fulfilled. Instrument integration will start in 2018.

Spain is a partner of ROSCOSMOS in the project, contributes to the development of the imaging instrument and the software for science operations.

WSO-UV science will be managed from two operations centers located in the Institute of Astronomy of the Russian Academy of Sciences, Moscow and in the campus of the Universidad Complutense de Madrid. WSO-UV will have a core science program and guaranteed time for the countries funding the project. Moreover, there will be open time for the world-wide science community.

The Fresnel Interferometer Array (Koechlin et al. 2009) is a French led proposal to carry out high angular resolution, high dynamic range imaging suitable to image exoplanets and resolve structures to scales of 0.01 mas in its optimal configuration. The optical design is being developed at the Toulouse University/Mid Pyrinees Observatory. An image is formed by diffraction (instead of the common reflection or refraction) in a two spacecraft, formation flying space telescope. One of the spacecraft deploys the screen diffracting the radiation, and another the optics. The proposal was submitted to an 
ESA call for medium size missions in 2009 but was rejected because of its low TRL. The concept has been successfully tested in the visible range on the ground using the Nice telescope, and in the near UV (air UV) in the lab. A prototype is under study to be installed on the International Space Station to reach high TRL in the far UV.

The EUVO proposal (Gómez de Castro et al. 2014) was submitted by the NUVA community in answer to the call issued in March 2013 by the European Space Agency (ESA) for white papers and science drivers for ESA Cosmic Vision program. EUVO was a proposal to build a large ultraviolet-visible observatory (EUVO) to address unexplored areas of the Cosmic Vision program. The consortium behind the EUVO proposal raised the concern about the feasibility of addressing key issues in the Cosmic Vision program such as the investigation of Planets and the search for Life, the chemical evolution of the Universe or the interaction between galaxies and intergalactic medium, without a large UV facility to observe diffuse matter in space. In a subsequent article, a list of requirements was produced for such a facility.

ARAGO, SIRIUS and MESSIER are proposals submitted by the European Astronomy Community to ESA for subsequent calls.

The ARAGO (Neiner et al. 2014) mission is intended to carry out spectropolarimetry in the 119$888 \mathrm{~nm}$ with dispersion between 25,000 in the far UV to 35,000 in the red end to measure the magnetic fields of stars. The mission will be submitted to ESA call for M5; the diameter of the primary mirror is $130 \mathrm{~cm}$.

SIRIUS is an S-class mission aimed at exploring stellar environments and the interactions between stellar sources in the nearby interstellar medium. It is a highly efficient normal incidence R 5000 EUV spectrograph where focusing and dispersion are deliver by a single optical element, a figure multilayer coated diffraction grating. It will observe the sky in two spectral bands covering 18-22nm and 19.5$25 \mathrm{~nm}$.

The MESSIER satellite is an S-class mission designed to explore the extremely low surface brightness universe at UV and optical wavelengths. The two driving science cases target the mildly and highly non-linear regimes of structure formation to test two key predictions of the $\Lambda \mathrm{CDM}$ scenario. The satellite is proposed to drift scan the entire sky in 6 bands covering the 200-1000 nm wavelength range to reach the unprecedented surface brightness levels of $34 \mathrm{mag} / \operatorname{arcsec}^{2}$ in the optical and 37 $\mathrm{mag} / \operatorname{arcsec}^{2}$ in the UV.

Further details of these projects can be found in the NUVA series of publications. Europe's main instrumentation strengths are in detectors (CCDs and MCPs for UV astronomy) and optics (including optical elements and coatings) for UV instrumentation.

\subsubsection{Concluding remarks}

There does seem to be renewed appetite within the ESA hierarchy for involvement in a flagship UVOIR facility to follow HST and JWST, along similar lines to past ESA contributions to these missions. We have set up an ad hoc European committee to promote this idea and future M calls may provide the capacity for ESA contributions. 


\subsection{Astrosat and CASTOR - John Hutchings}

Astrosat is an Indian orbiting observatory launched in Sept 2015, observing from hard X-ray to blue-optical wavelengths. The nominal lifetime is 5 years. There are four co-aligned telescopes and one scanning sky monitor, and all instruments operate simultaneously, allowing for multi-wavelength science. There are two UV telescopes of $\sim 40 \mathrm{~cm}$ aperture (UVIT), with $\sim 1 / 2$ degree field of view and $\sim 1$ " resolution. They provide simultaneous coverage of FUV, NUV, and VIS channels, each with a suite of filters. The UV channels also have gratings that provide $\mathrm{R} \sim 100$ spectra of all objects. Canada is a partner, having provided the UVIT detectors.

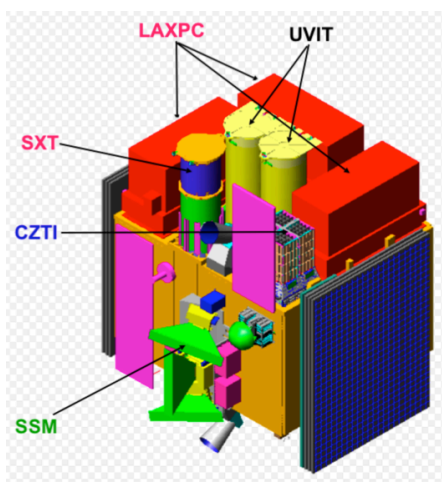

Figure 7.3.1 - Astrosat orbiting observatory

Commissioning is completed as of March 2016, and guaranteed time science is under way until September 2016. Proposal time begins in October 2016, and that will include time open to all after year 2. All instruments are working well. The website is at http://astrosat.iucaa.in/.

CASTOR is a concept for a $1 \mathrm{~m}$-class UV-optical telescope with wide field of view and image quality $\sim 0.15 \%$. A detailed concept study was carried out by the Canadian Space Agency (CSA) and contractors, and has been featured in a number of conferences in recent years.
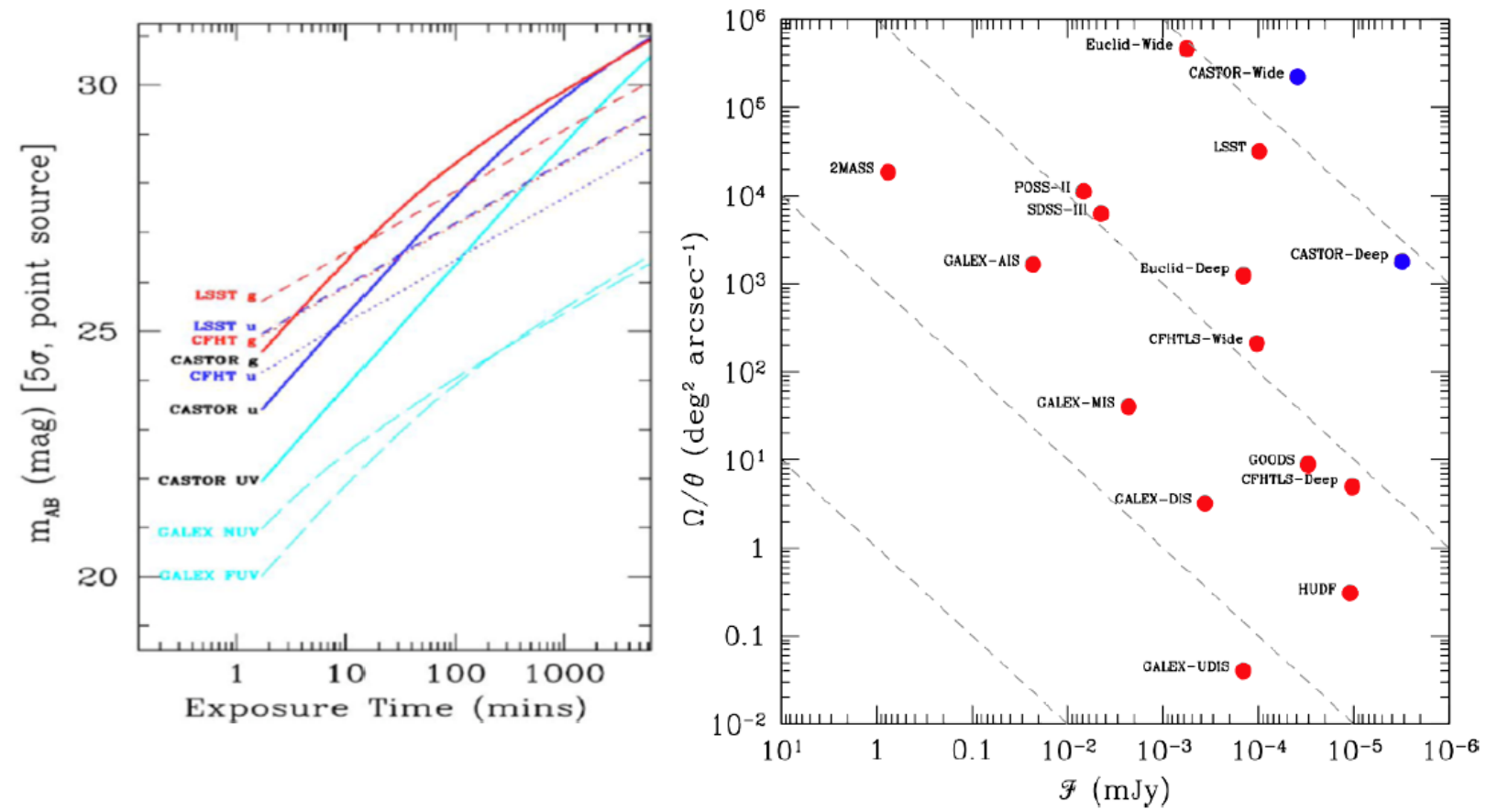

Figure 7.3.2 - Some CASTOR performance metrics for surveys, compared with others.

The concept is a 1-m diameter unobscured Three Mirror Anastigmat telescope providing good image quality over a $1.16^{\circ}$ x $0.58^{\circ}$ field of view. A 725 Megapixel camera with wavelength coverage from $150-550 \mathrm{~nm}$, in three wavelength channels, allows access to wavelengths not visible from the ground. The telescope can perform surveys of an area of 5,000 deg2 in $<2$ years, with planned efficient 
operations. The aperture provides u-band Wide Survey sensitivity of $>27 \mathrm{AB}$ magnitude. There is an option for dispersed imaging with the addition of one moving part.

Subsequent work has developed and prototyped a detector array, and further optimization of the optomechanical layout to provide improved coverage in wavelength bands, and provide the necessary redleak elimination in the UV. A CSA phase 0 and updated science reference study are in preparation.

CASTOR can provide valuable short-wavelength survey coverage for Euclid and WFIRST, as well as LSST. CASTOR will also be a facility for a wide range of PI and legacy science.

Significant partnership opportunities are open for this proposed mission.

\subsection{Suborbital Vehicles - Charles F. Lillie}

\subsubsection{Definition}

NASA's suborbital flight activities involve the use of sounding rockets, aircraft, high-altitude balloons, and suborbital reusable launch vehicles to advance science, and to train the next generation of scientists and engineers who will play a key role in future space missions.

Sub-orbital space vehicles are vehicles that are able to reach space, but are unable

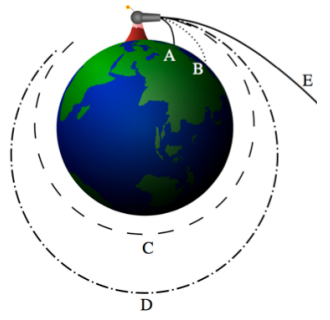

Figure 7.4.1 Trajectories versus burnout velocity to achieve the velocity required to orbit the earth. Thus they have elliptical orbits with an apogee greater than 100 kilometers, a perigee less than $0 \mathrm{~km}$. These vehicles use rocket motors that boost them to velocities between 1.1 and $7.7 \mathrm{~km} / \mathrm{sec}$, and then coast to their peak altitude after engine burnout. Their peak altitude and downrange capability increases with their burnout velocity. Figure 7.4.1 illustrates the elliptical orbits for vehicles with a range of final velocities. Paths A and B depict sub-orbital trajectories; Paths $\mathrm{C}$ and D are orbital trajectories; and path $\mathrm{E}$ is an escape trajectory.

Although NASA's aircraft and high altitude balloons cannot reach space, as defined by the "Von Karman Line" at an attitude of $100-\mathrm{km}$, they also provide an important opportunity to train personnel, develop new instruments, and obtain observations to address cutting-edge space and earth science research questions.

\subsubsection{Purpose}

Suborbital vehicles are an important tool for astronomers who wish to study the universe at wavelengths that are unobservable from the earth's surface. Figure 7.4.2 shows the altitudes to which electromagnetic radiation can

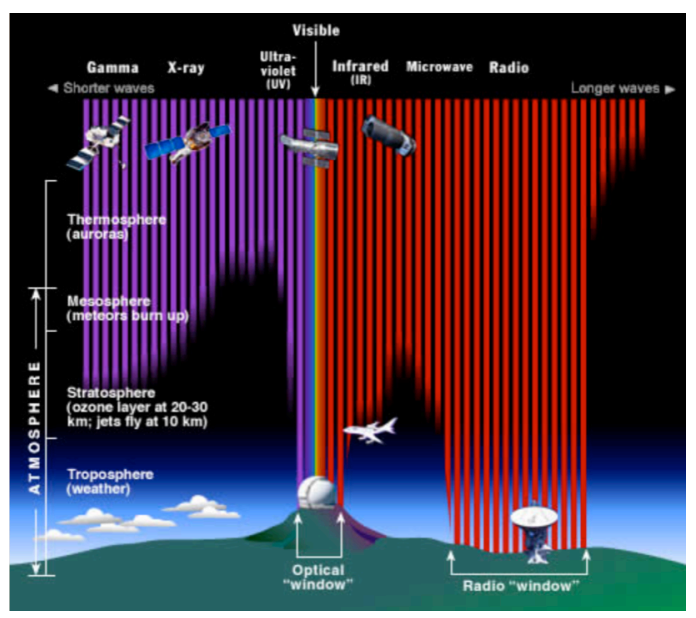

Figure 7.4.2 - Altitude at which electromagnetic radiation is absorbed by the earth's atmosphere penetrate the earth's atmosphere. With the exception of the $\sim 1-\mathrm{cm}$ to $11-\mathrm{m}$ "Radio Window" and the narrow 300-nm to 1100-nm "Optical Window", sub-orbital vehicles and space vehicles such as NASA's Great observatories (Figure 7.4.2) are required to open windows on the universe at wavelengths from the Gamma-ray to long wavelength radio region of the spectrum. 


\subsubsection{Available Vehicles}

NASA's suborbital program utilizes a variety of vehicles for their Suborbital Research Program. The standard scientific Helium-filled balloons can carry payloads of 3,600 kg to an altitude of 42-km and remain there for as long as 2 weeks. And flights of up to 100 days are now possible with NASA's Ultra Long Duration Balloon (ULDB).

NASA also utilizes several aircraft to test new instruments and collect scientific data for Earth science, including calibration data for satellites in earth orbit. They also operate the SOFIA aircraft that can carry its $2.5-\mathrm{m}$ telescope to an altitude of $41,000 \mathrm{ft}$. (well above most of the water vapor in the atmosphere) to observe celestial objects in the 0.3 to 655 micron spectral region.

Rocket-powered aircraft have been used for scientific research in the past, and NASA has plans to do so again when the next generation of such aircraft become available. During the mid-1960s the X-15-1 was modified to carry scientific instruments to an altitude of 250,000 feet. This included the addition of a hatch over the instrument compartment that could be opened after engine burnout, allowing a pointing platform with four cameras to photograph stars in the 180 to $240 \mathrm{~nm}$ spectral region. X-15-1 flew 28 space science experiments, ranging from astronomical observations to micrometeorite collection. NASA is now planning to fly earth and space science experiments on reusable

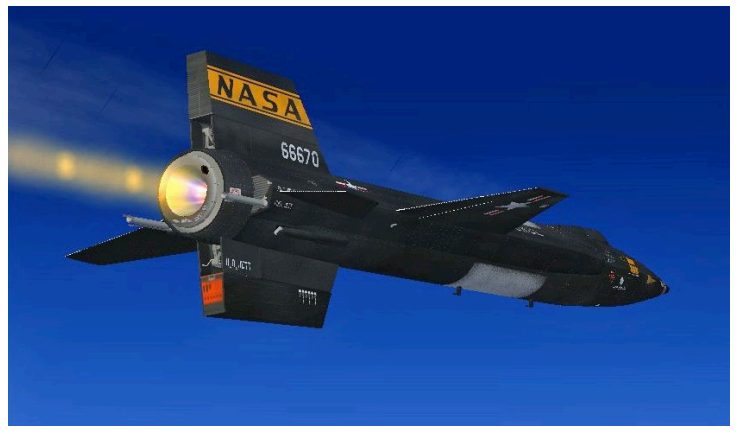

Figure 7.4.3 - X-15-1 accelerating after launch from under the wing of a B-52 at 45,000 ft. and Mach 0.8 suborbital vehicles such as Virgin Galactic's Spaceship-2, and XCOR's Lynx when they become operational. These rocket-powered aircraft will be able to reach altitudes of $110-\mathrm{km}$, well above the edge of space. Unfortunately they will have only 4 or 5 minutes of time for observations above the atmosphere before falling back to earth and gliding to a landing.

Sounding rockets have been the primary suborbital vehicle for scientific research. Since 1959 NASA has launched over 2900 sounding rockets to test instruments to be used on satellites and spacecraft and to provide information about the Sun, stars, galaxies and the Earth's atmosphere and radiation. Today there are 15 different types of sounding rockets ranging in size from the single stage, 2.1-m tall Super Arcas to the four-stage 20-m Black Brandt XII. These rockets carry scientific payloads to altitudes ranging from 48 to more than 1300 kilometers with observation times of 5 to 20 minutes. Figure 7.4.4 shows a 3stage Black Brandt X shortly after launch. The $3^{\text {rd }}$ stage motor is ignited once the vehicle system reaches exo-atmospheric conditions. The standard vehicle is $43.8-\mathrm{cm}$ in diameter with a 3:1 ogive nose shape. The Black Brandt X can lift an $800-\mathrm{lb}(362.9-\mathrm{kg})$ payload to $375-\mathrm{km}$ or a $250-\mathrm{lb}(113.4-\mathrm{kg})$ payload to $875-$

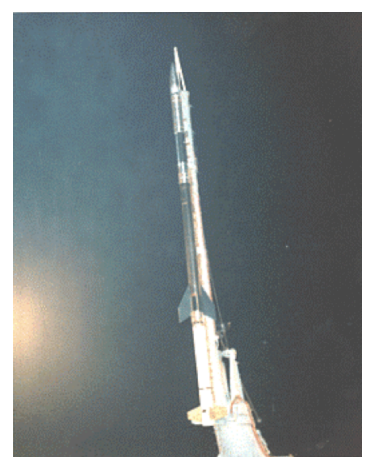

Figure 7.4.4 - Black Brandt X $\mathrm{km}$.

\subsubsection{Advantages of Suborbital Vehicles}

Suborbital vehicles have several advantages for scientific research when compared with spacecraft in orbit: (1) missions are orders of magnitude less expensive; (2) The time from concept to launch (18 to 36 months) is far shorter, allowing graduate students to conduct a mission and obtain the data for a thesis; (3) they enable the maturation and flight demonstration of new instrument technologies, 
including instrument architectures, detectors, optical coatings and gratings, etc.; (4 they conduct focused cutting-edge science investigations that address current scientific questions; (5) they can obtain insitu observations at sub-orbital altitudes; and (6) the payloads can be recovered, allowing them to be modified to correct for in-flight anomalies, updated with new enabling and enhancing technologies, and reused for additional missions to observe other objects.

The downside of course, is the limited observing time in space, i.e., only 5 to 20 minutes for sounding rockets and manned re-useable vehicles, and 5 to $50+$

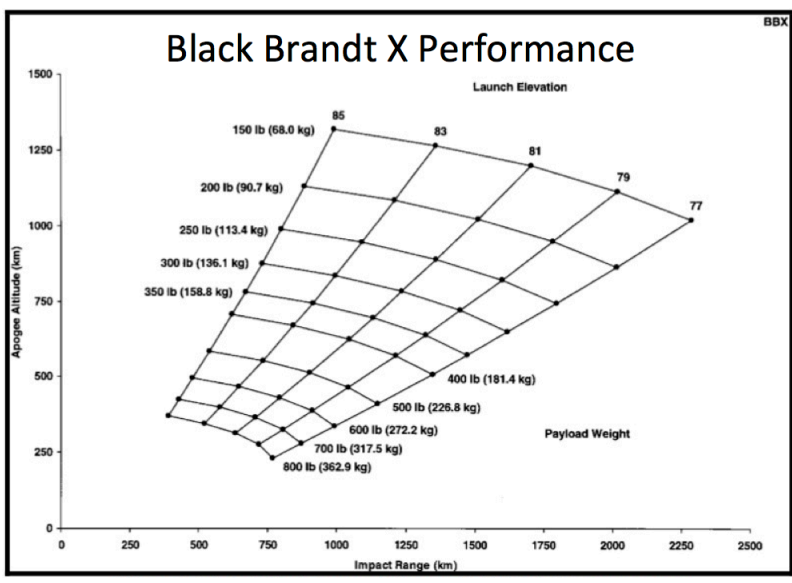

Figure 5. Black Brandt $X$ altitude as a function of payload mass and elevation angle days for high altitude balloons. Nevertheless. Suborbital vehicles are an important, integral part of a balanced research program for NASA that includes small and medium explorers, probes, and large flagship missions.

\section{Recommendations to the COPAG Executive Committee by the Workshop}

\subsection{Background}

In January 2015, Dr. Paul Hertz asked the NASA's Astrophysics Program Analysis Groups (PAGs) to collect and analyze the community's thoughts on which future Flagship missions NASA should give input on for the 2020 NRC Decadal Survey on Astronomy and Astrophysics. This request to the PAGs was motivated by the need to ensure that any Flagship concept presented to the Decadal Survey was sufficiently developed both scientifically and technologically as to be considered plausible and feasible. Four possible Flagship concepts are currently under discussion: a Far-IR Surveyor, a HabitableExoplanet Imaging Mission, a UV/Optical/IR Surveyor, and an X-Ray Surveyor. NASA intends to invest limited funds in key technology development that would enable the science and mission concepts envisioned. To this end, the directed charge was:

- Each PAG should reach out to their communities to review the starting set of four Flagship concepts and to suggest additions, subtractions, and providing useful commentary.

- Each PAG will consider what mission studies should be performed to advance astrophysics as a whole.

- Each PAG should not consider that any one mission concept "belongs" to them.

Reports submitted by the PAGs will be used by NASA as the basis for the following subsequent steps to develop these mission concepts:

- Identify a small set of candidate large mission concepts

- Form a community-based Science and Technology Development Team (STDT) for each of these mission concepts

- Ask the teams to articulate the key science drivers and to identify critical technology studies needed in the interim. 
- Fund studies of critical technology requirements and ask the teams to review these

- Prepare a case for delivery to the 2020 Decadal Survey committee.

In November 2014, the NASA Advisory Council (NAC) Astrophysics Subcommittee approved a request from the Cosmic Origins Program Analysis Group Chair, Dr. Ken Sembach, to establish a new Science Interest Group (SIG \#2) on the future of UV-visible astronomy from space. This SIG met for the first time in January 2015 at the Seattle AAS meeting, and proceeded to solicit community input in direct support of the PAG charges with the specific focus on the UV-visible passband. In June 2015, the SIG held a 2-day workshop at NASA's Goddard Space Flight Center to consider not only the issue of Flagship science, but broader compelling science in the UV-visible, the technology needed to enable that science, and the spectrum of mission sizes needed to conduct that science. As part of that workshop, the assembled community took the opportunity to address the questions levied by Dr. Hertz in a spirited discussion. This summary outlines a set of recommendations to the COPAG Executive Committee motivated by the discussion and agreement arrived at during the workshop.

\subsection{Recommendation Specifics - an Executive Summary}

The main recommendations of this SIG Workshop to the COPAG Executive Committee (EC) are summarized here. More details on the presented science and technology germane to these recommendations are given in the following sections.

- The SIG does not suggest any additions or subtractions to the list of four concept studies.

- The SIG strongly recommends the endorsement and study of both a $10 \mathrm{~m}+$ class UVOIR Surveyor and the smaller UV-visible HabEx mission concepts.

- The SIG recognizes the potential of the $10+\mathrm{m}$ UVOIR Surveyor to make compelling discoveries in both cosmic origins and exoplanet science.

- Based on input from the ExoPAG, the SIG assumes that HabEx concept is smaller than the UVOIR Surveyor although the exact HabEx aperture has not yet been determined. At this time, the SIG did not explicitly explore the astrophysical science applications of a smaller aperture mission. Although a smaller aperture telescope may address many of the same astrophysical themes, it cannot achieve the sensitivity or resolution that a larger $10+\mathrm{m}$ telescope will deliver. Even more than its aperture, the suitability of HabEx for cosmic origins applications depends critically on two yet-to-be defined capabilities: its field of view in the $\mathrm{UV} /$ visible and its sensitivity into the far ultraviolet.

- Among the critical UV-visible technologies that need continued investment to be sufficiently mature for consideration by the 2020 Decadal survey are optical coatings, large format radiation-tolerant high efficiency photon-counting detectors, coronagraphs, and the accommodation of coronagraphic instruments in large-scale telescopes via technologies to address dynamics of the structure. It is particularly important to develop coatings and multiplexing detectors/instruments that maximize sensitivity into the far ultraviolet without compromising coronagraphic requirements.

- The SIG believes that a broad spectrum of precursor missions will be necessary to vet the new science, mature the required technologies and establish the credible workforce required to augment the scientific productivity and impact of large Flagships, while controlling risk and cost. These other missions include not only suborbital and explorer-class facilities, but also Probe-class spacecraft $(\operatorname{cost}<\$ 1 \mathrm{~B})$, more ambitious than Explorers but more focused than Flagships. 
- The SIG also believes there are several compelling opportunities to work with international partners towards Flagship-class missions that fulfill the science goals identified in this document, and we encourage NASA to explore these possibilities. In that regard, we see a benefit to including ESA, CSA and Asia-based scientists as observers in the STDTs and request that NASA pursue this possibility.

\subsection{Compelling Science that Supports a Flagship Mission Implementation}

The SIG and the COPAG solicited input from the astrophysics community in the form of short white papers and workshop presentations. We received a total of $\mathbf{3 5}$ of these relevant to the UV-visible and to Flagship-class implementation. While this document is purposely intended to be brief for easier digestion, we have asked all those contributors to summarize in Science Traceability Matrix (STM) form the goals and requirements of their science. We include this summary matrix below. This information has been collected in an attempt to represent the breadth of science that a Flagship-class UV-visible mission could address.

This STM details the range of science, the types of capability and how that science maps into fundamental mission properties such as aperture, spectral range, throughput, image scale, spectroscopic resolution, and other factors. On occasion our discussions included valuable relevant IR science goals and technologies. Many of the science problems presented here are motivated by the SIG's great interest in a $10 \mathrm{~m}+$ class telescope and require apertures of this size. We did not explore the extent to which 2030's era science goals could be partially addressed by smaller apertures. However, it is clear that wide-field imaging and high sensitivity into the Far-UV are essential requirements regardless of aperture size.

The scientific identity of each STM submission has been preserved to enable the STDT to interact with each submitter as appropriate. We could have further integrated the submissions to similar science questions and capabilities, but that would have represented additional work that NASA HQ has advised it did not want the PAGs to engage in to prepare these recommendations. We have merely presented work already completed as part of the SIG's deliberations running up to our workshop and provide it to the COPAG EC as information to be considered.

We have deliberately not tried to repeat the work of the AURA report "From Cosmic Birth to Living Earths", released July 6, 2015, which did an excellent job of summarizing many kinds of science that could be done with a Surveyor Flagship-class mission facility. However, we recognize that the AURA report was not complete due to their space limitations, and we wanted to make sure that no corner of the community was left unrepresented. That said, our summary is also not complete, but we believe it is more representative of the full range of potential UV-visible Flagship science.

This work should also be viewed in tandem with an earlier call from the COPAG about the future of UV-visible astrophysics in 2012 (Scowen et al 2014), where the focus was more centered on Hubbleclass or slightly larger missions, and also in light of the findings of the Theia study (Kasdin 2009) which laid out the astrophysics goals for a 4m-class UVOIR mission.

\subsection{Technologies that Need Investment}

During the deliberations for the SIG workshop it became clear that technological advances for the UVvisible passband have been proceeding through a variety of development investments by both NASA's 
Science Mission Directorate and Space Technology Mission Directorate. However, the low maturity level of some crucial technologies does give cause for concern and the SIG makes the strong recommendation that additional immediate investment be provided if those technologies are to be advanced enough for consideration by the Decadal Survey in 2020. The SIG expresses some concern that the timescale for the STDT process is not necessarily consistent with the pressing schedule needed to ensure that the required technologies will conform with SMD guidelines for readiness.

The state of those technologies, their impact, and what additional investments could yield, are summarized in the attached table. Specific conclusions and recommendations include:

- Reflective Mirror Coatings: development of a reflective coating that can be deployed in a relevant environment (i.e., mirrors for space missions) that improve upon $\mathrm{MgF}_{2}$ over $\mathrm{Al}$. In addition, the coating must be scalable to a large aperture. The goals would be a reflectivity of $>$ $70 \%$ from $90-120 \mathrm{~nm}$, a reflectivity of $>90 \%$ from $120 \mathrm{~nm}$ to 1.7 microns, uniformity $<1 \%$ for wavelengths $>90 \mathrm{~nm}$, and polarization $<1 \%$ over the bandpass

- UV Detectors: visible/NIR detectors are excellent devices with improvements mostly being incremental for cosmic origins science, unless exoplanet science is required. One key feature would be better radiation tolerance than is available to state of the art silicon detectors. The lifetime of an instrument using these visible/NIR detectors is limited by the detector, not the spacecraft. For the UV, improvements to DQE - greater than $70 \%$ at $90-120 \mathrm{~nm}$, larger formats $(>4 \mathrm{k} \times 4 \mathrm{k}$ resolution elements), and improvements in dynamic range would increase the science capabilities of a flagship mission. A stable $(<1$ pixel) wavelength solution and the ability to observe to a very high signal to noise $(>100)$ is critical to select scientific programs. UV detectors also need to be photon-counting in order to take advantage of the UV minimum in the natural sky background.

- Opto-mechnical design and validation of large optical systems: demonstrate fabrication of thermally-stable mirrors within a production schedule that have $<7 \mathrm{~nm}$ RMS surface-figure. Demonstrate alignment and phasing of segments with gravity release and modeling to demonstrate on-orbit capability. Develop thermally- and dynamically-stable structures for mirror and instrument support. Demonstrate vibration isolation, metrology, and actuator performance to required levels. Validate structural-thermal-optical performance (STOP) models to the picometer level, and verify testing and on-orbit stability of the optical system. Additionally development of methods to reduce the areal cost of primary monolithic mirrors.

- Polarization-preserving telescope coatings and configurations: develop low polarization reflective coatings that can be deployed in a relevant environment (i.e., mirrors for space missions). In addition, the coating must be scalable to a large aperture. The goal would be a polarization uniformity of $0.01 \%$ to enable space-based precision polarimetry and coronagraph contrasts as high as $1 \mathrm{E}-11$ necessary for terrestrial exoplanet characterization.

After good discussions with our exoplanet science colleagues, we recognize that any Flagship-class UV-visible mission will involve a partnership between the astrophysics and exoplanet communities and that it is essential that technology studies include the accommodation of both kinds of science.

The SIG recommends to the COPAG EC that additional investment be made in the listed critical technologies to enable the next generation science listed in the included Science Traceability Matrix. 
Scowen et al.: Finding the UV-Visible Path Forward 


\section{References}

Abbott et al. 2016, Phys. Rev. Lett. 116, 061102

Andersson, B-G, Lazarianm A. \& Vaillancourt, J.E., 2015, ARA\&A, 51, 531

Andrews et al. 2013 ApJ, 767, 51

Andrews et al. 2014, ApJ, 793, 4

Arnouts, S., Schiminovich, D., Ilbert, O., et al. 2005, ApJL, 619, L43

(http://adsabs.harvard.edu/abs/2005ApJ...619L..43A)

Belu at al. 2011A\&A...525A..83B

Benjamin A. Mazin, B., Bumble, B., Meeker, S., O’Brien, K., McHugh, S., and Langman, E. "A superconducting focal plane array for ultraviolet, optical, and near-infrared astrophysics," Opt. Express 20, 1503-1511 (2012)

Berdyugina, S.V., et al., 2011, ApJ, 728, 6.

Bertone, S., Aguirre, A., \& Schaye, J. 2013, MNRAS, 430, 3292

Bétrémieux \& Kaltenegger 2013ApJ...772L..31B

Bianchi et al. 2011, Ap\&SS335, 249

Bjorkman, K.S., Bjorkman, J.E. \& Wood, K., 2000, ASPC, 214, 603.

Blandford et al. 2010, Nat Acad. Press

Blumenstock and Keski-Kuha, Appl. Opt. 33, 5962, 1994

Borthakur, S. Heckman, T., Tumlinson, J., Bordoloi, R., Kauffmann, G., Catinella, B., Schiminovich, D., \& Davé, R. 2016, ApJ, in press (arXiv:1609.06308)

Botticella et al. 2012, A\&A, 537, A132

Bouret et al. 2015, MNRAS, 449, 1545

Bouwens, R. J., Illingworth, G. D., Blakeslee, J. P., \& Franx, M. 2006, ApJ, 653, 53

(http://adsabs.harvard.edu/abs/2006ApJ...653...53B)

Brammer, G.B. et al. 2011ApJ...739...24B, 2011

Brown, T.M., et al. 2008, ApJ, 685, L121

Calzetti et al. 2010, ApJ, 719, L158

Chen, H.-W., Gauthier, J.-R., Sharon, K., Johnson, S.D., Nair, P., Liang, C.J. 2014, MNRAS, 438, 1435

Choyke et al., Appl. Opt. 16, 2013, 1977

Dahlen et al. 2012, ApJ, 757, 70

Deming et al. 2009PASP..121..952D

des Marais et al. 2002AsBio...2..153D

Domagal-Goldman et al. 2014ApJ...792...90D

Drozdovsky et al. 2008, Galaxies in the Local Volume

Du, X., Shapley, A.E., Martin, C.L., Coil, A.L. 2016, 829, 64

Finkelstein, S. L., Ryan, R. E., Jr., Papovich, C., et al. 2015, ApJ, 810, 71

(http://adsabs.harvard.edu/abs/2015ApJ...810...71F)

Ford, A., et al. 2014, MNRAS, 444, 1260

France et al. 2013ApJ...763..149F

France et al. 2012SPIE.8443E..05F

García et al. 2014, ApJ, 788, 64

Gatto et al., Appl. Opt. 41, 3236, 2002 
Gómez de Castro, A.I., Appourchaux, T. Barstow, M. A. et al. 2014, "Building galaxies, stars, planets and the ingredients for life between the stars. The science behind the European Ultraviolet-Visible Observatory", Astrophysics and Space Science, 354, 229

Gonzalez, V., Labbe, I., Bouwens, R. J., et al. 2010, ApJ, 713, 115 (http://adsabs.harvard.edu/abs/2010ApJ...713..115G)

Grundy et al. 2013, Icarus, 223, Issue 2, pp. 710-713

Hamden, E. T., Greer, F., Schiminovich, D., Nikzad, S., and Martin, D. C., "UV photon-counting CCD detectors that enable the next generation of UV spectroscopy missions: AR coatings that can achieve 80-90\% QE, " SPIE 8453, 845309 (2012)

Hamden, E. T., Jewell, A. D., Gordon, S., Hennessy, J., Hoenk, M. E., Nikzad, S., Schiminovich, D., and Martin, D. C., "High efficiency CCD detectors at UV wavelengths, " SPIE 9144, 91442X (2014)

Heckman, T. M., Sembach, K. R., Meurer, G. R., et al. 2001, ApJ, 558, 56 (http://adsabs.harvard.edu/abs/2001ApJ...558...56H)

Hennessy, J., Jewell, A. D., Hoenk, M. E., and Nikzad, S., "Metal and dielectric filters for solar blind silicon ultraviolet detectors" Applied Optics, 54, 3507 (2015)

Hoadley, K., France, K., Nell, N., Kane, R., Schultz, T., Beasley, M., Green, J., Kulow, J., Kersgaard, E., and Fleming, B., "The assembly, calibration, and preliminary results from the Colorado highresolution Echelle stellar spectrograph (CHESS), " SPIE 9144, 914406 (2014)

Hoang, T., Lazarian, A. \& Martin, P.G., 2014, ApJ, 790, 6.

Horiuchi et al. 2013, ApJ, 769, 113

Hu et al. 2012ApJ...761..166H

Huang, Y.-H., et al. 2016, MNRAS, 455, 1713

Inoue, A. K., \& Iwata, I. 2008, MNRAS, 387, 1681

(http://adsabs.harvard.edu/abs/2008MNRAS.387.1681I)

Joung, M. R., Putman, M. E., Bryan, G. L., Fernandez, X., \& Peek, J. E. G. 2012 ApJ, 759, 137

Kaltenegger et al. 2007ApJ...658..598K

Kasdin, J., "THEIA: Telescope for Habitable Exoplanets and Interstellar/Intergalactic Astronomy", AIP Conf. Proc. 1158, 359 (2009)

Keeney, B., et al. 2013, ApJ, 765, 27

Kerěs, D., Katz, N., Weinberg, D.H., \& Dave, R. 2005, MNRAS, 363, 2

Keski-Kuha et al., Appl. Opt. 27, 2815, 1988

Kishimoto, M, 1999, ApJ, 518, 676

Koechlin, L., Serre, D., Deba, P., et al. 2009; "The fresnel interferometric imager", Experimental Astronomy, 23, 379

Koskinen et al. 2013Icar..226.1678K

Kroupa 2001, ASPC, 228, 187

Labbe, I., Gonzalez, V., Bouwens, R. J., et al. 2010, ApJL, 716, L103

(http://adsabs.harvard.edu/abs/2010ApJ...716L.103L)

Lammer et al. 2014MNRAS.439.3225L

Larruquert and Keski-Kuha, Appl. Opt 38, 1231, 1999

Lehner, N., et al. 2013, ApJ, 770, 138

Lehner, N., Howk, J.C., \& Wakker, B.P 2015, ApJ, 804, 79

Linsky et al. 2014ApJ...780...61L

Lomax, J.R., et al., 2015, A\&A, 573, A43.

Loyd \& France 2014ApJS..211.... 
Madau, P., Haardt, F. \& Rees, M. J. 1999, ApJ, 514, 648

(http://adsabs.harvard.edu/abs/1999ApJ...514..648M)

Madau, P. \& Dickinson, M., 2014ARA\&A..52..415M, 2014

Maíz-Apellániz \& Rubio 2012, A\&A, 541, 54

Martin, P.G., Clayton, G.C., \& Wolff, M.J., 1999, ApJ, 510, 905

McCandliss, S. R., Andersson, B., Bergvall, N., et al. 2012, arXiv:1209.3320

(http://adsabs.harvard.edu/abs/2012arXiv1209.3320M)

McCandliss, S. R., Kruk, J. W., Blair, W. P., et al. 2008, SPIE, 7011, 701120

(http://adsabs.harvard.edu/abs/2008SPIE.7011E..20M)

Meynet et al. 1994, A\&AS, 103, 97

Morin et al., 2008, MNRAS, 390, 567

Murphy et al. 2011, ApJ, 732, 126

Neiner, C., Baade, D., Fullerton, A., et al. 2014, "UVMag: stellar formation, evolution, structure and environment with space UV and visible spectropolarimetry", Astrophysics and Space Science, 354,215

Nelson, D., et al. 2015, MNRAS, 448, 59

Packham, C., Young, S. \& Axon, D. , 2011, ASPC, 449, 405.

Peeples, M.S., et al. 2014, ApJ, 786, 54

Polidan R. S., et al "An evolvable space telescope for future astronomical missions: 2015 Update", Proc. SPIE 9602-6, San Diego, CA, USA, 2015

Quijada et al., Proc. SPIE 8450, 84502H, 2012

Quijada et al., Proc. SPIE 9144, 91444G, 2014

Ricotti, M., Gnedin, N. Y., \& Shull, J. M. 2002, ApJ, 575, 49

(http://adsabs.harvard.edu/abs/2002ApJ...575...49R)

Robertson, B. E., Ellis, R. S., Furlanetto, S. R., \& Dunlop, J. S. 2015, ApJL, 802, L19

(http://adsabs.harvard.edu/abs/2015ApJ...802L..19R)

Rodríguez-de Marcos, Proc. SPIE 8777, 87771E, 2013

Rodríguez-de Marcos et al., Proc. SPIE 9627, 96270B, 2015

Roederer \& Lawler, ApJ, 750, 76, 2012

Rubin, K.H.R., Prochaska, J.X., Koo, D.C., Phillips, A.C., Martin, C.L., \& Winstrom, L.O. 2014, ApJ, 794, 156

Sachkov, M., Shustov, B, Gomez de Castro, A.I., 2016; "World Space Observatory Ultraviolet mission: status 2016", Proc. SPIE 9905, Space Telescopes and Instrumentation 2016: Ultraviolet to Gamma Ray, 990504 (18 July 2016); doi: 10.1117/12.2233085

Scowen, P., Perez, M., Neff, S., Benford, D., "Scientific objectives for UV/visible astrophysics investigations: a summary of responses by the community (2012)", Experimental Astronomy, 37, \#1, pp.11-35 (2014)

Seager et al. 2013ApJ...777...95S

Seager et al. 2009and..book..123S

Segura et al. 2010AsBio..10..751S

Shkolnik \& Barman 2014AJ...148...64S

Shustov, B., Gómez de Castro, Ana I., Sachkov, M. et al., 2014; "WSO-UV progress and expectations", Astrophysics and Space Science, 354, 155

Siegmund, O. H. W., McPhate, J. B., Vallerga, J. V., Tremsin, A. S., Frisch, H. E., Elam, J. W., Mane, A. U., and Wagner, R. G., "Large area event counting detectors with high spatial and temporal resolution, " Journal of Instrumentation 9, C04002 (2014) 
Stahl, P. et al, Proceedings of SPIE, 9143, 91431S, 2014

Stocke, J.T., et al. 2013, ApJ, 763, 148

Thomas, H-C, et al. 2012, A\&A. 546, A104

Tian et al. 2014E\&PSL.385...22T

Tramper, F., Sana, H., de Koter, A., \& Kaper, L. 2011, ApJL, 741, L8

Tremsin, A. S., Hull, J. S., Siegmund, O. H. W., McPhate, J. B., Vallerga, J. V., Dabiran, A. M., Mane, A., and Elam, J., "Opaque gallium nitride photocathodes in UV imaging detectors with microchannel plates, " SPIE 8859, 88590X (2013)

Tumlinson, J., et al. 2011, Science, 334, 948

Tumlinson et al. 2015AAS...22533819T

Vallerga, J., Raffanti, R., Cooney, M., Cumming, H., Varner, G., and Seljak, A., "Cross strip anode readouts for large format, photon counting microchannel plate detectors: developing flight qualified prototypes of the detector and electronics, SPIE 9144, 91443 (2014)

Wang, J., et al., 2011, MNRAS, 412, 1081

Werk, J., et al. 2014, ApJ, 792, 8

Whitmore et al. 2010, AJ, 140, 75

Wiktorowicz, S.J., 2009, ApJ, 696, 1116.

Yan, H. \& Lazarian, A., 2008, ApJ, 677, 1401.

Williams et al. 2011, ApJ, 734, L22

Wotta, C.B., Lehner, N., Howk, J.C., O’Meara, J.M., \& Prochaska, J.X. 2016, ApJ, in press (arXiv:1608.02584)

Zukic and Torr, Appl. Opt. 31, 1588, 1992 


\section{Science Traceability Matrix}

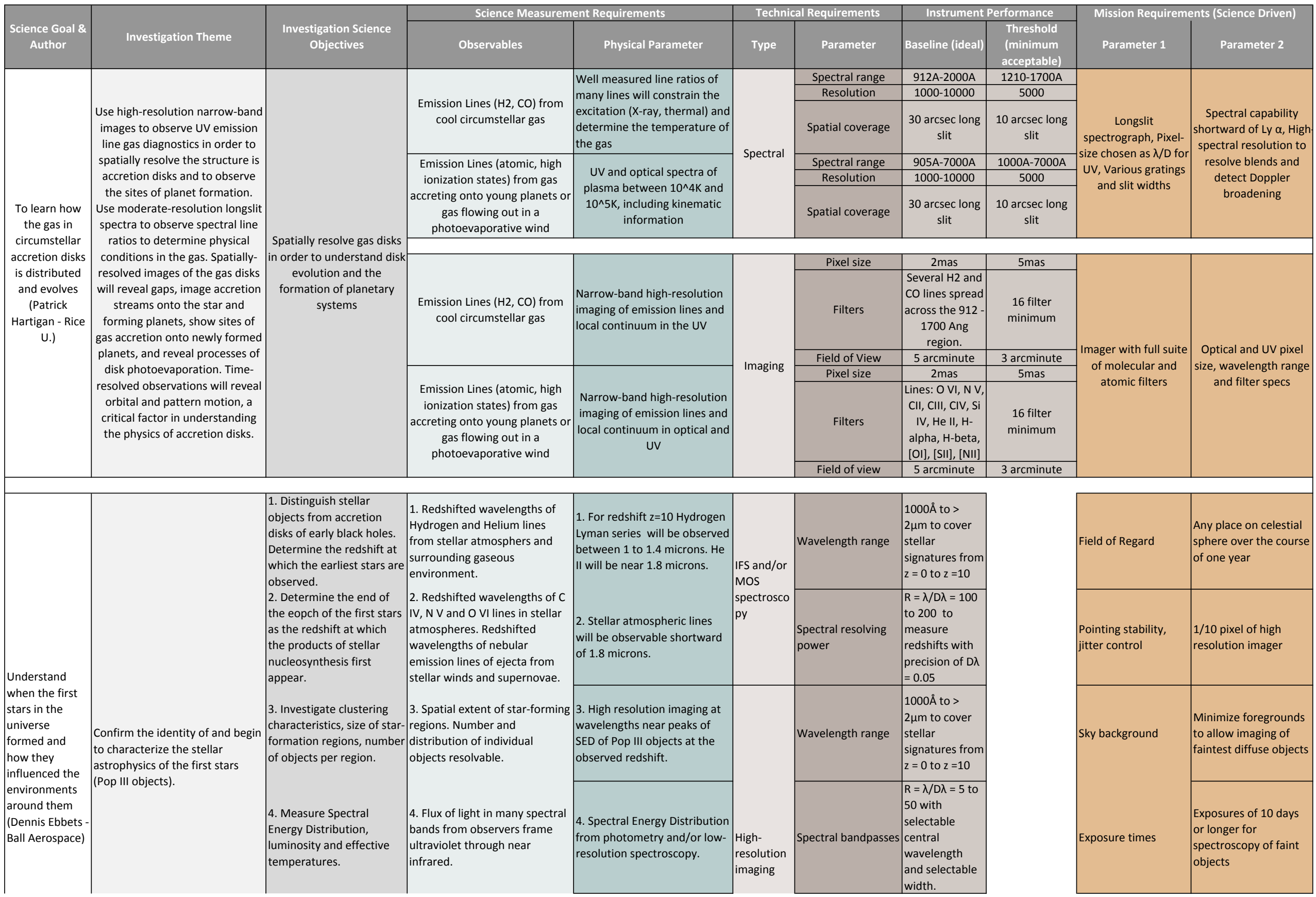




\begin{tabular}{|c|c|c|c|c|c|c|}
\hline & & $\begin{array}{l}\text { 5. Detect and characterize } \\
\text { supernova explosions of } \\
\text { Pop III objects }\end{array}$ & $\begin{array}{l}\text { 5. Rise time, peak magnitude } \\
\text { and decay rate. }\end{array}$ & \begin{tabular}{|l|} 
5. Multi-band light curves with \\
a cadence sufficient to sample \\
rise-time, isolate peak \\
brightness and characterize \\
decay. Low resolution \\
spectroscopy to detect \\
signatures of products of \\
explosive nucleosynthesis.
\end{tabular} & & Spatial Resolution \\
\hline \multirow{15}{*}{$\begin{array}{l}\text { Understand } \\
\text { how the } \\
\text { universe came } \\
\text { to be (mostly) } \\
\text { ionized } \\
\text { (Stephan } \\
\text { McCandliss - } \\
\text { JHU) }\end{array}$} & \multirow{15}{*}{$\begin{array}{l}\text { Determine galaxy and stellar } \\
\text { cluster luminosity functions at } \\
\text { energies above the hydrogen } \\
\text { ionization edge from } 0<z<3 \text {, } \\
\text { covering } 11 \text { Gyrs of evolution }\end{array}$} & \multirow{15}{*}{$\begin{array}{l}\text { Detect and measure the } \\
\text { flux above and below the } 1 \\
\text { Rydberg in the rest frame } \\
\text { of at least } 25 \text { galaxies and } \\
\text { stellar clusters per } \\
\text { redshift bin per luminosity } \\
\text { bin to yield a confidence } \\
\text { level of } 20 \% \text { per bin. }\end{array}$} & \multirow{7}{*}{$\begin{array}{l}\text { Mesure Spectral Energy } \\
\text { Distribution above and below } \\
912(1+z) \text { with SNR }>5 \text { at } \\
900(1+z) \text { from stellar clusters } \\
\text { and galaxies. }\end{array}$} & \multirow[t]{3}{*}{ Intensity } & $\begin{array}{l}\text { Sensitivity } \\
\text { (Expected } \\
\text { limits for } \\
\text { escape } \\
\text { fraction: } \\
100 \% \text { at } \\
\text { low, } 1 \% \\
\text { escape at } \\
\text { high z) } \\
\end{array}$ & $\begin{array}{l}f_{900(1+z)}=10^{-15} \mathrm{erg} \mathrm{cm} \\
2 \mathrm{~s}^{-1} \AA^{-1} \text { at } z=0.02 \\
\mathrm{f}_{900(1+2)}=10^{-20} \mathrm{erg} \mathrm{cm} \\
2 \mathrm{~s}^{-1} \AA^{-1} \text { at } z=3\end{array}$ \\
\hline & & & & & $\begin{array}{l}\text { Backgroun } \\
\text { d }\end{array}$ & $\begin{array}{l}\text { Interplanetary zodi } \\
\text { and Lyman alpha } \\
\text { limited }\end{array}$ \\
\hline & & & & & \begin{tabular}{|l|} 
Dynamic \\
Range \\
(whole \\
sample) \\
\end{tabular} & 100000 \\
\hline & & & & \multirow{4}{*}{ Wavelength (Energy) } & Bandwidth & 900 to $3650 \AA$ \\
\hline & & & & & \begin{tabular}{|l|} 
Spectral \\
Resolution \\
\end{tabular} & $10 \AA$ \\
\hline & & & & & $\begin{array}{l}\text { Redshift } \\
\text { resolution }\end{array}$ & \begin{tabular}{|l|}
$\sim$ \\
1 Gyr bins over 11 \\
Gyrs $(0<z<3)(11$ \\
bins)
\end{tabular} \\
\hline & & & & & $\begin{array}{l}\text { Luminosity } \\
\text { resolution }\end{array}$ & $\begin{array}{l}0.25 \text { magnitude bins } \\
\text { over } 5 \text { magnitudes } \\
\text { of apparent } \\
\text { magnitude ( } 20 \text { bins })\end{array}$ \\
\hline & & & $\begin{array}{l}\text { Hot Stellar Cluster } 30 \text { to } 100 \mathrm{pc} \\
\text { in diameter, Evolution from } \\
0.02<z<3\end{array}$ & \multirow{5}{*}{ Angular } & \multirow{2}{*}{ Resolution } & \begin{tabular}{|l|}
0.075 to 0.250 \\
arcseconds at $z=$ \\
$0.02,0.004$ to 0.013 \\
arcseconds at $z=3$
\end{tabular} \\
\hline & & & \begin{tabular}{|l|} 
Galaxies $1 \mathrm{kpc}$ to $100 \mathrm{kpc}$ in \\
extent, Evolution from $0.02<z<3$
\end{tabular} & & & \begin{tabular}{|l|}
2.50 to 250 \\
arcseconds at \\
$z=0.02$ \\
0.13 to 13 \\
arcseconds at $z=3$
\end{tabular} \\
\hline & & & \multirow{3}{*}{\begin{tabular}{|l|} 
Total angular coverage for \\
galactic luminosity functions $>1$ \\
degree to reduce cosmic \\
variance
\end{tabular}} & & $\begin{array}{l}\text { Instantane } \\
\text { ous FOV }\end{array}$ & $\begin{array}{l}\text { Slits 1.5 } \times 3 \\
\text { arcseconds }^{2}\end{array}$ \\
\hline & & & & & $\begin{array}{l}\text { Pointing, } \\
\text { multiplexin }\end{array}$ & $\begin{array}{l}\text { Multiobject } \\
\text { spectroscopy over }\end{array}$ \\
\hline & & & & & $\mathrm{g}$ & $6 \times 6$ arcminutes $^{2}$ \\
\hline & & & \multirow{3}{*}{$\begin{array}{l}25 \times 20=500 \text { Lyman continuum } \\
\text { leaking objects per luminosity } \\
\text { function. } 11 \text { Luminosity } \\
\text { functions. Total galactic targets } \\
\text { 5500. Total cluster targets } \\
5500 .\end{array}$} & \multirow{3}{*}{ Temporal samples } & $\begin{array}{l}\text { Integration } \\
\text { time }\end{array}$ & $\begin{array}{l}\text { Depends on } \\
\text { Aperture, Detector } \\
\text { and MSA }\end{array}$ \\
\hline & & & & & $\begin{array}{l}\text { Single } \\
\text { observatio } \\
\text { n duration }\end{array}$ & $\begin{array}{l}\text { Several (5) hours for } \\
\text { faintest, few } \\
\text { seconds for } \\
\text { brightest. } \\
\text { Multiplexing is } \\
\text { required. }\end{array}$ \\
\hline & & & & & $\begin{array}{l}\text { Cosmic } \\
\text { Time and } \\
\text { resolution }\end{array}$ & $\begin{array}{l}0 \text { to } 11 \text { billion yrs, } \\
\text { 1Gyr resolution }\end{array}$ \\
\hline
\end{tabular}

\begin{tabular}{|l|l|}
\hline Mission duration & $\begin{array}{l}>5 \text { years to allow } \\
\text { complete temporal } \\
\text { coverage of light } \\
\text { curves of } 10 \text { or more } \\
\text { Pop III supernovae }\end{array}$ \\
\hline
\end{tabular}

Aperture Driver

(likely requires 12

meter Gregorian 2

bounce to meet low

end)

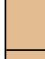

Orbit Driver (L2)

Detector Driver

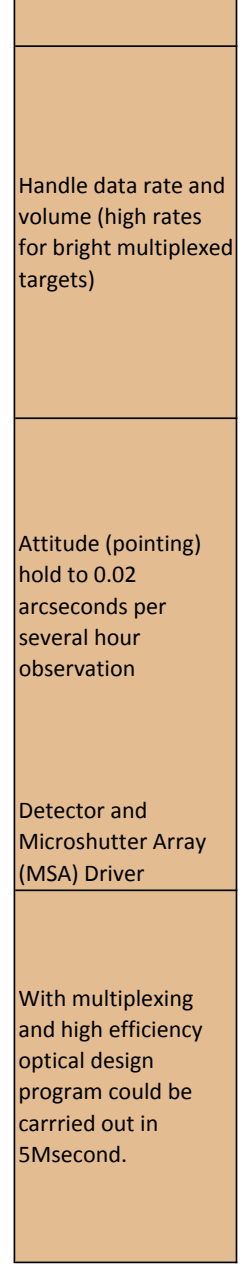









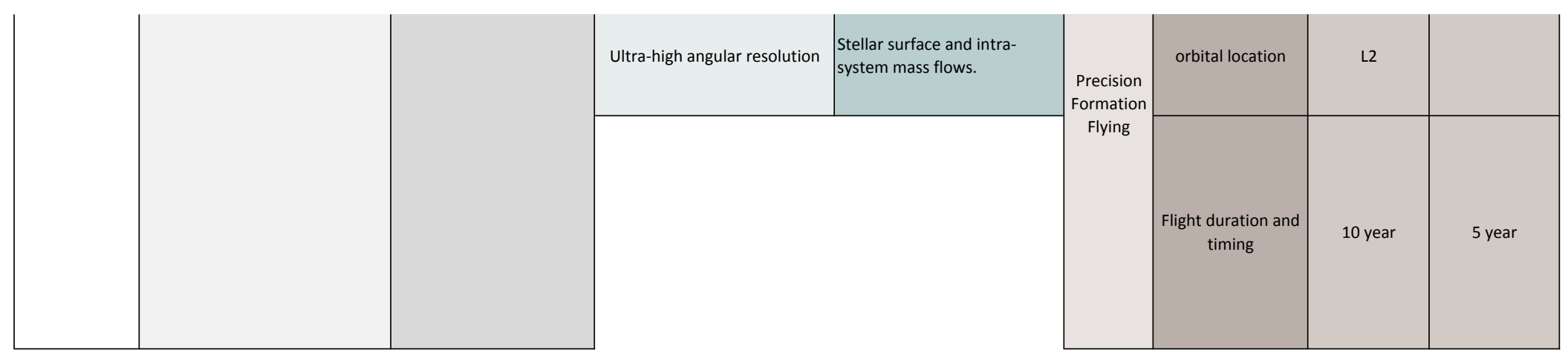

\begin{tabular}{|c|c|c|c|c|c|c|}
\hline & & & & & Sensitivity & $=$ Limiting Flux/SNR \\
\hline & & & & Intensity & $\begin{array}{l}\text { Dynamic } \\
\text { Range }\end{array}$ & $\begin{array}{l}=\text { Max Flux } / \\
\text { sensitivty }\end{array}$ \\
\hline & & & & Wavelength (Energy) & Bandwidth & 2.0 micronst \\
\hline & & & & 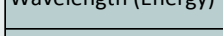 & Resolution & Broadband \\
\hline & $\begin{array}{l}\text { Systematic survey of } \\
\text { circumstellar environments to } \\
\text { determine distribution of matter. }\end{array}$ & $\begin{array}{l}\text { Detect spatial distribution } \\
\text { of dust, rings and } \\
\text { protoplanets. }\end{array}$ & $\begin{array}{l}\text { Extended circumstellar } \\
\text { emission. }\end{array}$ & Angular & \begin{tabular}{|l|} 
Resolution \\
Instantane \\
ous FOV \\
\end{tabular} & $\begin{array}{l}0.1^{\prime \prime} \\
60^{\prime \prime}\end{array}$ \\
\hline & & & & & $\begin{array}{l}\text { Pointing, } \\
\text { scanning, } \\
\text { etc }\end{array}$ & $0.01 "$ \\
\hline & & & & & $\begin{array}{l}\text { Integration } \\
\text { time }\end{array}$ & Background limited \\
\hline & & & & Temporal & \begin{tabular}{|l|} 
Single \\
observatio \\
n duration \\
\end{tabular} & Jitter limited \\
\hline & & & & Intensity & Sensitivity & $=$ Limiting Flux/SNR \\
\hline & & & & & \begin{tabular}{|l|} 
Dynamic \\
Range
\end{tabular} & $\begin{array}{l}=\text { Max Flux } \\
\text { sensitivty }\end{array}$ \\
\hline & & & & |Wavelength (Energy) & Bandwidth & $550 \mathrm{~nm}$ \\
\hline & Detect clouds and surface & Time resolved reflectivity & Exoplanet light curves. & & Resolution & \begin{tabular}{|l|} 
Broadband \\
$0.01 "$
\end{tabular} \\
\hline & features of exoplanets. & of exoplanets. & & Angular & \begin{tabular}{|l} 
Instantane \\
ous FOV
\end{tabular} & $10^{\prime \prime}$ \\
\hline $\begin{array}{l}\text { Conduct } \\
\text { observations }\end{array}$ & & & & Jilloulat & $\begin{array}{l}\text { Pointing, } \\
\text { scanning, } \\
\text { etc }\end{array}$ & $0.001 "$ \\
\hline $\begin{array}{l}\text { over UVOIR } \\
\text { wavelengths, }\end{array}$ & & & & & \begin{tabular}{|l|} 
Integration \\
time \\
\end{tabular} & Background limited \\
\hline $\begin{array}{l}\text { that contribute } \\
\text { to the } \\
\text { understanding }\end{array}$ & & & & Temporal & \begin{tabular}{|l|} 
Single \\
observatio \\
n duration
\end{tabular} & Jitter limited \\
\hline $\begin{array}{l}\text { of exoplanets } \\
\text { ons }\end{array}$ & & & & & Sensitivity & $=$ Limiting Flux/SNR \\
\hline $\begin{array}{l}\text { and the } \\
\text { circumstellar }\end{array}$ & & & & Intensity & \begin{tabular}{|l|} 
Dynamic \\
Range
\end{tabular} & $\begin{array}{l}=\text { Max Flux/ } \\
\text { sensitivty }\end{array}$ \\
\hline environment, & & & & Wavelength (Energy) & Bandwidth & 2.0 mcronst \\
\hline the low mass & & & & & \begin{tabular}{|l} 
Resolution \\
Resolution
\end{tabular} & \begin{tabular}{|l} 
Broadband \\
$1 "$
\end{tabular} \\
\hline $\begin{array}{l}\text { end of the } \\
\text { stellar IMF, the } \\
\text { hantrmaund }\end{array}$ & Constrain low end of the stellar & Deep imaging of star & & & \begin{tabular}{|l|} 
Instantane \\
ous FOV
\end{tabular} & $300 "$ \\
\hline
\end{tabular}

\section{1:1e9}

Roll control +/- 15

a

EO, GEO or L2

stable PS

All sky survey

1/10 Pointing better

than pixel size.

Operate for $5+$ years.

Maintain fine

guidance lock

between orientation

with muliple GS

acquisitions. 


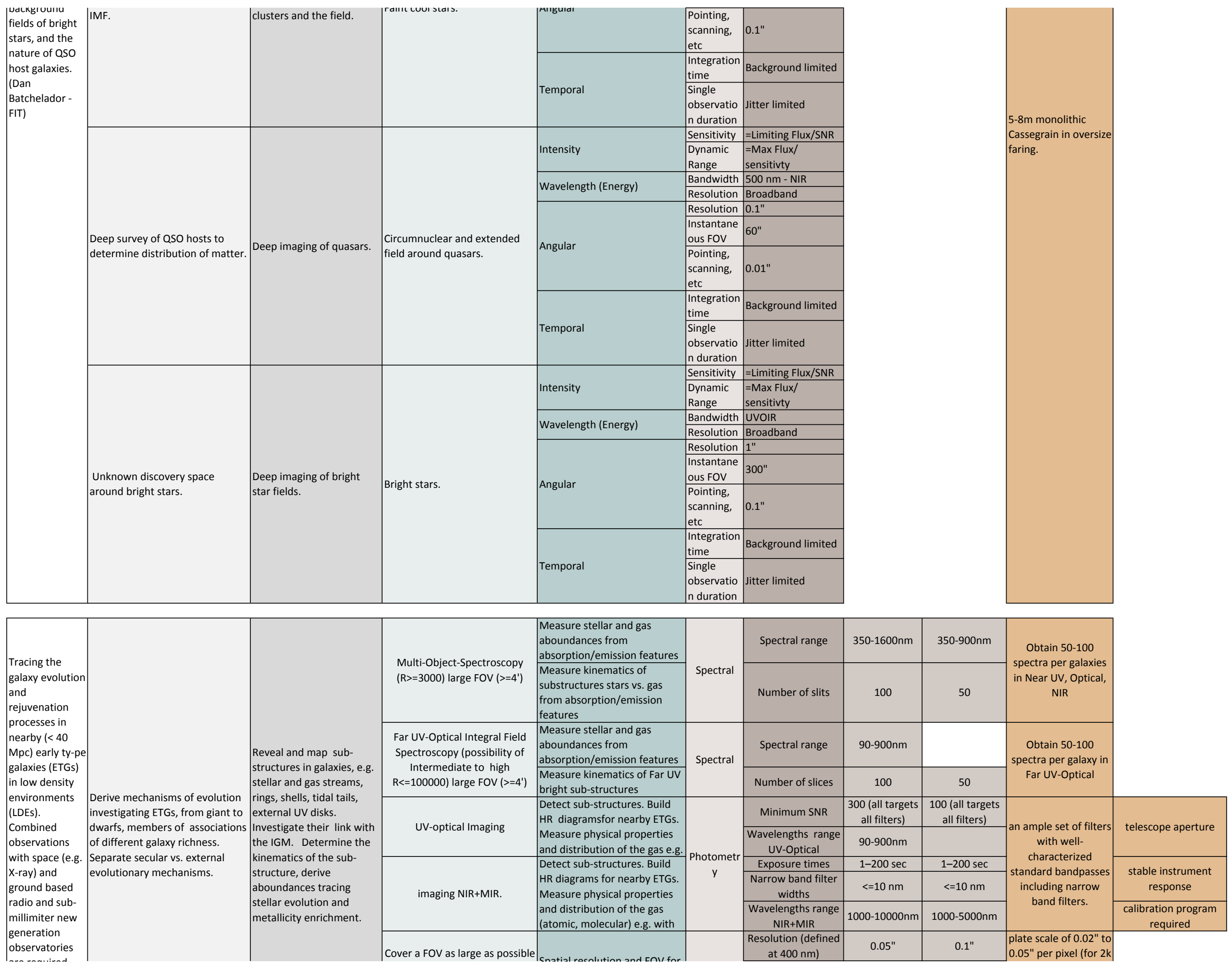








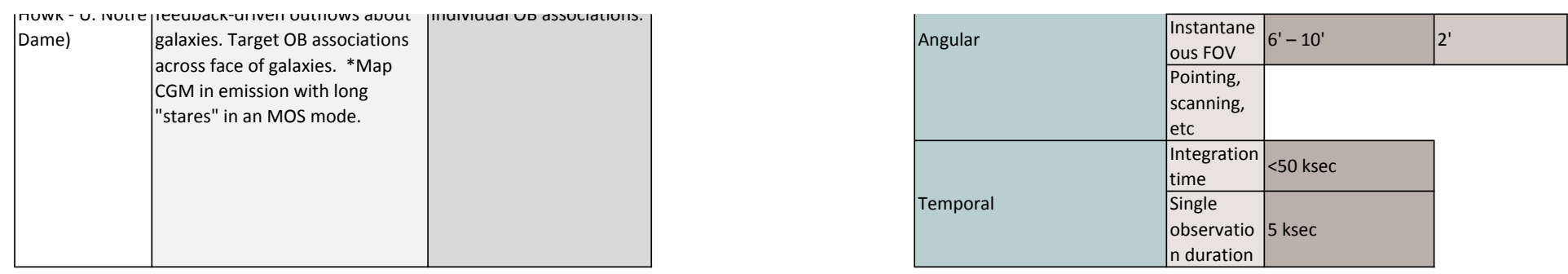

\begin{tabular}{|c|c|c|}
\hline $\begin{array}{l}\text { Conduct a } \\
\text { survey of the } \\
\text { baryonic and } \\
\text { metal content } \\
\text { in the } \\
\text { circumgalactic } \\
\text { medium (CGM) } \\
\text { of galaxies as a } \\
\text { function of gas } \\
\text { "phase." Trace } \\
\text { the exchange of } \\
\text { matter between } \\
\text { galaxies and the } \\
\text { intergalactic } \\
\text { medium. (Chris } \\
\text { Howk - U. Notre } \\
\text { Dame) }\end{array}$ & $\begin{array}{l}\text { *Map the circumgalactic medium } \\
\text { absorption about individual } \\
\text { galaxies toward several } \\
\text { background sources at high } \\
\text { resolution and signal-to-noise. } \\
{ }^{*} \text { Measure velocities of } \\
\text { circumgalactic gas with respect } \\
\text { to galaxy host. }{ }^{*} \text { Assess } \\
\text { metallicity from inner } \\
\text { circumgalactic medium through } \\
\text { the virial radius and into the } \\
\text { intergalactic medium. *Use } \\
\text { multiple ions to probe the } \\
\text { distribution of baryons, metals } \\
\text { with temperature. Probe the } \\
\text { hottest } 10^{\wedge} 6 \text { K gas at nearly the } \\
\text { virial temperature with FUV/EUV } \\
\text { transitions (O VI, Ne VIII, Mg X, Si } \\
\text { XII) ; probe the cooler gas with H } \\
\text { I, Si II, Si III, Si IV, C II, C III, C IV. }\end{array}$ & \begin{tabular}{|l} 
High resolution \\
spectroscopy of UV \\
wavelengths giving access \\
to redshifted EUV/UV \\
absorption/emission \\
diagnostics
\end{tabular} \\
\hline
\end{tabular}

\begin{tabular}{|c|c|c|c|c|}
\hline $\begin{array}{l}\text { Detect weak EUV transitions } \\
\text { tracing the hottest gas, low } \\
\text { metal column density tracing } \\
\text { IGM inflow. }\end{array}$ & Intensity & SNR & 50 & 25 \\
\hline $\begin{array}{l}\text { Observe H I features incl. } 1 \text { Ryd } \\
\text { break throughout Universe; } \\
\text { access critical EUV transitions. }\end{array}$ & \multirow[t]{2}{*}{ Wavelength } & Range & $900-3200 \AA$ & $1000-3200$ \\
\hline $\begin{array}{l}\text { Resolve internal motions in } \\
\text { CGM/diff. between phases. }\end{array}$ & & Resolution & $45,000-60,000$ & 20,000 \\
\hline & \multirow{3}{*}{ Angular } & Resolution & & \\
\hline & & $\begin{array}{l}\text { Instantane } \\
\text { ous FOV }\end{array}$ & & \\
\hline & & $\begin{array}{l}\text { Pointing, } \\
\text { scanning, } \\
\text { etc }\end{array}$ & & \\
\hline & \multirow[b]{2}{*}{ Temporal } & $\begin{array}{l}\text { Integration } \\
\text { time }\end{array}$ & $<50 \mathrm{ksec}$ & \\
\hline & & \begin{tabular}{|l|} 
Single \\
observatio \\
n duration \\
\end{tabular} & $5 \mathrm{ksec}$ & \\
\hline
\end{tabular}

High SNR needed for

weak metal lines.

Pushes to large

aperture, good

reflectivity.

Resolution+SNR push

large apertures.

Need for multiple

Need for multiple

background objects

(notably QSOs) also

to provide necessary

spectral resolution.

Key for CGM science

is stable, well-

characterized

spectral ISF,

preferrably very

Exposure times short

enough to do many

QSOs behind

individual galaxies

\begin{tabular}{|l|l|l|}
\hline & & \\
$\begin{array}{l}\text { Conduct } \\
\text { ultraviolet to } \\
\text { optical } \\
\text { observations, } \\
\text { that contribute } \\
\text { to the } \\
\text { understanding } \\
\text { of the evolution } \\
\text { and fates of } \\
\text { massive stars. } \\
\text { (Aida Wofford - } \\
\text { Institut } \\
\text { d'Astrophysique } \\
\text { de Paris) }\end{array}$ & $\begin{array}{l}\text { Collection of statistically } \\
\text { significant samples over the } \\
\text { that are extreme in terms of SFR, } \\
\text { metallicity, reddening. }\end{array}$ & $\begin{array}{l}\text { Derive statistics on the } \\
\text { physical properties of } \\
\text { massive stars, their } \\
\text { evolved descendants, and } \\
\text { the precursors of } \\
\text { supernovae (temperature, } \\
\text { luminosity, mass loss rate, } \\
\text { rotation speed, binary } \\
\text { masses and periods, } \\
\text { chemical composition of } \\
\text { atmospheres and ejecta, } \\
\text { spatial location as a } \\
\text { function of type, } \\
\text { variability) }\end{array}$ \\
\hline & & \\
& & \\
\end{tabular}

\begin{tabular}{|c|c|}
\hline \multirow{2}{*}{$\begin{array}{c}\text { Cover a range of wavelengths in } \\
\text { UV and visible }\end{array}$} & $\begin{array}{l}\text { Measure stellar temperature, } \\
\text { luminosity, mass, mass loss } \\
\text { rate, terminal velocity, } \\
\text { rotation speed }\end{array}$ \\
\hline & $\begin{array}{l}\text { Measure chemical } \\
\text { composition of atmospheres } \\
\text { and ejecta }\end{array}$ \\
\hline $\begin{array}{l}\text { Take high-quality images of } \\
\text { individual massive stars in }\end{array}$ & $\begin{array}{l}\text { Study spatial location of } \\
\text { massive stars and their }\end{array}$ \\
\hline \multirow[b]{2}{*}{$\begin{array}{c}\text { Cover a range of wavelengths in } \\
\text { UV and visible }\end{array}$} & $\begin{array}{l}\text { Measure extinction due to } \\
\text { dust towards many sightlines }\end{array}$ \\
\hline & \\
\hline $\begin{array}{l}\text { Sample at cadence tied to } \\
\text { binary orbital motions }\end{array}$ & $\begin{array}{l}\text { Obtain masses and periods of } \\
\text { binaries }\end{array}$ \\
\hline \multirow{2}{*}{$\begin{array}{l}\text { Sample at multiple epochs to } \\
\text { study history of mass loss }\end{array}$} & $\begin{array}{l}\text { Study eruptions of LBVs and } \\
\text { other massive star } \\
\text { descendants }\end{array}$ \\
\hline & $\begin{array}{l}\text { Study conditons prior to } \\
\text { supernova explosions }\end{array}$ \\
\hline
\end{tabular}

\begin{tabular}{c|c|}
$\begin{array}{c}\text { ??+ filter imaging in } \\
\text { UV/vis range }\end{array}$ & \\
\hline $\begin{array}{c}\text { instrument } \\
\text { throughput }\end{array}$ & telescope aperture \\
$\begin{array}{c}\text { ??+ filters with well- } \\
\text { characterized } \\
\text { bandpasses }\end{array}$ & \\
\hline $\begin{array}{c}\text { stable instrument } \\
\text { response }\end{array}$ & $\begin{array}{c}\text { calibration program } \\
\text { requirements }\end{array}$ \\
\hline
\end{tabular}

\begin{tabular}{|c|c|c|c|c|c|}
\hline & & & & \multirow{2}{*}{ UV/vis range } & \\
\hline & Spectral resolution & - & 20,000 & & \\
\hline \multirow{5}{*}{$\begin{array}{l}\text { tometr } \\
y\end{array}$} & Minimum SNR & - & 25 & \multirow{2}{*}{$\begin{array}{l}\text { instrument } \\
\text { throughput }\end{array}$} & \multirow{2}{*}{ telescope aperture } \\
\hline & Exposure times & - & - & & \\
\hline & Central wavelengths & $90-1000 \mathrm{~nm}$ & $120-1000 \mathrm{~nm}$ & \multirow{2}{*}{$\begin{array}{c}\text { ??+ filters with well- } \\
\text { characterized } \\
\text { bandpasses }\end{array}$} & \\
\hline & $\begin{array}{c}\text { Accuracy and } \\
\text { systematic error }\end{array}$ & - & - & & \\
\hline & \begin{tabular}{|c}
$\begin{array}{c}\text { Resolution (defined } \\
\text { at } 150 \mathrm{~nm} \text { ) }\end{array}$ \\
\end{tabular} & $0.05 "$ & $0.1^{\prime \prime}$ & $\begin{array}{c}\text { stable instrument } \\
\text { response }\end{array}$ & $\begin{array}{c}\text { calibration program } \\
\text { requirements }\end{array}$ \\
\hline
\end{tabular}

\begin{tabular}{c|c|c|c}
$\begin{array}{c}\text { Stable distortion } \\
\text { solution }\end{array}$ & $\begin{array}{c}0.1 \text { " error over } \\
\text { FOV }\end{array}$ & $\begin{array}{c}0.1 \text { " error over } \\
\text { FOV }\end{array}$ & $\begin{array}{c}\text { plate scale of } 0.02 " \text { to } \\
0.05 " \text { per pixel (for } 2 \mathrm{k}\end{array}$ \\
\cline { 2 - 3 } & & &
\end{tabular}

$\times 2 \mathrm{k}$ array); stable

telescope assembly

Acquire and

readout time

?? $\mathrm{min}$

readout time $<$ ?? 


\begin{tabular}{|c|c|c|c|c|c|c|}
\hline \multirow{15}{*}{\begin{tabular}{|l|} 
Conduct \\
observations \\
from the UV to \\
the radio to \\
understand star \\
formation \\
within galaxies \\
and how it \\
drives galaxy \\
growth \\
througout space \\
and time. \\
(Daniela Calzetti \\
- U. \\
Massachusetts)
\end{tabular}} & \multirow{15}{*}{$\begin{array}{l}\text { Observe nearby galaxies within } \\
100 \text { Mpc to obtain statistics } \\
\text { within a representative volume } \\
\text { of the Universe of star formation } \\
\text { histories with lookback time from } \\
\sim 1 \text { Myr to } 13 \text { Gyr. } \\
\\
\end{array}$} & \multirow{5}{*}{$\begin{array}{l}\text { Determine the Upper End } \\
\text { (slope, maximum star } \\
\text { mass) of the Stellar Initial } \\
\text { Mass Function }\end{array}$} & $\begin{array}{l}\text { Isolate massive stars within star } \\
\text { clusters out to } 10 \mathrm{Mpc} \text { and } \\
\text { individual star clusters out to } \\
100 \mathrm{Mpc}\end{array}$ & Intensity & \begin{tabular}{|l} 
Dynamic \\
Range
\end{tabular} & $\begin{array}{l}2 \mathrm{M} \text { M_sun star at } 10 \\
\text { Mpc in } 0.1 \text { micron } \\
\text { band } \\
2-300 \mathrm{M} \text { _sun stars } \\
\text { at } 10 \mathrm{Mpc}\end{array}$ \\
\hline & & & $\begin{array}{l}\text { by getting their UV-to-nearlR } \\
\text { spectra plus }\end{array}$ & Wavelength (Energy) & \begin{tabular}{|l|} 
Bandwidth \\
\end{tabular} & 0.09-1.2 micron \\
\hline & & & $\begin{array}{l}\text { with spatial resolution of } 0.05 \\
\mathrm{pc} \text { (per pixel) at } 5 \mathrm{Mpc} \text { and } 1 \mathrm{pc} \\
\text { at } 100 \mathrm{Mpc}\end{array}$ & \multirow[b]{2}{*}{ Angular } & Resolution & 0.004" (2 pixels) \\
\hline & & & $\begin{array}{l}\text { and spatial coverage of } 3 \\
\text { arcmin } \times 3 \text { arcmin (rough galaxy } \\
\text { size) }\end{array}$ & & \begin{tabular}{|l|} 
Instantane \\
ous FOV \\
Pointing, \\
scanning, \\
etc \\
\end{tabular} & $3^{\prime} \times 3^{\prime}$ \\
\hline & & & $\begin{array}{l}\text { Simultaneous coverage of } \\
\text { multiple stars and star clusters } \\
\text { (multi-object spectroscopy) }\end{array}$ & Angular & \begin{tabular}{|l|}
$\begin{array}{l}\text { Aperture } \\
\text { size } \\
\text { (spectrosc } \\
\text { opy) } \\
\text { number of } \\
\text { apertures }\end{array}$ \\
\end{tabular} & $\begin{array}{l}>10000 \text { each } \\
\text { pointing }\end{array}$ \\
\hline & & \multirow{5}{*}{$\begin{array}{l}\text { Reconcile the Local and } \\
\text { Cosmic Star Formation } \\
\text { Histories }\end{array}$} & \multirow{3}{*}{\begin{tabular}{|l|} 
Recent-past star formation \\
histories (past 1 Gyr with 10 \\
Myr time resolution) of local \\
by getting their UV-to-nearlR \\
broad/medium/narrow band \\
with spatial resolution of 0.05 \\
pc (per pixel) at $5 \mathrm{Mpc}$ \\
\end{tabular}} & Intensity & \begin{tabular}{|l|} 
Sensitivity \\
Dynamic \\
Range \\
\end{tabular} & m_UV 31 mag \\
\hline & & & & Wavelength (Energy) & \begin{tabular}{|l|} 
Bandwidth \\
Resolution \\
\end{tabular} & \begin{tabular}{|l|}
$0.15-1.5$ micron \\
$10-300$
\end{tabular} \\
\hline & & & & & Resolution & $0.004 "$ (2 pixels) \\
\hline & & & and spatial coverage of 3 & & $\begin{array}{l}\text { Instantane } \\
\text { ous FOV }\end{array}$ & $3^{\prime} \times 3^{\prime}$ \\
\hline & & & $\begin{array}{l}\operatorname{arcmin} \times 3 \operatorname{arcmin} \text { (rough galaxy } \\
\text { size) }\end{array}$ & & \begin{tabular}{|l} 
Pointing, \\
scanning, \\
etc
\end{tabular} & \\
\hline & & \multirow{5}{*}{$\begin{array}{l}\text { Determine the growth of } \\
\text { galaxies by studying the } \\
\text { stellar population content, } \\
\text { star formation modes, and } \\
\text { star formation histories of } \\
\text { their outskirts }\end{array}$} & \multirow{3}{*}{$\begin{array}{l}\text { Color-magnitude diagrams of } \\
\text { stars down to } 0.5-1 \text { mag below } \\
\text { the Main Sequence turn off at } \\
\text { UV-to-nearlR medium/broad } \\
\text { band photometry }\end{array}$} & \multirow[b]{2}{*}{ Intensity } & Sensitivity & $\mathrm{m} \_\mathrm{V}=35 \mathrm{mag}$ \\
\hline & & & & & $\begin{array}{l}\text { Dynamic } \\
\text { Range }\end{array}$ & 8-10 mag \\
\hline & & & & Wavelength (Energy) & \begin{tabular}{|l|} 
Bandwidth \\
Resolution
\end{tabular} & \begin{tabular}{|l|}
$0.15-1.5$ micron \\
$10--50$
\end{tabular} \\
\hline & & & $\begin{array}{l}\text { with spatial resolution of } 0.1 \mathrm{pc} \\
\text { (per pixel) at } 10 \mathrm{Mpc}\end{array}$ & \multirow[b]{2}{*}{ Angular } & Resolution & $0.004 "$ ( 2 pixels) \\
\hline & & & $\begin{array}{l}\text { and spatial coverage of } 3 \\
\text { arcmin } \times 3 \text { arcmin (rough galaxy } \\
\text { size) }\end{array}$ & & \begin{tabular}{|l|} 
Instantane \\
ous FOV \\
Pointing, \\
scanning, \\
etc \\
\end{tabular} & $3^{\prime} \times 3^{\prime}$ \\
\hline \multirow{5}{*}{\begin{tabular}{|l|} 
Understand \\
how \\
protoplanetary \\
disks evolve and \\
form planetary \\
systems (Kevin \\
France - \\
U.Colorado)
\end{tabular}} & \multirow{5}{*}{$\begin{array}{l}\text { Determine the accretion } \\
\text { luminosity of protostars, the } \\
\text { composition of the inner } 10 \mathrm{AU} \\
\text { of planet-forming disks, and the } \\
\text { lifetime of gas disks in young } \\
\text { planetary systems }\end{array}$} & \multirow{5}{*}{$\begin{array}{l}\text { Mass accretion rates of } \\
\text { protostars; abundances, } \\
\text { physical conditions, and } \\
\text { lifetimes of molecular gas } \\
\text { in the inner regions of } \\
\text { protoplanetary disks }\end{array}$} & \multirow{3}{*}{\begin{tabular}{|l|} 
1) Flux calibrated and spectrally \\
resolved ultraviolet/optical \\
spectra of $>30$ protostellar \\
systems in each of 10 star- \\
forming regions with a variety \\
2) Spectrally resolved \\
abosrption lines in high- \\
inclination disks: $\mathrm{H} 2, \mathrm{CO}, \mathrm{H} 2 \mathrm{O}$, \\
atomic species \\
\end{tabular}} & \multirow{2}{*}{ Intensity } & Sensitivity & $\begin{array}{l}\mathrm{S} / \mathrm{N}=10 @ 1 \mathrm{E}-16 \\
{[\mathrm{erg} / \mathrm{cm} 2 / \mathrm{s}] \text { per } 60 \mathrm{~s}}\end{array}$ \\
\hline & & & & & \begin{tabular}{|l|} 
Dynamic \\
Range
\end{tabular} & $\begin{array}{l}1 \mathrm{E}-11-1 \mathrm{E}-19 \\
{[\mathrm{erg} / \mathrm{cm} 2 / \mathrm{s} / \mathrm{A}]}\end{array}$ \\
\hline & & & & \multirow[b]{2}{*}{ Wavelength (Energy) } & Bandwidth & $\begin{array}{l}\text { 2a) } 91-170 \mathrm{~nm}, 2 \mathrm{~b}) \\
100-400 \mathrm{~nm}\end{array}$ \\
\hline & & & $\begin{array}{l}\text { 2b) } \mathrm{H} 2 \text { and } \mathrm{CO} \text { fluorescent } \\
\text { emission in disks of any } \\
\text { orientation }\end{array}$ & & Resolution & $\begin{array}{l}\text { 2a) } 3 \mathrm{~km} / \mathrm{s} \text { for lines } \\
\text { absorption lines, } 2 \mathrm{~b} \text { ) } \\
100 \mathrm{~km} / \mathrm{s} \text { for } \\
\text { emission lines }\end{array}$ \\
\hline & & & & & Resolution & $2^{\prime \prime}$ \\
\hline
\end{tabular}

In space, high QE detectors

high count rate detectors

optical coatings dow to $91 \mathrm{~nm}$. highresolutuion, lowscatter gratings. one high-resolution spectrograph and spectrograph and one multi-object spectrograph. flux standards for $2 \%$ absolute spectrophotometry 


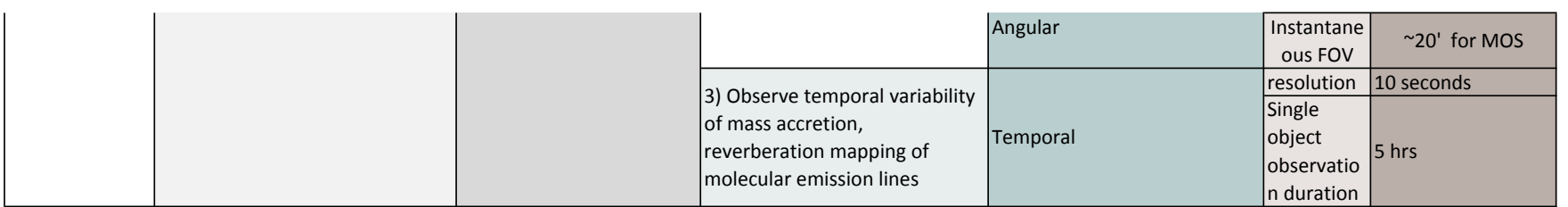

\begin{tabular}{|c|c|c|c|c|c|c|}
\hline \multirow{7}{*}{$\begin{array}{l}\text { Characterize } \\
\text { nearby } \\
\text { habitable } \\
\text { exoplanets } \\
\text { (Kevin France - } \\
\text { U. Colorado) }\end{array}$} & \multirow{7}{*}{$\begin{array}{l}\text { Determine the absolute level, the } \\
\text { spectral energy distrubution, and } \\
\text { the temporal variability of the } \\
\text { energetic radiation environment } \\
\text { around exoplanets to determine } \\
\text { atmospheric photochemistry on } \\
\text { habitable exoplanets and control } \\
\text { for biosignature false positives }\end{array}$} & \multirow{7}{*}{$\begin{array}{l}\text { Chromospheric, transition } \\
\text { region, and coronal } \\
\text { luminosity and activity } \\
\text { level of low-mass stars (G, } \\
\mathrm{K} \text {, and } \mathrm{M} \text { ) }\end{array}$} & $\begin{array}{l}\text { 1) Broadband spectrally } \\
\text { resolved ultraviolet irradiance } \\
\text { spectra of all habitable planet } \\
\text { candidates, N = TBD }\end{array}$ & Intensity & \begin{tabular}{|l} 
Dynamic \\
Range \\
\end{tabular} & \begin{tabular}{|l|}
$1 \mathrm{E}-11-1 \mathrm{E}-19$ [erg \\
$/ \mathrm{cm} 2 / \mathrm{s} / \mathrm{A}]$
\end{tabular} \\
\hline & & & \begin{tabular}{|l|} 
2) Absolute fluxes of spectrally \\
and temporally resolved upper \\
atmosphere emission lines: \\
\end{tabular} & \multirow[t]{2}{*}{ Wavelength (Energy) } & Bandwidth & $95-400 \mathrm{~nm}$ \\
\hline & & & \begin{tabular}{|rr|}
\multicolumn{3}{|c|}{ C III, O VI, LyA, O I, C II, Si } \\
IV, & C IV, He II, Fe \\
II, Mg II, Ca II & \\
\end{tabular} & & Resolution & $\begin{array}{l}15 \mathrm{~km} / \mathrm{s} \text { for lines, } \\
100 \mathrm{~km} / \mathrm{s} \\
\text { broadband }\end{array}$ \\
\hline & & & 3) ang resolved stellar LyA from & & Resolution & $0.2 \operatorname{arcsec}$ at LyA \\
\hline & & & \begin{tabular}{|l|} 
background (geo, \\
interplanetary)
\end{tabular} & Angular & \begin{tabular}{|c|} 
Instantane \\
ous FOV
\end{tabular} & $\begin{array}{c}>2 \operatorname{arcsec} \text { (no hard } \\
\text { requirement) }\end{array}$ \\
\hline & & & & & resolution & $1 \mathrm{sec}$ \\
\hline & & & $\begin{array}{l}\text { 4) Temporal variability of high- } \\
\text { energy emission lines on typical } \\
\text { timescales of UV flares }\end{array}$ & Temporal & \begin{tabular}{|l|} 
Single \\
object \\
observatio \\
n duration
\end{tabular} & 8 hrs \\
\hline
\end{tabular}

\begin{tabular}{|c|c|c|c|c|c|c|}
\hline \multirow{7}{*}{$\begin{array}{l}\text { Understand the } \\
\text { processes that } \\
\text { determine the } \\
\text { structure and } \\
\text { evolution of } \\
\text { planetary } \\
\text { atmospheres } \\
\text { (Kevin France - } \\
\text { U. Colorado) }\end{array}$} & \multirow{7}{*}{$\begin{array}{l}\text { Determine the heating rates, } \\
\text { mass-loss rates, compositions, } \\
\text { and thermodynamic structures of } \\
\text { the atmospheres of extrasolar } \\
\text { planets }\end{array}$} & \multirow{7}{*}{$\begin{array}{l}\text { Atmospheric mass-loss } \\
\text { rates from short-period } \\
\text { planets of multiple } \\
\text { atmospheric constituents. } \\
\text { The incident stellar high } \\
\text { energy radiation spectrum. }\end{array}$} & $\begin{array}{l}\text { 1) Spectrally resolved far-UV } \\
\text { transit observations of }>30 \\
\text { Jupiter-mass planets, }>20 \\
\text { Neptune-mass planets, and }> \\
10 \text { rocky planets. }>=3 \text { transits } \\
\end{array}$ & Intensity & \begin{tabular}{|l} 
Dynamic \\
Range \\
\end{tabular} & $\begin{array}{l}\mathrm{S} / \mathrm{N}=50 @ 1 \mathrm{E}-15 \\
{[\mathrm{erg} / \mathrm{cm} 2 / \mathrm{s}] \text { per } 60 \mathrm{~s}} \\
1 \mathrm{E}-11-1 \mathrm{E}-18[\mathrm{erg} \\
/ \mathrm{cm} 2 / \mathrm{s} / \mathrm{A}] \\
\end{array}$ \\
\hline & & & $\begin{array}{l}\text { 2) Transit depth as a function of } \\
\text { wavelength and orbital phase } \\
\text { for key atmospheric tracers: }\end{array}$ & \multirow[t]{2}{*}{ Wavelength (Energy) } & Bandwidth & $100-300 \mathrm{~nm}$ \\
\hline & & & $\begin{array}{l}\text { LyA, O I, C II, Mg II, H2 } \\
\text { (superposed on O VI, C II, N II, } \\
\text { and C III profiles) } \\
\end{array}$ & & Resolution & $3 \mathrm{~km} / \mathrm{s}$ \\
\hline & & & 3) ang resolved stellar LyA from & & Resolution & $0.2 \operatorname{arcsec}$ at LyA \\
\hline & & & \begin{tabular}{|l} 
background (geo, \\
interplanetary)
\end{tabular} & Angular & $\begin{array}{l}\text { Instantane } \\
\text { ous FOV }\end{array}$ & $\begin{array}{c}>2 \operatorname{arcsec} \text { (no hard } \\
\text { requirement) }\end{array}$ \\
\hline & & & & & resolution & $1 \mathrm{~min}$ \\
\hline & & & $\begin{array}{l}\text { 4) Observe pre-ingress, transt, } \\
\text { and post-egress stellar flux }\end{array}$ & Temporal & $\begin{array}{l}\text { Single } \\
\text { object } \\
\text { observatio } \\
\text { n duration }\end{array}$ & $8 \mathrm{hrs}$ \\
\hline
\end{tabular}

\begin{tabular}{|c|c|c|c|c|c|c|}
\hline \multirow{5}{*}{$\begin{array}{l}\text { Understand the } \\
\text { nature of stellar } \\
\text { winds, magnetic } \\
\text { fields, and } \\
\text { circumstellar } \\
\text { material in } \\
\text { massive evolved } \\
\text { stars and their } \\
\text { influences on } \\
\text { single and }\end{array}$} & \multirow{5}{*}{$\begin{array}{l}\text { Use UVV time-domain broadband } \\
\text { polarimetric and } \\
\text { spectropolarimetric observations } \\
\text { to characterize changes with time } \\
\text { (and orbital phase, for binaries) } \\
\text { of CIRs, magnetic field lines, } \\
\text { disks, and other stellar wind and }\end{array}$} & \multirow{5}{*}{$\begin{array}{l}\text { Shapes, sizes, extents, } \\
\text { temperatures, densities, } \\
\text { and compositions of } \\
\text { electron- and resonance- } \\
\text { line scattering regions in } \\
\text { the atmospheres, winds, }\end{array}$} & \multirow{2}{*}{$\begin{array}{l}\text { Orientation of primary system } \\
\text { axis (e.g., binary orbital plane, } \\
\text { elongation of SN ejecta) }\end{array}$} & \multirow{2}{*}{$\begin{array}{l}\text { Broadband linear polarimetry } \\
\text { (Stokes } \mathrm{I}, \mathrm{Q}, \mathrm{U})\end{array}$} & Sensitivity & $\begin{array}{l}\mathrm{S} / \mathrm{N} \text { in total light }= \\
100 \text { in } 30 \mathrm{~min}\end{array}$ \\
\hline & & & & & \begin{tabular}{|l} 
Dynamic \\
Range
\end{tabular} & $\begin{array}{l}P=0-10 \% \text { with } \mathrm{s}_{\mathrm{P}} / \mathrm{P} \\
<0.1\end{array}$ \\
\hline & & & \multirow{2}{*}{$\begin{array}{l}\text { Gas distribution, clumpiness, } \\
\text { composition, temperature, } \\
\text { density, ionization state; } \\
\text { magnetic field strength and } \\
\text { geometry via Hanle effect }\end{array}$} & \multirow[t]{2}{*}{$\begin{array}{l}\text { Linear spectropolarimetry } \\
(\text { Stokes } I, Q, U)\end{array}$} & Bandwidth & $\begin{array}{l}\text { 100-900 } \mathrm{nm} \text { for key } \\
\text { diagnostic lines } \\
\text { (Lya, Ha, UV wind } \\
\text { lines) }\end{array}$ \\
\hline & & & & & Resolution & $\begin{array}{l}\mathrm{R}=25,000 \text { (UV) to } \\
35,000 \text { (visible) }\end{array}$ \\
\hline & & & & & Bandwidth & $\begin{array}{l}\text { 100-900 } \mathrm{nm} \text { for key } \\
\text { diagnostic lines } \\
\text { (Lya, Ha, UV wind } \\
\text { lines) }\end{array}$ \\
\hline
\end{tabular}

In space, high QE

detectors

high count rate

detectors

optical coatings dow

to $91 \mathrm{~nm}$. gratings.

lux standards for $2 \%$

absolute

spectrophotometry

$\mathrm{N} / \mathrm{a}$

$\mathrm{N} / \mathrm{a}$

photon-counting

detectors, L2 or

elliptical orbit

In space, high QE

detectors

high count rate

detectors

optical coatings dow

to $100 \mathrm{~nm}$. high-

resolutuion, low-

scatter gratings

N/a

N/a

photon-counting

detectors, L2 or

elliptical orbit

Instrumental

polarization < $3 \%$.

Polarized and

unpolarized standard

Instrumental

polarization $<3 \%$.

Polarized and

unpolarized standard

stars.

Good pointing

stability $(\sim 0.1 \mathrm{~km} / \mathrm{s}$

between spectra in a 
binary stellar CSM structures; as well as evolution and illuminating the characteristics SN/GRB

progenito

progenitor

pathways.

Hoffman - U.

Denver) $\mathrm{SN}$ ejecta and surroundings that history. and binary stars and

feld geome 3-D magnetic field geometries in single and binary evolved stars.

.

\begin{tabular}{|c|c|c|c|}
\hline geometry via Zeeman effect & (Stokes V) & Resolution & $\begin{array}{l}\mathrm{R}=25,000 \text { (UV) to } \\
35,000 \text { (visible) }\end{array}$ \\
\hline & & Resolution & \\
\hline & Angular & \begin{tabular}{|l|} 
Instantane \\
ous FOV
\end{tabular} & \\
\hline \multirow[b]{2}{*}{$\begin{array}{l}\text { Periodicities including rotation, } \\
\text { orbital motion, and CIR } \\
\text { modulations; time evolution of } \\
\text { SN ejecta and CSM }\end{array}$} & & Resolution & $30 \mathrm{~min}$ \\
\hline & Temporal & \begin{tabular}{|l|} 
Single \\
object \\
observatio \\
n duration
\end{tabular} & $\begin{array}{l}\text { few days for entire } \\
\text { rotation period }\end{array}$ \\
\hline
\end{tabular}

Derive statistics of the disk

dispersal time scale as a

function of the stellar and

environmental properties,

including stars with

different spectral types

and ages, and in stellar

clusters with different sta

formation histories.

Compare the dispersal of

proto-planetary disks in

low-mass star forming

intermediate mass star

forming regions such as

Orion, and in high mass

as Carina.
and

\section{Spectratrange}

as velocity structure in the

photoevaporating wind

\section{Investigate the}

dispersal Use narrow-band imaging and

machanism of high resolution spectroscopy to

proto-planetary map the morphology and

disks through kinematics of photoevaporating

\begin{tabular}{c|c}
$\begin{array}{c}\text { observations of } \\
\text { gas forbidden }\end{array}$ & $\begin{array}{c}\text { winds and invesigate the time } \\
\text { scale for the disk dispersal in }\end{array}$ \\
lines (Patick & sstems tat are
\end{tabular}

lines (Patrick systems that are in the process of

lines (Patrick
Hartigan - Rice $\begin{gathered}\text { systems that are in the process } \\ \text { forming planets }\end{gathered}$

U.)

regions such as Taurus and

Chamaleon, with that in

A range of emission lines in
near UV, visible, and near IR,
including [O I] 6300A, Ha, and
$\mathrm{Mg} I I 2800 \mathrm{~A}$

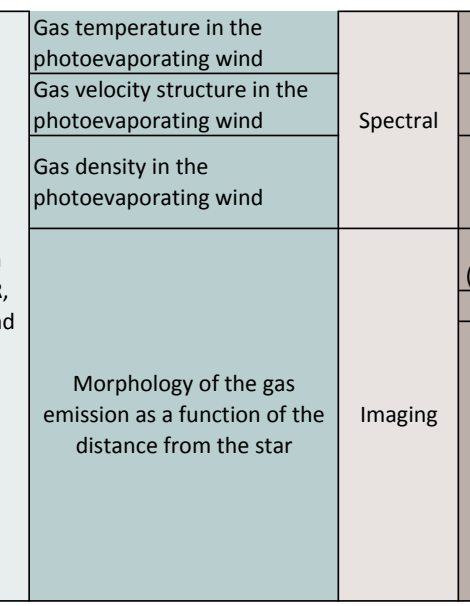

\begin{tabular}{|c|c|c|c|c|}
\hline Spectral range & $1200 \mathrm{~A}-9000 \mathrm{~A}$ & $2700 \mathrm{~A}-7000 \mathrm{~A}$ & \multirow{3}{*}{$\begin{array}{l}\text { Spectrograph with } \\
\text { Iong slit capability. An } \\
\text { IFU system would be } \\
\text { great if no spatial } \\
\text { resolution is lost }\end{array}$} & \multirow{3}{*}{$\begin{array}{c}\text { Possible synergy with } \\
\text { needs of extragalactic } \\
\text { community to spectro- } \\
\text { image multiple sources } \\
\text { in a field }\end{array}$} \\
\hline Spectral resolution & $R$ up to 30,000 & $\mathrm{R}$ up to 10,000 & & \\
\hline Spatial resolution & $\begin{array}{c}20 \text { arcsecond } \\
\text { slits @ } \lambda / D \\
\text { pixel scale }\end{array}$ & $\begin{array}{c}5 \text { arcsecond slits } \\
@ \lambda / D \text { pixel } \\
\text { scale }\end{array}$ & & \\
\hline $\begin{array}{l}\text { Spatial Resolution } \\
\text { (defined at } 400 \mathrm{~nm} \text { ) }\end{array}$ & $\lambda / D$ pixel scale & $\lambda / D$ pixel scale & & \multirow[b]{3}{*}{$\begin{array}{l}12 \mathrm{~m} \text { primary to give } \\
\text { spatial resolution, flux } \\
\text { sensitivity, and Strehl } \\
\text { ratio needed to image } \\
\text { faint extended } \\
\text { structures reliably } \\
\text { around bright point } \\
\text { sources }\end{array}$} \\
\hline FOV & 5 arcminutes & 3 arcminutes & & \\
\hline Filters & $\begin{array}{c}16 \text { narrowband } \\
\text { nebular filters, } \\
\text { among them CI } \\
9850, \mathrm{NII} 6583, \\
\mathrm{NI} 5200, \mathrm{OI} \\
6300, \mathrm{OII} 3727, \\
\text { SII 6716, SII } \\
6731, \mathrm{Mg} \mathrm{II} \\
2800, \mathrm{H \alpha}\end{array}$ & $\begin{array}{l}12 \text { filters } \\
\text { minimum }\end{array}$ & $\begin{array}{c}\text { Imager with excellent } \\
\text { filter options and a } \\
\text { modest FOV }\end{array}$ & \\
\hline
\end{tabular}

Count and measure the Individual stars in young \begin{tabular}{l|l} 
physical parameters to & clusters down \\
at $5 \mathrm{Mpc}$.
\end{tabular}

What controls Observationally constrain models $10 \%$ accuracy of individual the mass- $\quad$ of the formation and evolution of stars down to 5 solar energy- $\quad$ the massive stars that drive metal masses in star clusters out \begin{tabular}{l|l} 
chemical cycles production and distribution & to $5 \mathrm{Mpc}$ (e.g.,
\end{tabular}

within galaxies? through libraries of resolved NGC253,M82), covering

(Ben Williams - massive stars in a wide range of metallicities from 0.1-2.0

U. Washington) formation environments.

solar and galaxy masses

ranging from $10^{\wedge} 5-10^{\wedge} 12$

solar masses

\begin{tabular}{|l|}
\hline Angular Resolution \\
\hline Field of View \\
\hline Number of Filters \\
\hline Wavelength Coverage \\
\hline
\end{tabular}

Brightnesses of such individual

stars in at least 6 independen

bands covering $0.2-1.0 \mathrm{micr}$

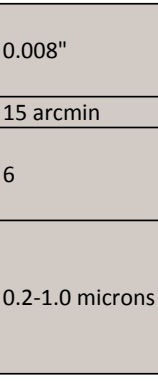

line combination.

Simultaneous

wavelength

calibration.

Monitoring capability to characterize

orbital periods up to

timescales of months

\begin{tabular}{|c|c|c|c|c|c|c|c|c|c|c|}
\hline \multirow{6}{*}{$\begin{array}{l}\text { Measure } \\
\text { magnetic field } \\
\text { and dust } \\
\text { characteristics } \\
\text { in the } \\
\text { interstellar } \\
\text { medium (BG } \\
\text { Andersson - } \\
\text { NASA Ames) }\end{array}$} & \multirow{6}{*}{$\begin{array}{l}\text { Use spectropolarimetry - over } \\
\text { the UV (and optical) range - to } \\
\text { determine the amount and } \\
\text { orientation of the dichroic } \\
\text { extinction polarization of } \\
\text { background stars due to aligned } \\
\text { dust. }\end{array}$} & \multirow{6}{*}{$\begin{array}{l}\text { 1) Measure the magnetic } \\
\text { field strength in diffuse gas } \\
\text { and 2) characterize the } \\
\text { dust properties by } \\
\text { analyzingth the } \\
\text { polarization spectra in the } \\
\text { context of modern grain } \\
\text { alignment theory }\end{array}$} & $\begin{array}{c}\text { Medium to high-spectral } \\
\text { resolution spectropolarimetry } \\
\text { in the UV }\end{array}$ & $\begin{array}{l}\text { Size distribution alignment } \\
\text { fraction and mineralogy of the } \\
\text { small dust }\end{array}$ & Spectral & Resolving power & $160-1600 \mathrm{~nm}$ & $300-500 \mathrm{~nm}$ & $\begin{array}{c}\text { Instrumental } \\
\text { polarization, stability } \\
\text { and variations over } \\
\text { the FOV }\end{array}$ & $\begin{array}{c}\text { Sensitivity to assemble } \\
\text { statistically significant } \\
\text { samples of polarimetry } \\
\text { of background stars }\end{array}$ \\
\hline & & & $\begin{array}{l}\text { Spectropolarimetry of the } \\
2175 \AA \text { extinction feature }\end{array}$ & $\begin{array}{l}\text { Statistics of polarization of } \\
\text { feature }\end{array}$ & \multirow[b]{2}{*}{ Spectral } & Spectral range & $210-130 \mathrm{~nm}$ & & \multirow{2}{*}{$\begin{array}{l}\text { Sensitivity, } \\
\text { instrumental } \\
\text { polarization }\end{array}$} & \\
\hline & & & & $\begin{array}{l}\text { Establish carrier and improve } \\
\text { it's use in extinction curves } \\
\text { etc. }\end{array}$ & & Resolving power & 1000 & 200 & & \\
\hline & & & \multirow[t]{2}{*}{$\begin{array}{l}\text { Tracing of the polarization } \\
\text { curve to FUV wavelengths }\end{array}$} & \multirow{2}{*}{$\begin{array}{l}\text { Test ans utilize the theoretical } \\
\text { prediction that paramagnetic } \\
\text { alignment dominates for the } \\
\text { very smallest grains, in which }\end{array}$} & \multirow{2}{*}{$\begin{array}{c}\text { Photometr } \\
y\end{array}$} & $\begin{array}{l}\text { Sensitivity and } \\
\text { systematic error }\end{array}$ & $0.10 \%$ & & \multirow{2}{*}{$\begin{array}{l}\text { Sensitivity, } \\
\text { instrumental } \\
\text { polarization }\end{array}$} & \\
\hline & & & & & & number of fileters & $\begin{array}{c}4 \text { for } 120- \\
250 \mathrm{~nm}\end{array}$ & 2 for $120-250 \mathrm{~nm}$ & & \\
\hline & & & $\begin{array}{l}\text { Line polarimetry of fine } \\
\text { structure lines }\end{array}$ & $\begin{array}{l}\text { Measure magnetic field } \\
\text { strengths through the Hanle }\end{array}$ & Spectral & $\begin{array}{c}\text { Spectral range } \\
\text { Spectral resolution }\end{array}$ & $\begin{array}{c}120-1600 \mathrm{~nm} \\
50,000\end{array}$ & 5,000 & $\begin{array}{l}\text { instrument } \\
\text { throughput }\end{array}$ & telescope aperture \\
\hline
\end{tabular}

